University of Louisville

ThinkIR: The University of Louisville's Institutional Repository

Electronic Theses and Dissertations

$12-2015$

\title{
The impact of an interactive technology application on elementary student achievement in fractions.
}

Shannon M. Stone

University of Louisville

Follow this and additional works at: https://ir.library.louisville.edu/etd

Part of the Curriculum and Instruction Commons

\section{Recommended Citation}

Stone, Shannon M., "The impact of an interactive technology application on elementary student achievement in fractions." (2015). Electronic Theses and Dissertations. Paper 2298.

https://doi.org/10.18297/etd/2298

This Doctoral Dissertation is brought to you for free and open access by ThinkIR: The University of Louisville's Institutional Repository. It has been accepted for inclusion in Electronic Theses and Dissertations by an authorized administrator of ThinkIR: The University of Louisville's Institutional Repository. This title appears here courtesy of the author, who has retained all other copyrights. For more information, please contact thinkir@louisville.edu. 
THE IMPACT OF AN INTERACTIVE TECHNOLOGY APPLICATION ON ELEMENTARY STUDENT ACHIEVEMENT IN FRACTIONS

By

\author{
Shannon M. Stone \\ B.A., University of Kentucky, 2003 \\ M.A.T., Spalding University, 2005
}

\begin{abstract}
A Dissertation Submitted to the Faculty of the
College of Education and Human Development of the University of Louisville in Partial Fulfillment of the Requirements

for the Degree of
\end{abstract}

Doctor of Philosophy in Curriculum and Instruction

\author{
Department of Teaching and Learning \\ University of Louisville \\ Louisville, KY
}

December 2015 
Copyright 2015 by Shannon M. Stone

All rights reserved 



\title{
THE IMPACT OF AN INTERACTIVE TECHNOLOGY APPLICATION ON ELEMENTARY STUDENT ACHIEVEMENT IN FRACTIONS
}

\author{
By \\ Shannon M. Stone \\ B.A., University of Kentucky, 2003 \\ M.A.T., Spalding University, 2005
}

A dissertation approved on

November 30, 2015

by the following Dissertation Committee:

Karen S. Karp, Dissertation Director

\begin{tabular}{l}
\hline Elizabeth Todd Brown \\
\hline
\end{tabular}

Robert Ronau

Maggie McGatha

Timothy Landrum 


\section{DEDICATION}

This dissertation is dedicated to my husband,

\section{Jason Stone}

who has only known me as a doctoral student

and married me anyway

and

to my parents,

who each in their own way

have propelled me along in this journey. 


\section{ACKNOWLEDGEMENTS}

This journey started many years ago with Dr. Brenda Overturf. She asked me if I had ever given thought to pursuing my doctorate degree. Right then a seed was planted. Later, but many years before I started the program, I had met Dr. Karen Karp and she watered that little seed. While I had so many things to get right in my life, I knew where I was headed. This was a journey that I wanted to complete with all of my heart. I wouldn't have arrived at this destination without the help of many.

First, I have to give my deepest gratitude to my chair, Dr. Karen Karp. Not only did she water that seed initially, she has stuck out the journey until the end. She has provided so many wonderful opportunities through this program, that I truly feel that I have had unmatched experiences in comparison with others from different programs. Dr. Karp has a knack for weaving support circles that have not only led to magnificent professional experiences, but also deep personal relationships with others that have been on the same journey. Dr. Karp is an amazing example of a true leader.

Part of Dr. Karp's circle weaving brought me to Dr. Todd Brown. Dr. Brown not only serves on my committee, but she has also helped to shape the doctoral student that I was to become. Working with Dr. Brown several summers in the Summer Portfolio Institute offered me my first experiences leading graduate students. The time spent in Summer Portfolio Institute laid the groundwork for state and regional presentations that at one time I didn't have the guts to give. I also owe Dr. 
Brown the greatest thanks in offering her time and expertise in assisting getting my scatter brain together on this document. She helped me to reorganize and rethink what I was writing and gave critical thought to my work. Dr. Brown is truly a teacher, she knows exactly how to offer valuable feedback, but cushion her words so that you feel like you can take on the world!

I also owe great thanks to my committee members, Dr. Robert Ronau, Dr. Tim Landrum, and Dr. Maggie McGatha. Dr. Ronau flipped my world upside down many summers ago. From his Advanced Research Design class, I knew my academic world would never be the same. Dr. Landrum helped to initially get this project off the ground, many Monday nights around the whiteboard. Dr. McGatha has made me think critically about qualitative methods and mixed-methods research. Without each of them and their thoughtfulness this milestone would not occur.

In addition to my academic circle I wouldn't have survived this adventure without those peer supports that Dr. Karp put in place years ago when we all first started. Leah Dix-White, Liz Popelka-Brown, and Victoria Miller Bennett, have literally been my sanity savers over the years. They are the people who "get it," when everybody else thought I was an insane person. I wouldn't have done this journey without them and all those Saturday's locked up at dissertation camp. We made it girls! For the finisher before us, Sarah Bush, you paved the way and have continued to share your wisdom so that we can finish.

I owe gratitude to Diane Drescher and Bonnie Koontz for not only being my biggest cheerleaders but also for all the hours we put into grading all those assessments. I owe gratitude to Caitlyn Moser for taking "all those assessments" and 
coding and entering them into a workable data file. I owe thanks to Maria Clemons, Kevin Garner, and Tara Gagel for being willing participants in this adventure and making it happen at your schools. Professionally, I also owe gratitude to Danielle Holmes, Mohit Bhargava, and Ganesh Kumar for fielding my technology freak-outs and making things happen with the application. You all were amazing!

Lastly, and probably the greatest thanks goes to my family and friends outside of this. My husband has had to live with the insane person all these years. My kids Connor, Cooper, and Cheyenne for understanding that "I have to write." To my mom, who listened to me have a meltdown - countless times. To my brother Randy who understood that this was only short-term. For Lindsay, who also has understood this was temporary. To Denise, who has kept me in the rocker many times. And lastly to Lawanda, for not only rallying and praying, but who has also been forgiving of the sacrifice I have had to make to cross the finish line. I am forever indebted to everyone's patience, compassion, and understanding the last years. Thanks, be to God! 


\author{
ABSTRACT \\ T+( I0 3\$ \&7 2) \$1 I1 7( 5\$\&7,9( T\$\% ( 7 A33/,\&\$ 7,2 121 \\ S78 ' ( 17 A\&+, ( 9 ( 0 ( 1 7ロ, 1 F5 \$ \&7,2 16 \\ Shannon M. Stone
}

November $30 \square 2015$

Data reported locally and nationally show that students struggle to understand fractional concepts. The purpose of this study was to examine the effects of an interactive tablet application on student achievement in fractions. Students in grades three through five at the treatment school worked with an interactive tablet application designed to facilitate student construction of fractions knowledge. The interactive tablet application is an open-platform that allowed teachers the autonomy to work with the researcher to load their desired content activities on the topic of fractions to develop instructional sessions for students. Students worked independently during the math workshop to complete ten instructional sessions on fractions using the tablet application. These instructional sessions allowed students to construct responses to help develop better conceptual understanding. Students in the control group completed instructional activities without the use of the technology application.

This study used a quasi-experimental design with an untreated control group with dependent pretest and posttest samples. The study used the Reformed Teaching Observation Protocol (RTOP) to examine differences in observed teaching and 
learning environments between the treatment and control groups using a repeated measures one-way analysis of variance. The results of these observations indicated that the learning environments of the treatment and control groups did not differ significantly $(p=.262>.05)$. Thus, narrowing the effects to the differences in treatment. Power was established with six groups (2 classrooms at each of grades three, four and five). A minimum sample size of 162 participants was required to have adequate power with an effect size of .4 , alpha level at .05 , power at .95 , and because this study had 258 participants it met that level. Student knowledge of fractions prior to the treatment was examined with pretests and analyzed using an analysis of covariance (ANCOVA). Posttest scores of students' fractional knowledge after the treatment (for both treatment and control groups) were also analyzed using an ANCOVA. The results of the pretest analysis established that students' prior knowledge was a good indicator of their performance on the posttest. Findings on posttest scores between treatment and control revealed that overall in grades three through five, no differences in treatment and control groups were significant $(p=.140$ $>.05)$. Therefore, the treatment, interactive tablet application, had no impact on student proficiency results in fractions concepts. However, when examining interactions in the groups an effect was found significant $(p=.03<.05)$ at grade level. A difference in treatment and control groups was observed at the fifth grade level. The treatment effect, interactive tablet technology, did have a statistically significant effect on students' posttest scores $(p=.02<.05)$.

The results of this study provided an answer to the impacts an interactive tablet application have on student achievement in fractions. The implications of this 
study may require additional research with more in-depth observations of teacher technological pedagogical content knowledge (TPACK), student engagement in technology, and the effects of teacher feedback to students. Technology is a rapidly changing field and efforts to determine effective strategies in instruction with technology are a growing research need. This study adds to the current field of knowledge by offering research on an open-platform tablet application and its effects on fraction achievement. 
Organization of the Dissertation....................................... 15

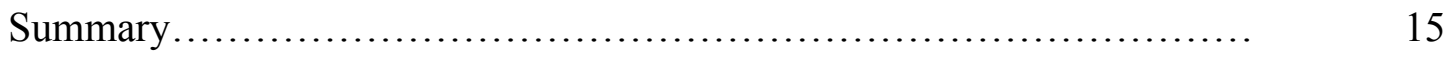

CHAPTER II: LITERATURE REVIEW ................................ 16

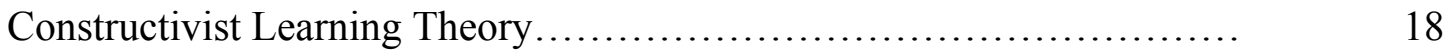

Constructivist Learning Theory and the Mathematics Classroom....... 20

Constructivist Learning Theory and the Integration of Technology...... 21

Pedagogical Content Knowledge......................................... 24

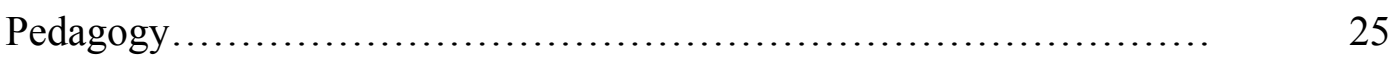

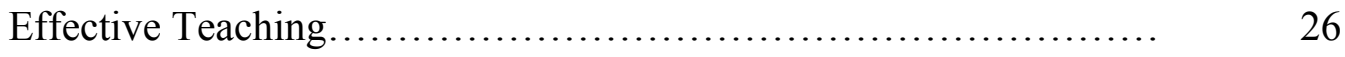

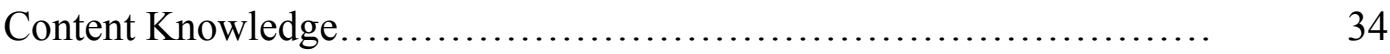

Curriculum......................................................

Fractions.......................................................... 36

Developing Understanding in Fractions........................ 41

Contexts to Support Fractions Learning.......................

Student Progress and Proficiency................................ 45

Technological Pedagogical Content Knowledge (TPACK)............... $\quad 50$

Importance of Technology Integration............................ $\quad 51$

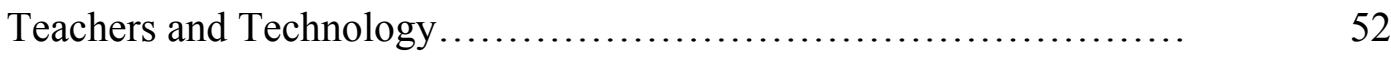

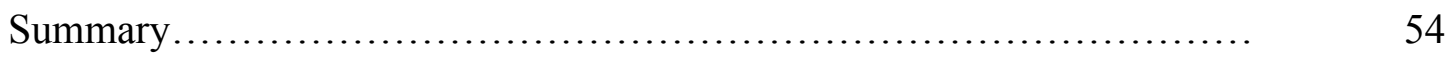

CHAPTER III: METHDOLOGY .................................... 55

Rationale ....................................................... 55

Research Question........................................... 55

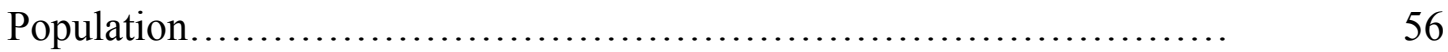


Treatment...................................................... $\quad 62$

Lesson Development........................................ 65

Lesson Overview........................................ 66

Student Preparation to use GoClass............................. 75

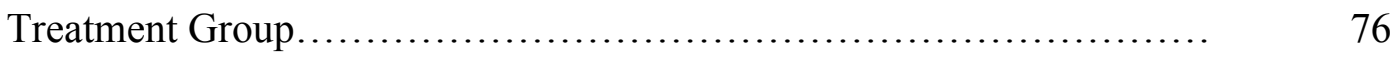

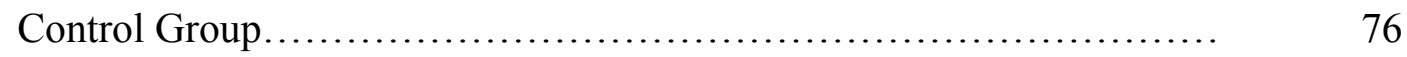

Research Design............................................. 78

Variables.................................................. 83

Independent Variable..................................... 83

Dependent Variable...................................... 83

Co-variate............................................... 83

Power Analysis............................................. 83

Measures...................................................... 85

Student Achievement.......................................... 85

GoMath Assessments for Student Achievement.................. 86

Rationale for Curriculum Based Assessment................... 89

Classroom Instruction and Mathematics Practice................. 90

Rationale for Using Classroom Observation.................... 93

Validity and Reliability.................................... 93

Statistical Analysis............................................. 94

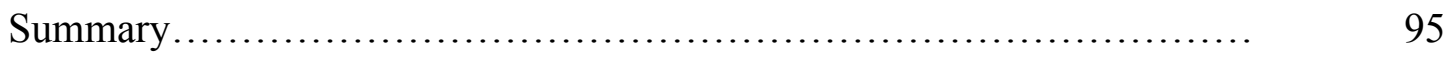


CHAPTER IV: RESULTS ....................................... 96

Purpose and Research Question...................................... $\quad 97$

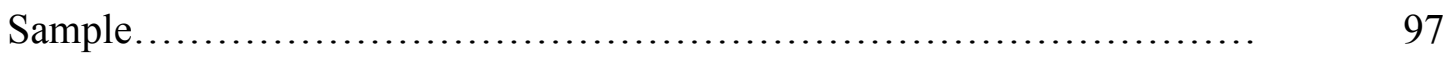

Teaching and Learning Environment................................. 98

RTOP Scores.................................................. 99

Overall Observation......................................... 99

Third Grade Observations........................................ 100

RTOP Raw Scores............................................ 101

Fourth and Fifth Grade Observations................................ 103

RTOP Raw Scores........................................... 104

Fourth Grade............................................ 104

Fifth Grade.....................................................

Summary of Teaching and Learning Environment...................... 105

Fidelity of Implementation........................................ 106

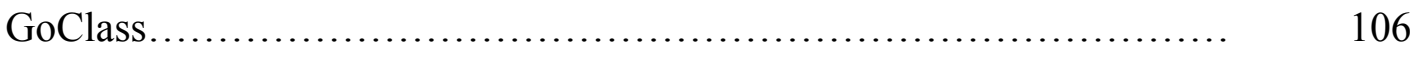

Reliability of GoMath: Common Core Edition......................... 108

Third Grade GoMath: Common Core Edition....................... 108

Fourth Grade GoMath: Common Core Edition........................ 108

Fifth Grade GoMath: Common Core Edition........................... 109

Summary of Reliability .......................................... 109

ANCOVA Analysis.................................................. 109

ANCOVA Assumptions.......................................... 109

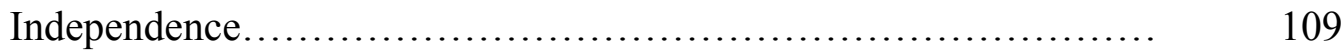


Normality ................................................... 110

Equal Variance............................................. 112

Independence of the Covariate................................. 112

Homogeneity of Regression of Slopes......................... 112

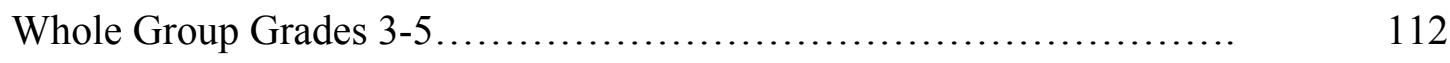

Descriptive Statistics......................................... $\quad 112$

ANCOVA.................................................. 113

Third Grade........................................................ 114

ANCOVA.................................................... 115

Fourth Grade ................................................... $\quad 115$

ANCOVA..................................................... 116

Fifth Grade............................................................. $\quad 119$

ANCOVA.................................................... 119

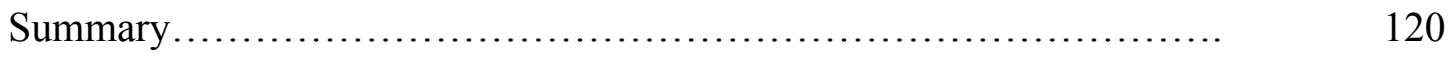

CHAPTER V: SUMMARY OF THE STUDY ......................... 122

Restatement of the Problem........................................ 122

Restatement of the Purpose and Research Question..................... 123

Review of Methodology and Approach to Analysis....................... 123

Summary of Findings............................................... 124

Discussion and Implications for Mathematics Education................. 125

Conceptual Framework........................................... 125

Constructivist Learning Theory and Interactive Technology............. 125

TPACK and Interactive Technology ............................. 126 


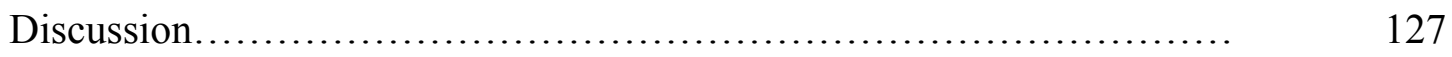

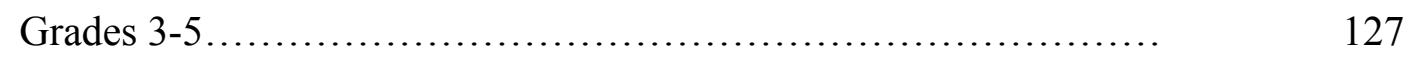

Third Grade........................................................... 128

Fourth Grade.................................................. 129

Fifth Grade....................................................... 130

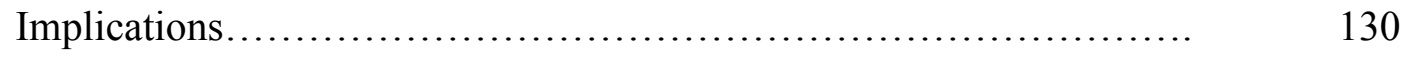

Students' Current Level of Understanding........................ 131

Teachers' Current Level of TPACK Understanding................. 131

Limitations and Recommendations.................................. 132

Technology Limitations............................................ 133

Teacher Limitations.................................................

Content Limitations............................................... 137

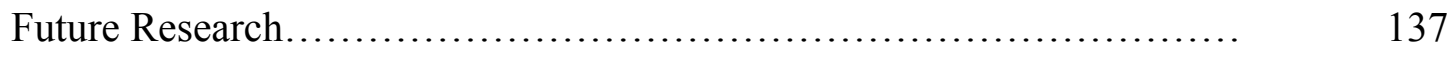

Conclusion.................................................... $\quad 140$

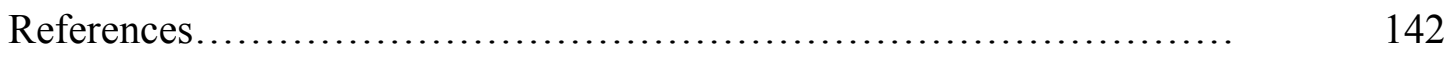

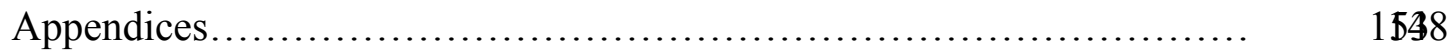

Curriculum Vita................................................. 157 


\section{LIST OF TABLES}

PAGE

2.1. Nine Effective Teaching Strategies......................... 28

2.2. High-Leverage Teaching Practices from the University of

Michigan................................................ 30

2.3. Common Core Standards Mathematical Practices................ 32

2.4. Common Core State Standards Mathematics Fractions and the

Trajectories........................................ $\quad 39-40$

2.5. Mathematical Proficiency................................. 47

2.6. Relationship Between Proficiency Strands and CCSS

Mathematical Practices.......................................

3.1. Enrollment of Population and Percentage of Free and Reduced

Lunch............................................... 56

3.2. Demographic Information of Sampled Schools.................. 57

3.3. Classes and Students in Each Group........................ 58

3.4. District Data on Assessed Fractions Skills...................... 60

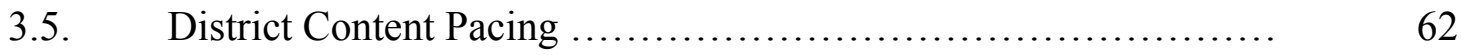

3.6. Outline of Treatment GoClass Lessons........................ 67-68

3.7. Study Schedule........................................ 82 
3.8. GoMath: Common Core Edition (2011) Assessment Guide

Chapter Tests............................................... 89

3.9. Reformed Teaching Observation Protocol (RTOP) Categories...... 91

4.1. The Number of Pre-Post-Test Scores for Treatment and Control.... 98

4.2. Repeated Measures for Differences Between Treatment and

Control................................................ 100

4.3 Repeated Measures for Differences Between Treatment and

Control................................................. 101

4.4. Third Grade RTOP Scores for Treatment and Control Groups...... 102

4.5. Repeated Measures for Differences Between Treatment and

Control..................................................... 103

4.6. Fourth Grade RTOP Raw Scores for Treatment and Control

Groups................................................. 104

4.7. $\quad$ Fifth Grade RTOP Raw Scores for Treatment and Control

Groups............................................. 105

4.8. Reformed Teaching Observation Protocol (RTOP) Categories...... 106

4.9. Summary of Cronbach Alpha Coefficients for Reliability.......... 108

4.10. Posttest Descriptive Statistics for Treatment and Control

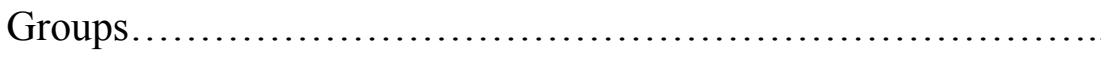

4.11. Analysis of Covariance Treatment and Control Groups........... 114

4.12. Third Grade Analysis of Covariance Posttest Scores.............. 115

4.13. Fourth Grade Analysis of Covariance Posttest Scores............ 117

4.14. Fifth Grade Analysis of Covariance Posttest Scores............... 119 
5.1. Treatment and Control Group Posttest Mean Comparison......... 


\section{LIST OF FIGURES}

PAGE

1.1. Conceptual Framework …............................... 6

1.2. TPACK Framework ….................................... 8

2.1. Four Factors in Promoting Proficiency in Number and Operations Fractions .................................................... 17

2.2. The Constructivist Learning Environment ..................... 23

2.3. Pedagogical Content Knowledge Diagram ...................... 25

2.4. Case Example of Fraction Rules ........................... 45

2.5. TPACK Framework …...................................... 51

3.1. Screen Shot GoClass Lesson Outline .......................... 68

3.2. Screen Shot GoClass "Show" Screen for Students ................ 69

3.3. Screen Shot Go Class "Explain" Screen for Students .............. 70

3.4. Screen Shot GoClass "Ask" Screen for Students ................. 71

3.5. Screen Shot Go Class Final Multiple Choice Questions ........... 72

3.6. Kidspiration Question Set-up Example ....................... $\quad 73$

3.7. Kidspiration Question Answer Example ....................... $\quad 74$

3.8. Untreated Control Group Design With Dependent Pretest, Posttest

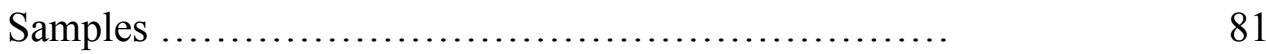

3.9. Power Analyses ........................................ 84 
3.10. GoMath: Common Core Edition (2011) Form A Question

Examples

$87-88$

3.11. Items on the Reformed Teaching Observation Protocol (RTOP) .. $\quad 92$

4.1. Histogram of Third Grade Posttest Scores..................... $\quad 110$

4.2. Histogram of Fourth Grade Posttest Scores.................... 111

4.3. Histogram of Fifth Grade Posttest Scores....................... 111

5.1. TPACK Knowledge Levels of Teachers........................ 136 


\section{$\&+\$ 37(5 \square$}

$$
29(59,(:
$$

This study examined student achievement on fractional concepts after the students engaged in interactive fractions lessons using an interactive application on a tablet device.

\section{Problem Statement}

Elementary school students struggle to understand fractional concepts (Battista, 2012; Brown \& Quinn, 2007; Chval, Lannin, Doughtery, \& Jones, 2013; Kilpatrick, Swafford, \& Findell, 2001; McNamara \& Shaughnessy, 2010; NAEP, 2000; Petit, Laird, Marsden, \& Ebby, 2015). The use of technology in mathematics has shown to positively increase student achievement (Herrera, Kanold, Koss, Ryan, Speer, 2007; Holmes, 2009; International Society of Technology Education (ISTE), 2007; Kent, 2006; Manzo, 2009; Marzano \& Haystead, 2009; Means, \& Olson, 1994 ; Morphew, 2012; National Council of Teachers of Mathematics (NCTM), 2000). Therefore, allowing students to explore fractional concepts using technology could increase student achievement in this area.

\section{Background}

Common Core Standards for Mathematics (National Governors Association Center for Best Practices [NGA Center], 2010) have identified five domains 
important in students' mathematical learning in grades three through five. These mathematical domains include:

- operations and algebraic thinking

- number and operations in base ten

- number and operations: fractions

- measurement and data and

- geometry.

Within these five domains of learning, students still struggle with fractional concepts (Brown \& Quinn, 2007; NAEP, 2000). Results from the National Assessment of Educational Progress: Seventh Mathematics Assessment (2000) showed that 54\% of fourth graders could not "identify a fraction $\frac{n}{d}$ representing an indicated part of a set..." and $16 \%$ of fourth graders could not "explain why one unit fraction is larger or smaller than another...". This struggle to understand fractional concepts has implications for learning future mathematical concepts (Brown \& Quinn, 1997).

This study will focus on fractions for several reasons. The most viable argument to focus on fractional content is the connection to algebraic concepts. Barnett-Clarke, Fisher, Marks, Ross \& Zbiek (2010) explain that, The study of algebra is impossible without an understanding of fractions. Besides important ideas involving proportional reasoning that come up in algebraic contexts (see Lobato and Ellis, 2010), almost all instances of division in algebra are represented as fractions, and to work well with any of these expressions, we must have knowledge of fractions (p. 10). 
Placing an importance on the teaching and learning of fractional concepts in the elementary grades is critical for student success in future grades, especially in algebraic concepts. Brown and Quinn's (2007) study of 191 students ranging from elementary to high school examined the effect of students' fractional understanding on their knowledge of algebra concepts. After testing concept knowledge and computational fluency, Brown and Quinn (2007) found that "the "average" elementary algebra student, of the sample population, as neither proficient nor familiar with operations and fraction concepts" (p. 11). The implications of Brown and Quinn 's (2007) study also suggests that students' understanding of fractions in fourth grade positively impact future understanding of algebraic concepts.

The focus on fractions for elementary students is important because concepts centered on rational numbers can be more challenging than other concepts (BarnettClarke, Fisher, Marks, Ross, \& Zbiek 2010; Kilpatrick et al., 2001). Students often have a difficult time transitioning between whole numbers and rational numbers and struggle to recognize fractional parts in relation to the whole or identify the unit they are describing (Barnett-Clark et al., 2010; Kilpatrick et al., 2001; Petit et al., 2010). Addressing these gaps requires teachers to look for meaningful ways to engage students in learning fractional concepts that will help them build stronger understanding.

Teachers may choose technology as a way to engage students in rational numbers concepts. Technology invites exploration in problem solving and can help students develop thinking and create innovative products to demonstrate their knowledge of content (ISTE, 2007; Morphew, 2012). Students who interact with 
personal digital devices, interactive whiteboards, and other emerging technologies have been found to demonstrate an increase in engagement, as well as an increase in achievement (Holmes, 2009; Kent, 2006; Marzano \& Haystead, 2009; Means, \& Olson, 1994). Marzano and Haystead (2009) found in a study of 85 teachers and 170 classrooms in grades K-12 that "using interactive whiteboards was associated with a 16 percentile point gain in student achievement" (p. 65). This magnitude of gain in student achievement reinforces that students should use technology in ways that extend learning experiences with engaging and meaningful interactions during mathematics instruction.

Mathematics instruction utilizing technology should cause students to reason and expand their thinking regarding mathematical concepts and support and extend mathematical learning (Jonassen, 2000; Morphew, 2012; NCTM, 2000; NCTM, 2008; Niess et al., 2009). This study attempts to demonstrate how technology can be effectively integrated into mathematical instruction of fractional concepts in elementary classrooms. Using an interactive application integrated with fraction lessons will support students' construction of fractional knowledge by giving them opportunities to reason and problem solve about fractional concepts.

\section{Conceptual Framework}

The framework for this study is organized into five areas:

- constructivist learning theory

- technology pedagogy content knowledge (TPACK)

- interactive technology

- instructional strategies with a tablet device 
- student proficiency in the domain of Numbers and Operations: Fractions (NGA Center, 2010).

Figure 1.1 demonstrates the relationship between each of the five areas of the framework. The first three areas, constructivist learning theory, TPACK, and interactive technology describe the development of learning activities and teacher roles in student learning with technology. These three areas define the strategies used and the level of integration of tablet devices in instruction. Figure 1.1. also demonstrates that all four of these areas may impact student proficiency in Numbers and Operations: Fractions (Marzano \& Haystead, 2009; Mishra \& Koehler, 2006; NGA Center, 2010; Shulman, 1986). 
Figure 1.1. Conceptual Framework

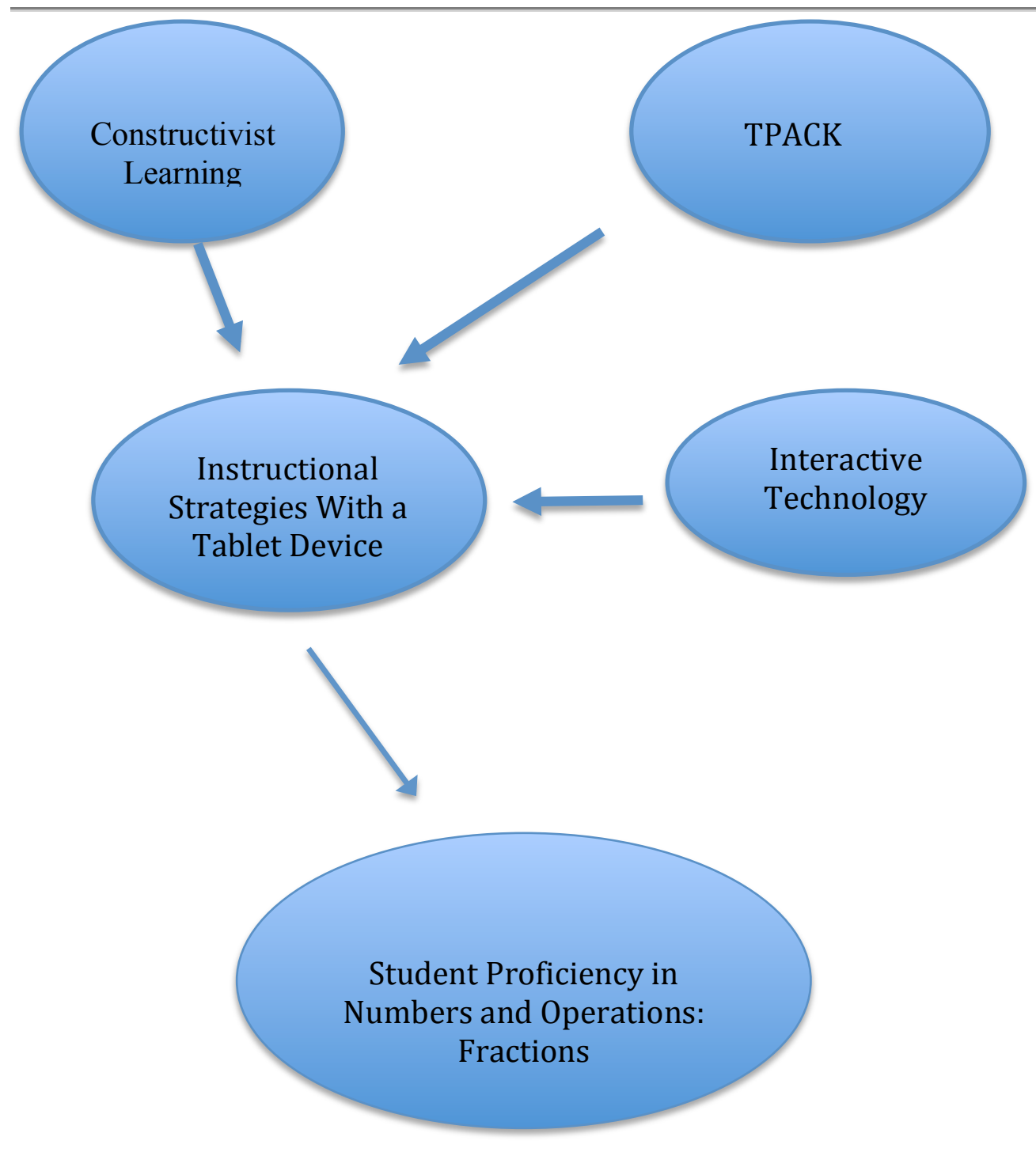

Figure 1.1. The conceptual framework diagram illustrates the areas that impact teacher choices when developing activities for students to complete on a tablet and their impacts on student proficiency in Numbers and Operations: Fractions.

Constructivist Learning Theory. Piaget's (1954) theory of constructivism suggests that students construct knowledge through cognitive organization. All learners enter into any situation with a defined level of knowledge about the situation and each new idea is understood through this previous learning or schema (Piaget, 
1954). Using that knowledge set, students have opportunities to construct their own knowledge about a particular topic. Knowledge acquired through new situations either extends the previously defined knowledge or helps to reformulate that knowledge set (Piaget, 1954; Vygotsky \& Cole, 1978). Students construct knowledge from these schemas and the integration of new experiences.

In this study constructivism is the foundation of learning activities completed on a tablet device. In Figure 1.1., an arrow between constructivism and a tablet device represent the strategies and the possible relationship between the two. The theory of constructivism informs lesson development for the interactive tablet application to help assist students in learning fractional concepts. In this study, students construct answers to real-world problems using their previous understanding of equipartitioning (Chval et al., 2015; Fosnot \& Dolk, 2002; Hennessey et al., 2012; Morphew, 2012). Students will acquire new knowledge about fractions using the interactive application on a tablet device.

TPACK. The TPACK framework demonstrates the fluidity of pedagogy, content knowledge, and technology (Mishra \& Koehler, 2006). Figure 1.2. illustrates the TPACK framework and the overlapping components of pedagogy, content knowledge, and technology. Notice that technology is not a separate entity used outside of content or pedagogical practice but is an integral component. Technology is blended within these areas to develop learning activities where students are actively engaged in learning using digital tools (Mishra \& Koehler, 2006). 
Figure 1.2. TPACK Framework

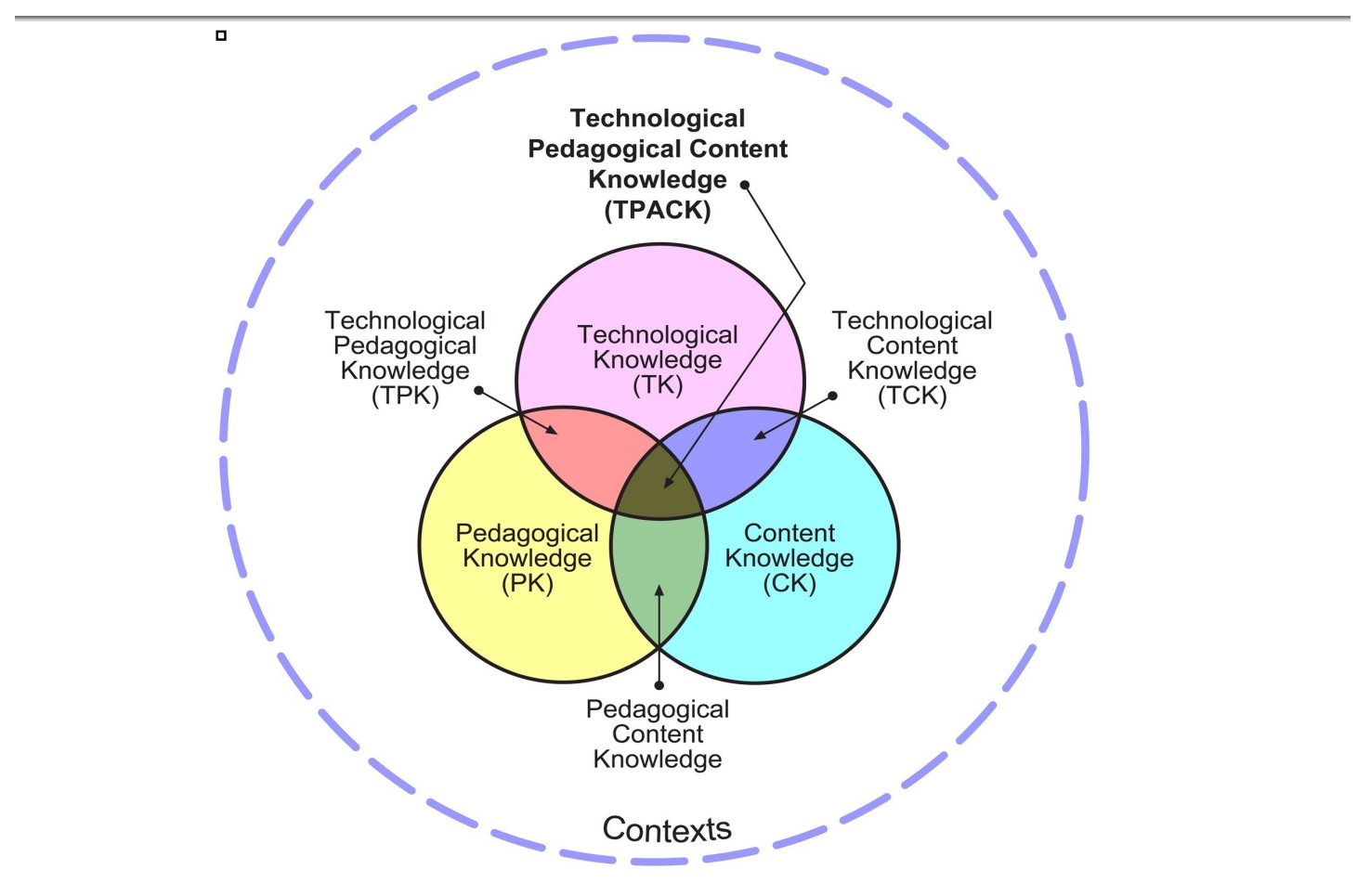

Figure 1.2. Mishra and Koehler's graphic representation of the TPACK framework (http://tpack.org, 2006).

One goal of this study is to explore and apply the TPACK framework when designing mathematics lessons that will increase student proficiency in learning fractional concepts. In Figure 1.1 an arrow between TPACK and tablet strategies represents the relationship between the two. TPACK makes evident the knowledge required by teachers to effectively integrate technology in their classroom (Mishra \& 
Koehler, 2006). This knowledge level may determine how well a teacher will use the tablet application in his or her instruction of fractional concepts. In this study the researcher acted as a technology facilitator to help expedite the use of the tablet application in the mathematics classroom (Reinhart, Thomas, \& Toriskie, 2011). The use of a technology facilitator will support teachers' knowledge in the integration of technology in both the content and pedagogical process.

Interactive Technology. Twenty-first century teaching with digital tools entails teachers integrating technologies into their instruction with the idea that this will support engagement and interest by students. This approach to infusing technology in teaching embraces all aspects of TPACK (Koehler \& Mishra, 2008). These technologies might include tablets, gaming, social networking, and digitalmedia creation. Students who interact with personal digital devices, interactive whiteboards, and other technologies have increased levels of engagement, as well as increased levels of achievement (Holmes, 2009; Kent, 2006; Marzano \& Haystead, 2009; Manzo, 2009; Means, \& Olson, 1994).

Technology is consistently changing and new technologies are introduced and researched in the classroom. This research base of interactive technology helps to inform how tablets may be positively integrated into fractional concept instruction to maximize student engagement. In Figure 1.1., this relationship is represented with an arrow between interactive technology and instructional strategies with tablet devices.

Instructional Strategies with Tablet Devices. An EbscoHost search on "iPad strategies for mathematics" revealed that there is limited empirical research or information about strategies that can be used to effectively integrate iPad's into 
mathematics instruction. "Mathematics and tablet technology" return similar results. The search for other research on technologies indicates that tools should be used in interactive ways that allow students to construct their understanding about mathematics concepts (Jonassen, 2000; Morphew, 2012; NCTM, 2000; NCTM, 2008; Niess et al., 2009). Therefore, in this study a tablet device will be used as a tool to extend elementary students' learning by allowing them to work with interactive fractional content through the manipulation of shapes and characters.

The instructional strategies with tablet technology used in this study are informed through three areas, constructivist learning, TPACK, and interactive technology. In Figure 1.1. the relationships of these areas are indicated with an arrow directed at instructional strategies with tablet devices. Constructivist learning helps to guide the types of activities that students will engage with on the tablet device. Constructivism goes beyond a didactic approach and promotes student opportunities to work with concepts (Piaget, 1954). In this framework instructional strategies using a tablet device are centered on allowing students to develop their previous fractions schema by giving them opportunities to problem solve (Chval et al., 2013; Empson, 2001; McNamara, \& Shaughnessy, 2010; Morphew, 2012). TPACK guides how well teachers will apply their technological knowledge inside the classroom with the use of the tablet devices (Mishra \& Koehler, 2006). The researcher will guide teachers on the frequency of use of tablet technology and specifically how to use the tablets inside their instruction (Reinhart et al., 2011). Research done previously in these three areas may possibly aid in the effective development of fractional concepts strategies through the use of tablet devices. 
Student Proficiency. In Figure 1.1., three constructs are used to inform tablet strategies. These strategies developed on a tablet are used to assist students in learning fractional concepts. Student proficiency will be measured by the impact these instructional strategies with tablet devices may have on their fractional learning. This relationship is indicated in Figure 1.1. with an arrow between tablet strategies and Student Proficiency: Number and Operations: Fractions. This approach to integrating tablet technology into instruction with fractions is expected to impact student proficiency in fractions for several reasons. First, allowing students to construct their knowledge about mathematics increases conceptual development (Chval et al., 2015; McNamara \& Shaughnessy, 2010; NCTM, 2000). Second, technology can increase student engagement and as a result increase student achievement (Holmes, 2009; Kent, 2006; Marzano \& Haystead, 2010; Manzo, 2009; Means \& Olson, 1994). This increase in conceptual development and engagement directly impacts proficiency (Kilpatrick, Swafford, \& Findell, 2001; Marzano \& Haystead, 2009; Means \& Olson, 1994).

Student understanding can be more closely defined using proficiency measures. Kilpatrick et al. (2001) measure mathematical proficiency in five strands:

- conceptual understanding

- procedural fluency

- strategic competence

- adaptive reasoning

- productive disposition (p. 5). 
These strands overlap and intertwine to offer a well-rounded approach to mathematical understanding. These proficiencies are effectively accomplished with high levels of student engagement in rigorous instruction and learning activities (Kastberg \& Frye, 2013).

\section{This Specific Study}

\section{Purpose}

The purpose of this study is to examine the impact of an interactive tablet application on student achievement in fractions. Students were provided the opportunity to construct their own knowledge using the interactive tablet application, to support their achievement in fractional concepts (Means \& Olson, 2004; Manzo, 2010; \& Marzano \& Haystead, 2009).

\section{Research Question}

How will elementary student achievement be impacted by the use of an interactive tablet application designed to facilitate student construction of responses to fraction problems?

\section{Overview of Methodology}

This study is a quasi-experimental design with an untreated control group with dependent pretest and posttest samples. Shadish, Cook, and Campbell (2002) describe quasi-experiments as "experiments' that lack random assignment of units to conditions but that otherwise have similar purposes and structural attributes to randomized experiments" (p. 104). Academic achievement will serve as the 
dependent variable and instructional methods will serve as the independent variable. This study occurred in two elementary schools in grades three through five. The treatment, interactive tablet application, was administered at one school in grades three through five. The second school was the control group and had no interactive tablet application administered in grades three through five. A pretest was given to account for the current level of knowledge for each student and as such served as the covariate. A one-way analysis of covariance was used to determine the effects of an interactive tablet application that allows students to construct their answers on student achievement in fractions. To control for instructional differences across treatment and control groups, classroom observations were completed using the Reformed Teaching Observation Protocol (RTOP). The RTOP provided evidence that pedagogical practices and learning environments (constructivist practices) were comparable across groups.

\section{Significance of This Study}

At the start of this study research on tablet devices and their impact on mathematics achievement was limited. When searching EbscoHost database under the terms "tablets and mathematics and elementary" only four articles are returned; and of the four returned only three are relevant to this study. Searches completed on EbscoHost using the terms "tablets and mathematics" return minimal relevant results with most written in relation to the medical field. When searching EbscoHost using the terms "iPads and mathematics" twenty-one results are yielded; of the yielded results only one article demonstrates the results of a statistical study. The remaining articles were practitioner oriented descriptions of applications suggested for 
classroom use, narratives on the possible effects this technology may have in the classroom, and dialogs considering the uses for iPad's in mathematics classrooms.

The results of this study provide important empirical insights to the way tablet technology could effectively be used inside an elementary mathematics classroom. This study also examines important ways that teachers can include tablet technology to aid in students' understanding of fractions.

\section{Delimitations}

This study was conducted in the spring of 2015 in a large urban mid-western school district. This study has several delimitations. First, this study was only conducted in grades three through five. This study does not examine the effects of interactive technology in mathematics for any other grade level. Second, this study only focused on students' learning of fractional concepts. Similar results may or may not be reproducible when looking at other areas of mathematics such as algebra or geometry. Third, this study only examined one interactive application on tablet devices inside the classroom. This application has several functions that include the ability to have students complete assignments on the application outside of school. This aspect of the application was not considered as part of the study. In addition, this application is not device dependent; students could interact with the application across devices including laptops or computers. This study did not examine the application on any other device. Lastly, this study was conducted inside a large urban mid-western school district and only compared the difference between two groups within these schools. This study may have different results if reproduced in a rural setting or in a larger sample. 


\section{Organization of the Dissertation}

This dissertation is organized into five chapters: introduction to the importance and relevance of the study, literature review of the study topic, methodology of the study, report of the results, and discussion and recommendation of the findings of the study. Chapter two includes a detailed literature review on the topic including fractional concepts, effective teaching, student proficiency, and interactive technology. Chapter three is organized to discuss the methodological design of the study. That chapter includes a description of the population, sample, variables, statistical methodology, and threats to the validity and reliability of the study. Chapter four presents the results of data collection. Chapter five discusses the findings of the study and will offer suggestions and implications of the study for future research and mathematics instruction in the elementary classroom.

\section{Summary}

Elementary school students struggle to understand fractional concepts (Battista, 2012; Brown \& Quinn, 2007; Chval et al., 2013; Kilpatrick et al., 2001; McNamara \& Shaughnessy, 2010; NAEP, 2000; Petit et al., 2015). Teachers can support student understanding of fractions through effective use of technology in instruction.

When looking at Figure 1.1, constructivist learning, interactive technology, and TPACK are areas of the instructional process that inform how to strategically use tablet technology to make an impact on student achievement in Numbers and Operations: Fractions. For this study, a tablet application is a tool used to support student understanding of fractions in grades three through five. 


\section{CHAPTER II}

\section{LITERATURE REVIEW}

This literature review is organized around the conceptual framework for the study. Beginning with TPACK, this literature review addresses the three main areas of the framework including technology, pedagogy, and content knowledge. In addition to addressing the TPACK framework this literature review discusses the important aspects of constructivist learning theory. Figure 2.1 shows the four areas of discussion in the literature review. TPACK represented by: T: Technology, PK: Pedagogical Knowledge, and CK: Content Knowledge. The order of discussion is outlined below. 
Figure 2.1 Four Factors in Promoting Proficiency in Number and Operations: Fractions

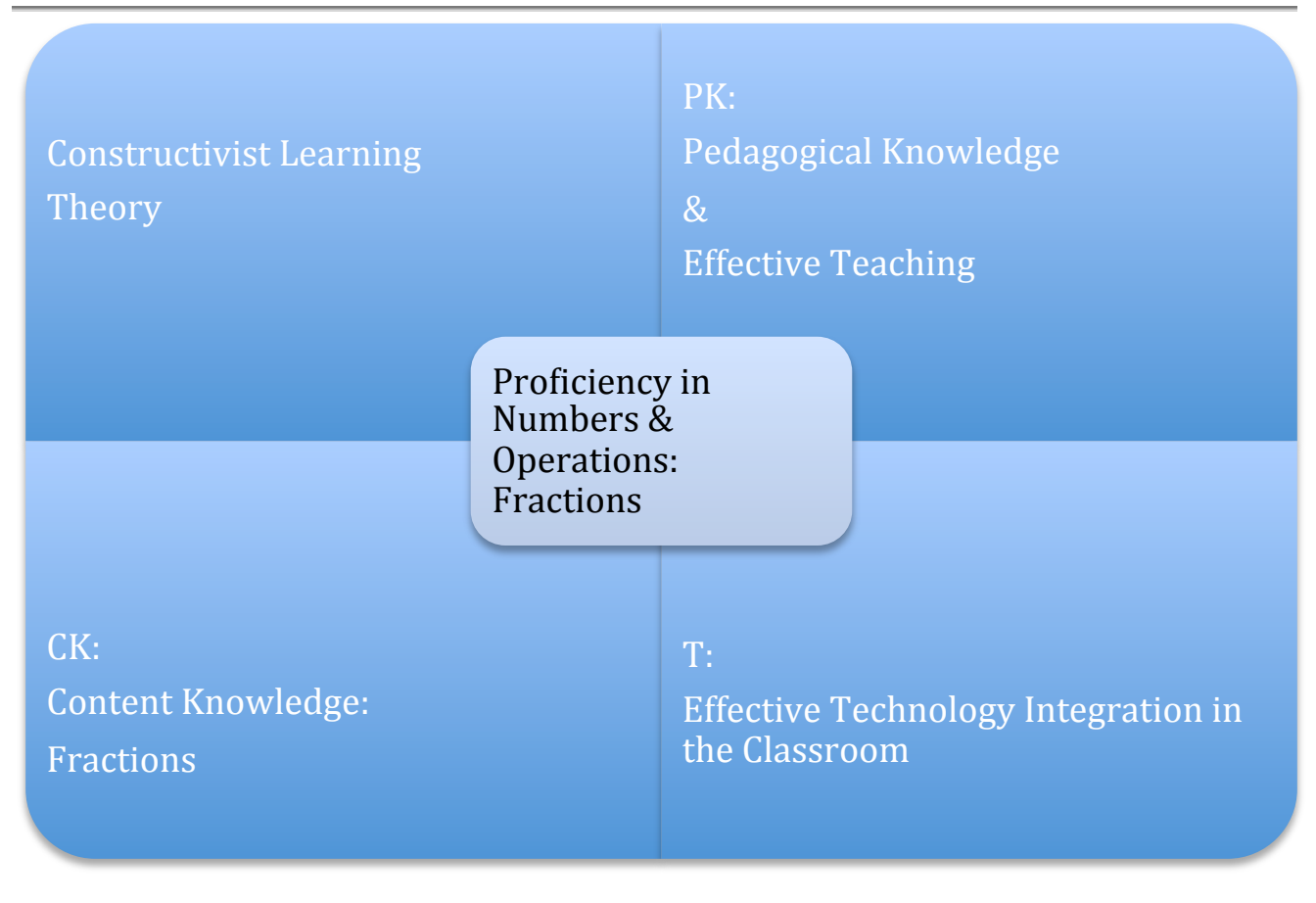

Figure 2.1 Four factors in promoting proficiency in Number and Operations with Fractions include the TPACK framework and constructivist learning theory.

The first area in the framework that this literature review addresses is constructivist learning theory. Teachers must understand how students learn before they can plan to teach. Constructivist learning theory suggests that students are active participants in their learning.

The second part of the framework this literature review is about pedagogy and the components of effective teaching. Accountability measures show that effective classroom instruction positively impacts student achievement (Marzano, Pickering, \& Pollock, 2001; Strong, Silver, \& Perini, 2001; University of Michigan, 2015). This review highlights how effective teaching is defined and how effective researchinformed teaching strategies appear in current literature. 
The third area this literature review discusses is the content knowledge needed for understanding fractional concepts and the requirements for student proficiency in fractions. Fractional concepts can be difficult for students to grasp (National Mathematics Panel Report, 2008). In this literature review fractions are defined as part of the rational number system, descriptions of the level of fractional understanding, and the experiences and practices that help students reach the desired level of proficiency. In addition, the levels of understanding in relation to Kilpatrick and colleagues' (2001) strands of mathematical proficiency will be discussed. As instruction occurs in the classroom students should reach a level of proficiency in which they can adequately demonstrate knowledge of fractional concepts and skills.

Lastly, the review presents effective methods to integrate technology in the classroom to enhance mathematics learning. Effective instruction examines many different strategies and approaches to learning. Teachers can expand effective instruction through the use of various tools including technology (Jonassen, 2000; Koehler \& Mishra, 2008; Morphew, 2012). The TPACK framework supports a constructivist approach to learning by highlighting areas in which teachers can effectively integrate technology in their instruction that allows students to be constructers of knowledge.

\section{Constructivist Learning Theory}

Constructivist theory suggests that learners build knowledge through experiences and cognitive organization (Piaget, 1954). Each new idea is understood through previous learning or schema (Piaget, 1954). Using that knowledge students have opportunities to construct their own learning about a particular topic. 
Knowledge acquired through new situations either extends the previously defined knowledge or helps to reformulate the previous knowledge (Piaget, 1954; Vygotsky \& Cole, 1978). Students construct knowledge from these schemas and the integration of new experiences.

Students in a constructivist classroom versus a didactic model interact with content through various experiences rather than receiving information directly from the teacher. These experiences may include hands-on manipulatives, or real-world experiences (e.g. ordering food from a restaurant, planning a trip, or working with live animals). In a constructivist classroom the focus shifts away from the teacher and onto the students' ability to develop and construct their ideas and learning. Creating a classroom environment in which students are offered the opportunity to problem solve, collaborate, and bring creativity to the classroom allows them to develop mathematical ideas in authentic ways (Dunham \& Hennessy, 2008; Means \& Olson, 1994; Morphew, 2012). Constructivist theory suggests that students have ownership of their learning because knowledge is created rather than given.

Vygotsky and Cole's (1978) learning theory is an extension of Piaget's ideas on building knowledge from previous experiences. Vygotsky and Cole (1978) recognized the importance of social interaction with other people in the learning process. They believed that people build knowledge through discussion of meaningful concepts with others, thus extending Piaget's ideas by including social interaction into the knowledge construction process. In the classroom, Vygotsky and Cole's (1978) social experiences would include those with other students as well as other adults, such as teachers, teaching assistants, and visiting classroom guests. 
Students actively participate in the planning and construction of learning through discourse and interactions with peers and teachers; through the discourse and interactions students set and meet specific learning goals (Toumasis, 2003). Teachers who create experiences that provide opportunities for student discourse and encourage them to interact with content in meaningful ways, helps students socially construct knowledge around targeted concepts.

Constructivist learning theory and the mathematics classroom. In a constructivist learning environment students develop a better conceptual understanding of mathematics (Chval et al., 2013; McNamara \& Shaughnessy, 2010; NCTM, 2000). Mathematical skill sets include ideas about numbers and operations such as counting and computation and continue through more sophisticated and complex concepts in algebraic thinking. As students work on their developed knowledge they are able to build their concrete understanding of mathematical skills. When students develop a concrete understanding of a concept they are able to move to a more semi-concrete, and finally an abstract understanding (Heddens, 1964; Witzel, 2005). The process of constructing knowledge from the concrete to the abstract promotes mathematical concept retention (Bandura, 1977).

When creating student-learning goals, several factors can help teachers facilitate students' construction of knowledge within the three levels (concrete, semiconcrete, and abstract) of mathematical understanding. These factors include developing experiences that allow students to generate their own powerful ideas, accommodate different learning styles, and provide access to a variety of media (Hennessey et al, 2012). Additional experiences include posing real-life mathematics 
problems for students to inquire about and solve. Real-life problems encourage students to take part in the process of constructing new learning and pushes them to move beyond the concrete foundation to a more abstract understanding.

\section{Constructivist learning theory and the integration of technology. As} students construct new knowledge they should also have the opportunity to learn in ways that prepare them as $21^{\text {st }}$ century digital citizens. A constructivist framework supports digital learning experiences. In The Constructivist Learning Environment Theory (CLE) (Jonassen, 1999) teachers should allow time for modeling and student discourse around standards-based problem solving that can occur in a digital environment. Jonassen (1999) believes that tools in the CLE theory should include technologies that will help scaffold learning in ways that promote problem context, collaboration, discourse, information, and authentic experiences.

In Figure 2.2 Jonassen's (1999) visual representation of CLE provides a framework for developing effective activity tasks in a constructivist classroom. Activity tasks may include digital components, which engage students in developing concept understanding. These tasks may be conducted in collaborative group or pair work, and consist of projects, activity sheets, or writing assignments. In the case of digital activity tasks, students' activity sheets would be replaced with interactive applications and writing assignments would be created using digital tools such as blogs, wikis, and digital organizing software or applications. A digital activity task is one done using a digital device; which may include the development of a graphic presentation, graphic representation using art software or digital sketchpads, the use of virtual manipulatives, or the use of software or applications that help develop a 
targeted skill. A digital environment involves any work done on technology devices such as computers and tablets Digital tasks should begin with the center circle of the CLE framework: problem/project context, problem/project representation, problem/project manipulation space.

When using technology effectively in the classroom, student activities should have many of the components outlined in Figure 2.2 (Jonassen, 1999). For example, technology experiences should include modeling and scaffolding support, as well as social interaction (Jonassen, 1999). Digital technology integration should push the learner to gather information from various sources, and sort that information in order to form their own perspectives and ideas on how to define those skills and concepts. Students' learning opportunities should include access to resources, tools, and contextual support within the digital environment. These opportunities promote effective technology integration inside a constructive classroom. 
Figure 2.2. The Constructivist Learning Environment

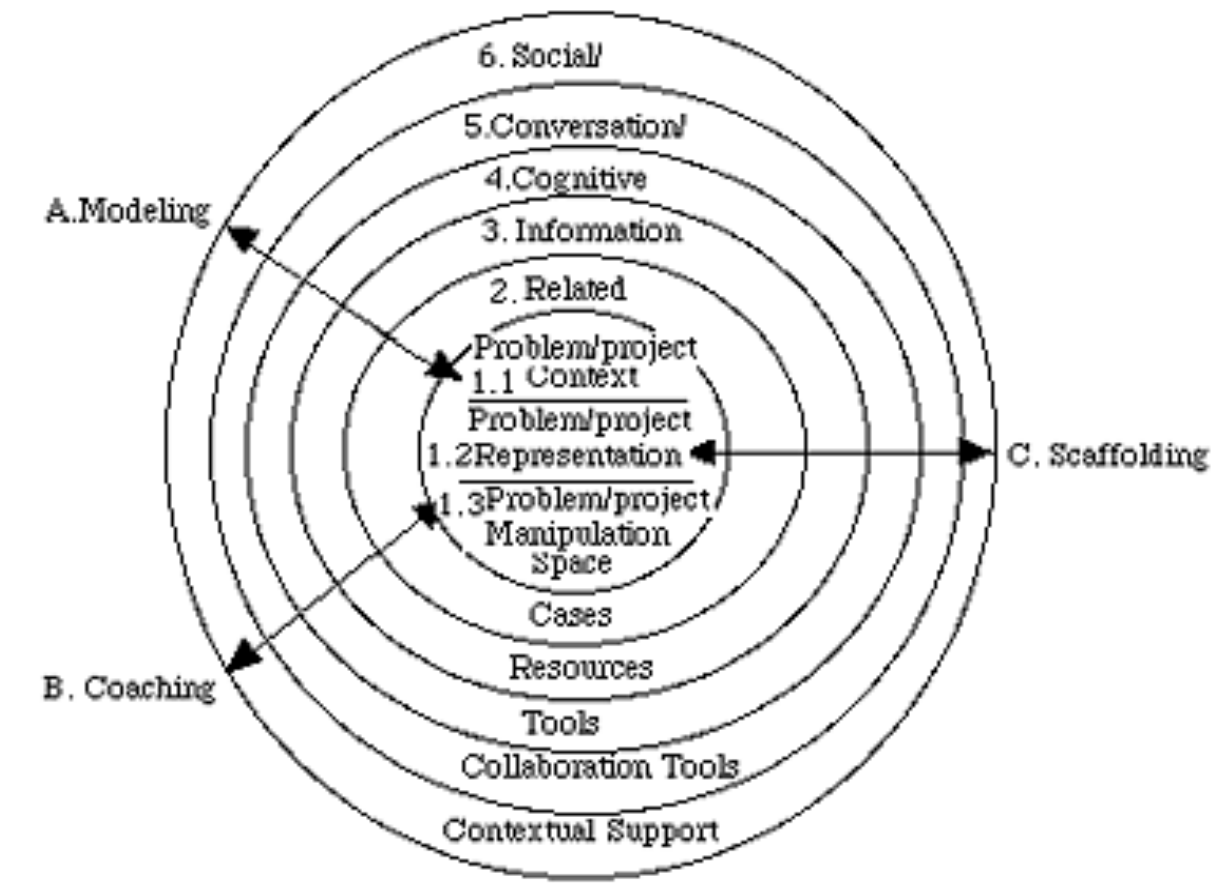

Figure 2.2 The Constructivist Learning Environment. (CLE) Theory (Jonassen, 1999).

The framework shows as students move outward from the center of the circle they are constructing their ideas and knowledge through the development of solutions to a particular problem. By using digital tools students develop their understanding and perform meaningful tasks collaboratively. In this environment constructivist teachers encourage reflection and promote interactive mathematical communication (Hennessey et al., 2012). In an effective constructivist approach to learning everyone shares common goals, equal partnerships, and collaborative relationships that lead to further success in learning. 


\section{Pedagogical Content Knowledge}

Teachers need knowledge on how students learn, methods for teaching, and up to date content about the subject they will teach. Shulman's (1986) ideas about teacher knowledge help to demonstrate the complexity of teaching. Figure 2.3 shows Shulman's (1986) Pedagogical Content Knowledge for teaching. Teaching encompasses the content knowledge required to teach as well as the pedagogy, or method to teach. Shulman $(1986 ; 1987)$ suggests that these areas of content knowledge and pedagogical knowledge cannot be treated as separate entities, but rather the blending of knowledge that will lead students to the appropriate conclusions about the subject matter. Teachers must have effective skills to determine the appropriate method for delivering content, thus pedagogical content knowledge is important in effective instruction (Shulman, 1987). Shulman's $(1986 ; 1987)$ work demonstrates the fluidity and importance of both pedagogy and content. 
Figure 2.3. Pedagogical Content Knowledge Diagram

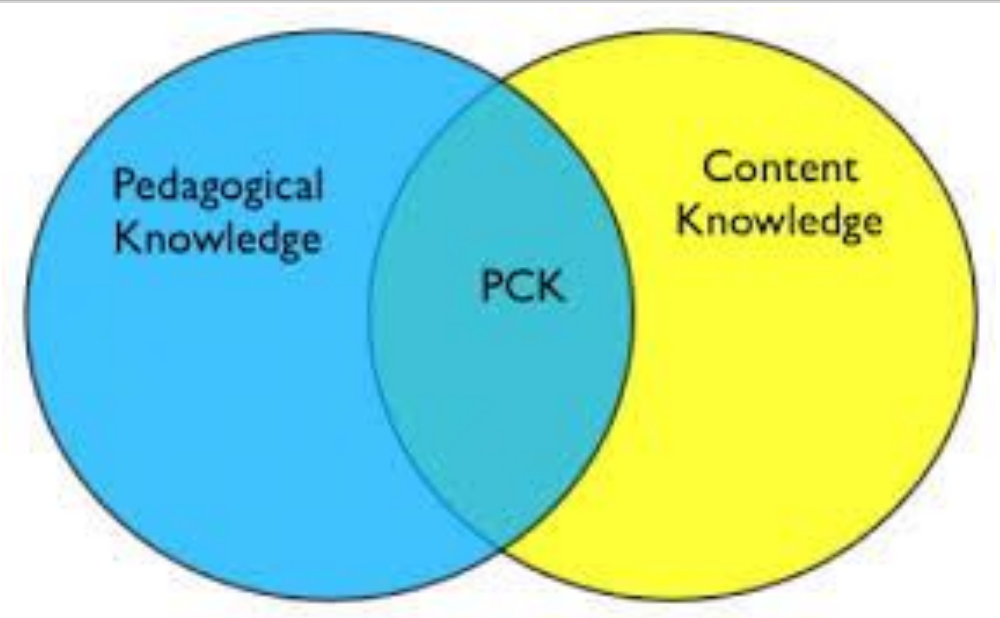

Figure 2.3. Shulman's (1986; 1987) Pedagogical Content Knowledge diagram illustrates both the blending of pedagogy and content, as well as their independence.

Pedagogy. Teachers' development of student learning experiences occurs during the pedagogical process of teaching. While teachers must understand various theories about how students learn, equally as important, is the selection of the best pedagogy for teaching students. The development of learning activities in a constructivist environment begins with a teacher's pedagogy. The Oxford Desk Dictionary and Thesaurus (1997) defines pedagogy as the "science of teaching" (p. 581). Merriam-Webster Online (2014) provides the definition as "the art, science, or profession of teaching." This science of teaching prompts teachers to think carefully about the best practices and most effective methods to reach students and offer them meaningful ways to interpret and apply the content. Simon (1995) discusses pedagogy and states that it is "meant to signify all contributions to the mathematical education of students in mathematics classrooms" (p. 114). He further says that pedagogy includes "the contributions to classroom learning of curriculum designers, educational 
materials developers, and educational researchers" (p. 114). Pedagogy would include research-based, effective instructional strategies.

Effective teaching. Pedagogical practice includes effective instruction and teachers must foster this active role by offering students opportunities for learning that builds on their prior knowledge (Staub \& Stern, 2002). The teacher's role in the education process has been researched and debated. James Coleman (1966) suggested in his study that the classroom teachers had little impact on student achievement and that other socio-economic factors demonstrated a larger effect. Yet, Hunter and Schmidt (1990), as well as Rosenthal (1991) argued that Coleman's (1966) data set demonstrated effective instruction resulted in an increase in academic growth. Hunter and Schmidt (1990) and Rosenthal's (1991) argument has been a foundation for educational reform. Many variables cannot be controlled when students walk into a classroom, but effective instructional strategies demonstrated by a teacher can be controlled.

The level of quality instruction a student receives is determined and dictated by teachers and administrators (Marzano et al., 2001). Marzano and colleagues (2001) meta-analysis or synthesis of empirical educational research revealed a list of nine effective research-based instructional strategies that positively impact student achievement. These nine research-based strategies are:

- Identifying similarities and differences

- Summarizing and note taking

- Reinforcing effort and providing recognition

- Assigning homework and practice 
- Using nonlinguistic representations

- Cooperative learning

- Setting Objectives and providing feedback

- Generating and testing hypotheses

- Questions, Cues, and advance organizers (Marzano, Pickering, \& Pollock, 2001, p.7)

Through the meta-analysis of educational research on instructional strategies, Table 2.1 illustrates how these strategies have been found to have significant effect sizes on the learning of concepts. According to Shavelson (1996) an effect size of 0.5 or greater is shown to demonstrate a medium to significant effect. Each strategy in the table is listed in rank order from the highest effect size to the smallest effect size. The most researched teaching strategy and the one with the highest effect size of 1.61 is having students identify similarities and differences. The least effective strategy examined in the study, was the use of questions, cues, and advanced organizers with an effect size of .59 but this still represents a medium to significant effect. Using these nine strategies can help promote concept retention (Marzano et al., 2001). As teachers prepare for effective mathematics instruction, the use of these strategies would be beneficial to incorporate into their pedagogy. 
Table 2.1 Nine-Effective Teaching Strategies

Category/Strategy

Average Effect Size

1.) Identifying

similarities and

differences

2.) Summarizing and

1.00

note taking

3.) Reinforcing effort

.80

and providing

recognition

4.) Assigning homework

and practice

5.) Using nonlinguistic

representations

6.) Cooperative learning

7.) Setting objectives

and providing feedback

testing hypotheses

9.) Questions, cues, and advance organizers.

Note. The strategies are identified in Marzano, Pickering, and Pollock's

(2001) meta-analysis and text "Classroom Instruction that Works: ResearchBased Strategies For Increasing Student Achievement".

In addition to effective teaching strategies, the faculty at the University of Michigan School of Education (2015), are currently researching the importance of high-leverage practices found in teaching. These high-leverage practices are being evaluated in realistic learning labs at the university. They are working with 
Educational Testing Services to help design measures that will evaluate the effectiveness of these practices using computer simulations that replicate a real classroom (University of Michigan, 2015). Currently, they have identified 19 highleverage practices as shown in Table 2.2. 
Table 2.2. High-Leverage Teaching Practices from the University of Michigan

1.) Making content explicit through explanation, modeling, representations, and examples.

2. Leading a whole-class discussion.

3.) Eliciting and interpreting individual students' thinking.

4.) Establishing norms and routines for classroom discourse central to the subject-matter domain.

5.) Recognizing particular common patterns of student thinking a subjectmatter domain.

6.) Identifying and implementing an instructional response to common patterns of student thinking.

7.) Teaching a lesson or segment of instruction.

8.) Implementing organizational routines, procedures, and strategies to support a learning environment.

9.) Setting up and managing small group work.
10.) Engaging in strategic relationshipbuilding conversations with students.

11.) Setting long-and short-term goals for students referenced to external benchmarks.

12.) Appraising, choosing, and modifying tasks and texts for a specific learning goal.

13.) Designing a sequence of lessons toward a specific learning goal.

14.) Selecting and using particular methods to check understanding and monitor student learning.

15.) Composing, selecting, interpreting, and using information from methods of summative assessment.

16.) Providing oral and written feedback to students on their work.

17.) Communicating about a student with a parent or guardian.

18.) Analyzing instruction for the purpose of improving it.

19.) Communicating with other professionals.

Note. These high-leverage practices are currently being researched to measure their effectiveness on teaching (University of Michigan, 2015). 
Teachers who also incorporate digital technology into their instruction would have the flexibility to easily incorporate several effective strategies and practices into instruction. Through the tap or click of a button students could receive a digital similarity and differences task in which they would create a digital diagram through software such as Kidspiration (2015). Some technologies, such as GoClass (2015), also allow teachers to monitor student work immediately through the use of a handheld tablet. This type of technology allows teachers to use formative assessment to provide quick feedback to reinforce effort and recognize achievement. In addition, by monitoring students progress on a screen teachers can better support students who have acquired the conceptual knowledge, as well as quickly respond and address student misconceptions while they are occurring. Digital technology, such as interactive applications, can reduce and possibly eliminate the wait time for student feedback. Coupling the highly effective teaching strategies with the use of digital technology could better support student understanding of mathematical concepts.

To further promote effective instructional strategies and mathematics understanding the Common Core State Standards (NGA Center, 2010) has outlined a set of eight mathematical practices to help facilitate mathematical proficiency. Table 2.3. identifies these mathematical practices in the CCSSM (NGA Center, 2010). These practices help to guide educators to provide experiences that impact student learning and achievement in all areas of mathematics. Applying these practices to the instruction of fractions in this study will help students to conceptualize their understanding and add deep meaning to the skills (Chval et al., 2015). 
Table 2.3. Common Core Standards Mathematical Practices

\begin{tabular}{|c|c|}
\hline Practice & Explanation \\
\hline MP1 Mathematical Practice 1 & $\begin{array}{l}\text { Make sense of problems and } \\
\text { persevere in solving them. }\end{array}$ \\
\hline MP2 Mathematical Practice 2 & $\begin{array}{l}\text { Reason abstractly and } \\
\text { quantitatively }\end{array}$ \\
\hline MP3 Mathematical Practice 3 & $\begin{array}{l}\text { Construct viable arguments and } \\
\text { critique the reasoning of others. }\end{array}$ \\
\hline MP4 Mathematical Practice 4 & Model with mathematics. \\
\hline MP5 Mathematical practice 5 & $\begin{array}{l}\text { Use appropriate tools } \\
\text { strategically }\end{array}$ \\
\hline MP6 Mathematical Practice 6 & Attend to precision. \\
\hline MP7 Mathematical practice 7 & $\begin{array}{l}\text { Look for and make use of } \\
\text { structure. }\end{array}$ \\
\hline MP8 Mathematical practice 8 & $\begin{array}{l}\text { Look for and express regularity } \\
\text { in repeated reasoning. }\end{array}$ \\
\hline
\end{tabular}

Note. Adapted from "Common Core Standards For Mathematical Practice", by National Governors Association Center for Best Practices, Council of Chief State School Officers. Copyright 2010 by the National Governors Association Center for Best Practices, Council of Chief State School Officers, Washington D.C. 
The CCSS (NGA Center, 2010) the standards for mathematical practices offer a viable approach to incorporate Marzano and colleagues (2001) research based strategies into mathematics instruction. The strategies and practices align giving a cohesive, effective approach to mathematical understanding. For example, the nine research based strategies outlined in Marzano and colleagues (2001) reveal that generating and testing hypothesis and using nonlinguistic representations demonstrate high effect sizes. These instructional strategies align with CCSM Mathematical Practices MP3. Construct viable arguments and critique the reasoning of others and MP4 Model with mathematics. Students should have ample opportunities to come to their own conclusions about mathematical problem solution strategies. Through estimation and making hypotheses students construct an argument about a particular problem. Then through the use of various mathematical models students are able to determine whether their arguments are viable. These practices also encourage constructivist learning, as students are not only constructing arguments but also justifying their thinking in solving particular problems.

Marzano and colleagues (2001) found that identifying similarities and differences had a high effect size of 1.61. In research on fractions this strategy can be seen in Fosnot and Dolk's (2002) case study example of students sharing sandwiches. The students were presented with a problem in which they had to determine how to effectively and equally share sandwiches on their museum field trip. Students drew models of the sandwich shares for the trip. Students then discussed the differences and worked through the problem collaboratively. Students in this case study were able to use fraction models to compare and contrast the differences in fraction sizes. 
In addition, students created viable arguments and critiqued the reasoning of other through the collaborative problem solving effort.

Teachers should have sound pedagogical knowledge that promotes the CCSS (NGA Center, 2010) standards for mathematical practices as well as the nine research based strategies presented in Marzano and colleagues (2001) meta-analysis on effective teaching. Teachers' understanding of learning theories and effective, research based teaching strategies helps promote mathematical concept retention in students (Fosnot \& Dolk, 2002; Marzano et al., 2001; NGA Center, 2010; Shulman, 1986). This mathematical concept retention promotes student mathematical proficiency and helps support later work with more sophisticated or complex mathematical concepts.

Content knowledge. In addition to pedagogical practice, teachers must also have knowledge of the content they are teaching. Hill, Rowan, and Ball (2005) determined that, "teachers mathematical knowledge for teaching positively predicted student gains in mathematics achievement during first and third grades" (p. 399). In their study of 115 elementary schools they examined teacher surveys and student achievement data to determine the impact of teacher content knowledge on student achievement, with special attention to student achievement in mathematics. They found that in one model of their study "students gained roughly two and a quarter points on the Terra Nova for every standard deviation difference in teachers' mathematics content knowledge" (p. 396). In addition, they found that "teachers' mathematics preparation (i.e., the average number of content and methods courses they completed in preservice or graduate training) positively predicted student gains 
in the third grade but was just outside of traditional significance ( $p=.06)$ " (p. 398).

Hill, Rowan, and Ball's (2005) study provides evidence that teacher content knowledge does impact student mathematics performance.

Curriculum. Teachers have to make important decisions about how to teach and deliver the mathematics content. One-way teachers deliver content is through the use of published curriculum. Prior to the CCSSM (NGA, 2010) most American curriculums were published with many mathematical topics in a textbook in order to appeal to multiple state and local textbook adoption committees (Schmidt, Houang, \& Cogan, 2001). This variation of skills and standards in a textbook led to a wide array of topics. The discussion among current educators is the how this varied approach brings about a curriculum that is a mile-wide, and an inch-deep and often covers a broad range of content with limited depth (Schmidt et al, 2001). This description depicts the idea that each school year many key skills and standards are taught and retaught with American curriculums. However, the use of this approach may lead teachers to skim through concepts in order to introduce students to the many skills and never teach any one in-depth (Schmidt et al., 2001). This skim-over approach can have the effect of leaving students with major gaps and misconceptions in their understanding (Schmidt et al, 2001).

With a majority of the states adopting the CCSSM (NGA Center, 2010) textbook companies and curriculum developers are beginning to narrow the focus to cover fewer standards per grade level. CCSSM (NGA Center, 2010) document has five domains for grades three, four, and five. These domains include:

- operations and algebraic thinking 
- number and operations-base-ten

- number and operations-fractions

- measurement and data

- geometry (NGA Center, 2010)

The narrowing of standards per grade should allow teachers more time to teach topics in depth, and in turn promote stronger conceptual development for students that can result in fewer gaps in understanding.

Fractions. For the current study, the domain Number and OperationsFraction strand is a content focus in grades three through five. Fractions must be defined and explored in relation to student understanding. Meaningful experiences and contexts help to support student understanding in Number and OperationsFractions.

In order to understand fractions, the rational number system must first be identified as a new system and different from the whole number system. Rational numbers are an extension of the whole number system and are defined as a quotient of two integers (Barnett-Clarke et al., 2010; Battista, 2012). Elementary school students in grades three through five commonly work with rational numbers in two representations, (1) fractions and (2) decimals. Barnett-Clarke and colleagues (2010) define a fraction as a "symbolic expression of the form $\frac{a}{b}$ representing the quotient of the quantities (provided, as always, that the divisor $b$ does not represent zero)" ( $\mathrm{p}$. 15). Fractions extend the whole number system and allow us to look at parts of numbers in relation to a determined whole (Battista, 2012; Barnett-Clarke et al., 2010). Four key areas in the foundational understanding of fractions cited in research 
include, thinking of a fraction as an actual number, representation/symbolic meaning of fractions, fractional relations to the whole (equivalency), and understanding benchmark numbers in relation to the defined unit (Chval et al., 2015; McNamara, \& Shaughnessy, 2010; Petit et al., 2010). Battista also (2012) outlines seven critical components of understanding fractions:

- Partitioning.

- Fractions.

- Iteration.

- Equivalent fractions, comparisons, and operations.

- Fraction Sets.

- Fractions on number lines.

- Making the unit explicit. (Battista, 2012, pp. 1-9).

These essential skills help students to reason about fractions.

The CCSSM (NGA Center, 2010) recognizes these foundations suggested by Battista (2012) as important understandings in fractions. To illustrate this foundational understanding, Confrey and colleagues (2012) created a trajectory of the mathematical skills students navigate as they move through the grades. These seven skills are seen throughout the grade level trajectories. Confrey and colleagues worked with both the writers of the CCSSM (NGA Center, 2010) and researchers to help unpack the standards to help teachers discern when and how to teach each standard. This research model identifies eighteen learning trajectories for fractional concepts from kindergarten through eighth grade (Confrey, Maloney, \& Corley, 2014). 
Confrey et al. (2014) structured each trajectory in sections based on key concepts in fractions.

Students have informal experiences with fractions in the equipartitioning trajectory. Confrey et al. (2014) structured the equipartitioning trajectory as having only one section, "Equipartitioning evenly divisible collections" (Equipartitioning Section). This section identifies each key skill and standard for equipartitioning that is addressed in this trajectory, as shown in Table 2.4.

Confrey et al. (2014) structured the formal fractions trajectory as having three sections. These sections include:

- Section 1. Working with unit fractions.

- Section 2. Equivalence and comparisons of fractions.

- Section 3. Operations with fractions (Confrey et al. 2014, Fractions).

These sections identify each key skill and standard for formal fractions instruction that is addressed in this trajectory, as shown in Table 2.4.

Multiplication and division of fractions are addressed in the multiplication and division trajectory. This trajectory includes the multiplication and division of whole numbers as well as fractions. Multiplication and division of fractions are addressed in this trajectory. Five sections are in the multiplication and division trajectory and fractions are structured in these two sections:

- Section 3. Equipartitioning multiple wholes

- Section 5. Multiplication and division problems involving non-whole rational number operators (Confrey et al. 2014, Multiplication and Division). 
These sections identify each key skill and standard for the multiplication and division of fractions as seen in Table 2.4.

Table 2.4. CCSSM (NGA Center, 2010) Mathematical Standards for Fractions and the Trajectories

\begin{tabular}{|c|c|c|c|}
\hline Grade & Indicator & Descriptor & Trajectory Section \\
\hline \multirow[t]{2}{*}{$\begin{array}{l}3^{\text {rd }} \text { Grade } \\
\text { Standards }\end{array}$} & 3.NF.1 & $\begin{array}{l}\text { : fraction } \frac{1}{b} \text { as the quantity formed by } 1 \\
\text { part when a whole is partitioned into } b \\
\text { equal parts; understand a fraction } a / b \text { as } \\
\text { the quantity formed by parts of size } \frac{1}{b}\end{array}$ & $\begin{array}{l}\text { Equipartitioning: } \\
\text { Section } 1\end{array}$ \\
\hline & 3.NF.2 & $\begin{array}{l}\text { : Understand a fraction as a number on } \\
\text { the number line; represent fractions on a } \\
\text { number diagram. } \\
\text { A: Represent fraction } \frac{1}{b} \text { on a } \\
\text { number line diagram by defining } \\
\text { the interval from } 0 \text { to } 1 \text { as the } \\
\text { whole and partitioning it into } b \\
\text { equal parts. Recognize that each } \\
\text { part has size } \frac{1}{b} \text { and that the } \\
\text { endpoint of the part based at } 0 \\
\text { locates the number } \frac{1}{b} \text { on the } \\
\text { number line. } \\
\text { B: Represent a fraction } \frac{a}{b} \text { on a } \\
\text { number line diagram by marking } \\
\text { off a length } \frac{1}{b} \text { from } 0 . \text { Recognize } \\
\text { that the resulting interval has a } \\
\text { size } \frac{a}{b} \text { and that its endpoint locates } \\
\text { the number } \frac{a}{b} \text { on the number line. }\end{array}$ & $\begin{array}{l}\text { Fractions: } \\
\text { Section } 1\end{array}$ \\
\hline \multirow[t]{2}{*}{$\begin{array}{l}4^{\text {th }} \text { Grade } \\
\text { Standards }\end{array}$} & 4.NF.1 & $\begin{array}{l}\text { : Explain why a fraction } \frac{a}{b} \text { is equivalent } \\
\text { to a fraction }(n X a) / n X b) \text { by using } \\
\text { visual fraction models with attention to } \\
\text { how the number and size of the parts } \\
\text { differ even though the two fractions } \\
\text { themselves are the same size. Use this } \\
\text { principle to recognize and generate } \\
\text { equivalent fractions. }\end{array}$ & $\begin{array}{l}\text { Fractions: } \\
\text { Section } 2\end{array}$ \\
\hline & 4.NF.2 & $\begin{array}{l}\text { : Compare two fractions with different } \\
\text { numerators and different denominators, } \\
\text { e.g. by creating common denominators or } \\
\text { numerators or by comparing to a }\end{array}$ & $\begin{array}{l}\text { Fractions: } \\
\text { Section } 2\end{array}$ \\
\hline
\end{tabular}




\begin{tabular}{|c|c|c|c|}
\hline & & $\begin{array}{l}\text { benchmark fraction such as } \frac{1}{2} \text {. Recognize } \\
\text { that comparisons are valid only when the } \\
\text { two fractions refer to the same whole. } \\
\text { Record the results of comparisons with } \\
\text { symbols and justify the conclusions e.g. } \\
\text { by using a visual fraction model. }\end{array}$ & \\
\hline \multirow[t]{2}{*}{$\begin{array}{l}5^{\text {th }} \text { Grade } \\
\text { Standards }\end{array}$} & 5.NF.3 & $\begin{array}{l}\text { : Interpret a fraction as division of whole } \\
\text { numbers leading to answers in the form } \\
\text { of fractions or mixed numbers. }\end{array}$ & $\begin{array}{l}\text { Multiplication } \\
\text { and } \\
\text { Division: } \\
\text { Section } 3\end{array}$ \\
\hline & 5.NF.4 & $\begin{array}{l}\text { : Apply and extend previous } \\
\text { understandings of multiplication to } \\
\text { multiply a fraction or whole number by a } \\
\text { fraction. } \\
\qquad \text { A: Interpret the product }\left(\frac{a}{b}\right) \times q \\
\text { as a parts of a partition of q into } \\
\text { equal parts; equivalently, as the } \\
\text { result of sequence of operations } a \\
\quad \times \frac{q}{b}\end{array}$ & $\begin{array}{l}\text { Multiplication } \\
\text { And } \\
\text { Division: } \\
\text { Section } 5\end{array}$ \\
\hline
\end{tabular}

Note. This table shows the mathematical standards set forth by the CCSSM (2010) and their relationship to Confrey et al. (2014) mathematical learning trajectories.

Confrey, Maloney, Corely (2014) spent time specifically looking at research pertaining to equipartitioning as they see equipartitioning as the foundation for more advanced fractional concepts. Battista (2012) states, "To understand fractions, students must be able to partition a whole into equal portions and understand how the portions are related to the whole" (p. 2). Kamii \& Clark (1995) found in their work with 120 fifth and sixth grade students, that $44 \%$ of fifth graders and $51 \%$ of sixth graders were able to accurately demonstrate that they recognized a "half." The learning trajectories for the K-8 common core math standards (Confrey et al., 2012) suggests that students should have sufficient practice with equipartitioning prior to moving forward on any other fractional concepts. 
Equipartitioning is often seen in primary grades as fair shares (Confrey, 1988). Students are given a problem in which they must divide some sort of object (candy bar, pie, pizza, brownies, cookies, etc.) amongst a determined number of people. Students work individually or as a group to make conjectures and test them to determine the fractional parts needed to solve the problem (Chval et al., 2013; Empson, 2001; McNamara, \& Shaughnessy, 2010). As students work together, they construct knowledge about partitioning areas or regions By giving students extensive experiences in this partitioning areas or regions McNamara and Shaughnessy (2010) suggests that this "can help them develop a deep and flexible understanding of partwhole relations" (pg. xv).

Developing Understanding in Fractions. Fractions are mathematical concepts that students struggle to fully understand (Barnett-Clarke et al., 2010; Kilpatrick et al., 2001). On the 2004 National Assessment of Educational Progress (NAEP), 50\% of eighth graders could not order three fractions from least to greatest (NCTM, 2007). This result according to Petit et al., (2010) could possibly be due to a teachers' lack of knowledge and ill-formed pedagogical practice. Other reasons include time constraints and district pressures resulting in teachers using a set of expired rules to teach mathematical concepts to students quickly (Asku, 1997; Karp, Bush, \& Dougherty, 2014; Mack, 1995; McNamara, \& Shaughnessy, 2010). Ill-formed pedagogical practices often leave children with low levels of conceptual understanding in fractions.

Students' understanding of fractions depends on both accommodations of effective conceptual development and procedural knowledge (Petit et al., 2010). 
Rittle-Johnson, Siegler, and Alibali (2010) have found, “Children's initial conceptual knowledge predicted improvements in procedural knowledge" (p. 357). They argue that conceptual understanding and procedural knowledge develop hand in hand. In their study on these two levels of understanding, they found causal evidence that conceptual understanding and procedural fluency influence one another. RittleJohnson, Siegler, and Alibali (2010) argue that by using multiple and continuous measures of both conceptual and procedural knowledge a teacher can effectively close proficiency gaps in understanding. They found that the development of conceptual understanding and procedural fluency supports Kilpatrick and colleagues' (2001) argument that proficiency should be developed collectively.

Proficiency in fractions is achieved through activities that are concrete, semiconcrete, to abstract (Heddens, 1964; Witzel, 2005). These three levels of instructional representations align with the learning trajectories for the K-8 Common Core Math Standards (Confrey et al., 2012). Offering students multiple representations of fractional concepts helps to build their concrete or conceptual understanding of a fraction. In the example of fair shares, where students are asked to share something equally, after students develop initial understanding of part-whole relationships teachers should vary numbers in the problems given. Presenting students a variety of activities in which wholes are partitioned in various ways helps them to identify and further strengthen the part-whole relationship (McNamara \& Shaughnessy, 2010). When students have sufficient practice with a fractional concepts they are then able to build upon the constructed idea and move from semiconcrete to abstract thinking. This concept of learning supports a constructivist 
learning style because students are using their experiences to build new ideas about a particular concept (Piaget, 1954; Petit et al., 2015). Learning contexts can help to facilitate sufficient understanding in fractions skills.

Contexts to support fraction understandings. Using the standards for mathematical practices and effective-research based strategies, two meaningful contexts emerge that can support students' understanding of fractions:

- Developing understanding of fractional relationships to whole numbers and unit representations.

- Extensive modeling of fractional concepts through area, length, and set models.

Fractions are represented in units. These unit representations demonstrate the part-whole relationship. Regional models, number lines, and sets of objects helps students to better visualize the part-whole relationship (Chval et al., 2013; Petit et al, 2010). Regional models include circular regions, tiles, and fractions strips. Giving students multiple opportunities to practice with various types of number lines helps them build fraction fluency and understanding of the whole in relation to the unit representation (Shaughnessy, 2011).

When working with fractions students should explore meaning through a variety of area, length, and set models (Chval et al., 2013; McNamara, \& Shaughnessy, 2010; Van de Walle, Karp, \& Bay-Williams, 2013). Many of the indicators in the Common Core State Standards (NGA Center, 2010) for Mathematics, ask students to justify their answers and as an example provide visual proof, e.g. visual models. In addition, the NCTM Process Standards requires students to "select and use various types of reasoning and methods of proof" (p. 4). It becomes 
essential for teachers to give students various types of experiences and models to assist in constructing their mathematical knowledge.

Students should not be limited to rules or tricks when solving mathematical problems. The teaching of rules with whole number operations can lead to confusion when introducing fractional concepts (Asku, 1997; Mack, 1995; McNamara, \& Shaughnessy, 2010). Researchers have found that students may inappropriately apply their knowledge and understanding of whole numbers to fractional concepts.

For example, Mack (1995) found in her independent case studies of seven third and fourth grade students, that students use their knowledge and strategies of whole numbers to make sense of fractions. She cited a case study example, Greg, stating that:

See, I know this other sort of math [fractions], but I know this [whole numbers] better, so I try to make it so it looks like just a regular math problem so I can figure out the answer. It's the same thing. It's just easier to figure out this way (p. 437). Mack's (1995) study demonstrates the importance of ensuring students have constructed their knowledge about fractions by demonstrating their relationships to whole numbers and by using models.

In another study, McNamara and Shaughnessy (2009) also found that students struggled with fractional concepts because of poorly understood teacher-taught fractions rules. In one example, students had been taught that the smaller the denominator the larger the fraction. 
Figure 2.4. Case Example of Fraction Rules

$$
\text { Circle the larger fraction } \frac{5}{6} \quad \frac{7}{8}
$$

Explain your answer

Figure 2.x. As shown in Beyond Pizzas and Pies: 10 Essential Strategies for

Supporting Fraction Sense (p. 4) (McNamara, \& Shaughnessy, 2009).

When asked the question in Figure 2.4. students cited the larger fraction was $\frac{5}{6}$ based on the idea that this fraction contained the smaller denominator.

Students should have opportunities to construct their knowledge and understanding of mathematical concepts, including fractions through the use of effective-research based strategies and practices (Marzano et al., 2001; NGA Center, 2010). These opportunities go beyond the teaching of mathematical rules. Asku (1997) found that "fraction rules can easily become the focus of rote learning and produce artificial feelings of accomplishment" (p. 375). He found in his study of 155 sixth grade students that, in the computation portion of the assessment, students had similar performance ratings between those who had higher conceptual development tasks than those who did not prior to the assessment. However, in the same sample, students who had greater conceptual development of fractional concepts had greater results in problem solving than those who had only used rote rules. This provides evidence of the importance of guiding students in developing mathematical ideas conceptually utilizing effective-research based strategies and practices.

Student Progress and Proficiency. Kilpatrick and colleagues (2001) have integrated five strands of understanding that define student proficiency in 
mathematics as shown in Table 2.5. Each of these strands "capture what we think it means for anyone to learn mathematics successfully" (p. 5). 
Table 2.5. Mathematical Proficiency

\begin{tabular}{ll}
\hline \multicolumn{1}{c}{ Proficiency } & \multicolumn{1}{c}{ Explanation } \\
\hline Conceptual Understanding & Comprehension of mathematical \\
& concepts, operations, and \\
& relations \\
& Skill in carrying out procedures \\
Procedural Fluency & flexibly, accurately, efficiently, \\
& and appropriately \\
& Ability to formulate, represent, \\
and solve mathematical & problems \\
Capacity for logical thought, \\
reflection, explanation, and \\
justification \\
Habitual inclination to see \\
mathematics as sensible, useful, \\
and worthwhile, coupled with a \\
belief in diligence and one's \\
own efficacy. \\
Productive Disposition & \\
&
\end{tabular}

Note. Demonstrates the five strands of mathematical proficiency discussed in Adding It Up: Helping Children Learn Mathematics (Kilpatrick, Swafford, \& Findell, 2001, p. 5). 
Students grow and develop on a learning continuum, meaning that proficiency is not finite (Confrey et al., 2012). While the five strands are interwoven, students may be at different levels on any strand at any given time. The CCSS (NGA Center, 2010) Standards for Mathematical Practices help to develop these proficiencies in students' mathematical understanding. Table 2.6 shows the relationship between both the proficiency strands and mathematical practices. 
Table 2.6. Relationship between proficiency strands and CCSS Mathematical Practices

Proficiency Strand

Conceptual Understanding

Procedural Fluency

Strategic Competence

Adaptive Reasoning

Productive Disposition

\section{Mathematical Practice}

- MP.1 Make sense of problems and persevere in solving them.

- MP.2 Reason abstractly.

- MP.5 Use appropriate tools

- MP.3 Construct viable arguments.

- MP.4 Model with mathematics.

- MP.8 Look for and express regularity in solving problems.

- MP.3 Construct viable arguments.

- MP.7 Look for and make use of structure.

- MP.8 Look for and express regularity in solving problems.

- MP.2 Reason abstractly.

- MP.1 Make sense of problems and persevere in solving them.

Note. The CCSSM Mathematical practices (CCSSO, 2010) help to support Kilpatrick, Swafford, and Find ells' (2001) five strands of mathematical proficiencies.

Student exploration of fraction contexts should happen within their core mathematics instructional program. Core mathematics instruction is the primary environment students receive mathematics instruction, developing their mathematical concept retention, cultivating their confidence, and supporting strong proficient 
mathematical thinking (Buffum, Mattos, \& Weber, 2009). In the core mathematics instruction classroom a highly qualified teacher helps oversee student progress. This teacher designs effective lessons to help students learn and meet standards of proficiency.

In a multi-tiered system of support that is founded on effective core mathematics instruction, $80 \%$ or more of students are meeting standards with proficiency as monitored through progress monitoring system (Gersten, \& NewmanGonchar, 2011). Progress monitoring is aligned with the standards to measure precisely students' performance on the standard that has been taught and the effectiveness of instruction (Gersten, \& Newman-Gonchar, 2011). Progress monitoring can be determined through a series of both formative and summative assessments. Using this monitoring system assists teachers in determining which students have achieved mastery of the core standards. Effective teaching and progress monitoring in the core mathematics instructional program plays a critical role in narrowing the gap of students' understanding.

\section{Technological Pedagogical Content Knowledge (TPACK)}

Pedagogical Content Knowledge (Shulman, 1986; 1987) integrates two main areas as central components of the teaching process. Koehler and Mishra (2008) propose that the use of technology also plays a critical role. Their framework, technological, pedagogical, content knowledge (TPACK), integrates technology into the teaching process. Technology should integrate into pedagogical practice and content knowledge so that learning activities engage students in the learning process (Mishra \& Koehler, 2006). Figure 2.5 illustrates how technology interacts with 
content and pedagogical knowledge. A goal of this study is to explore and apply the TPACK framework when designing mathematics lessons with the purpose of increasing student proficiency in fractions.

Figure 2.5. TPACK Framework

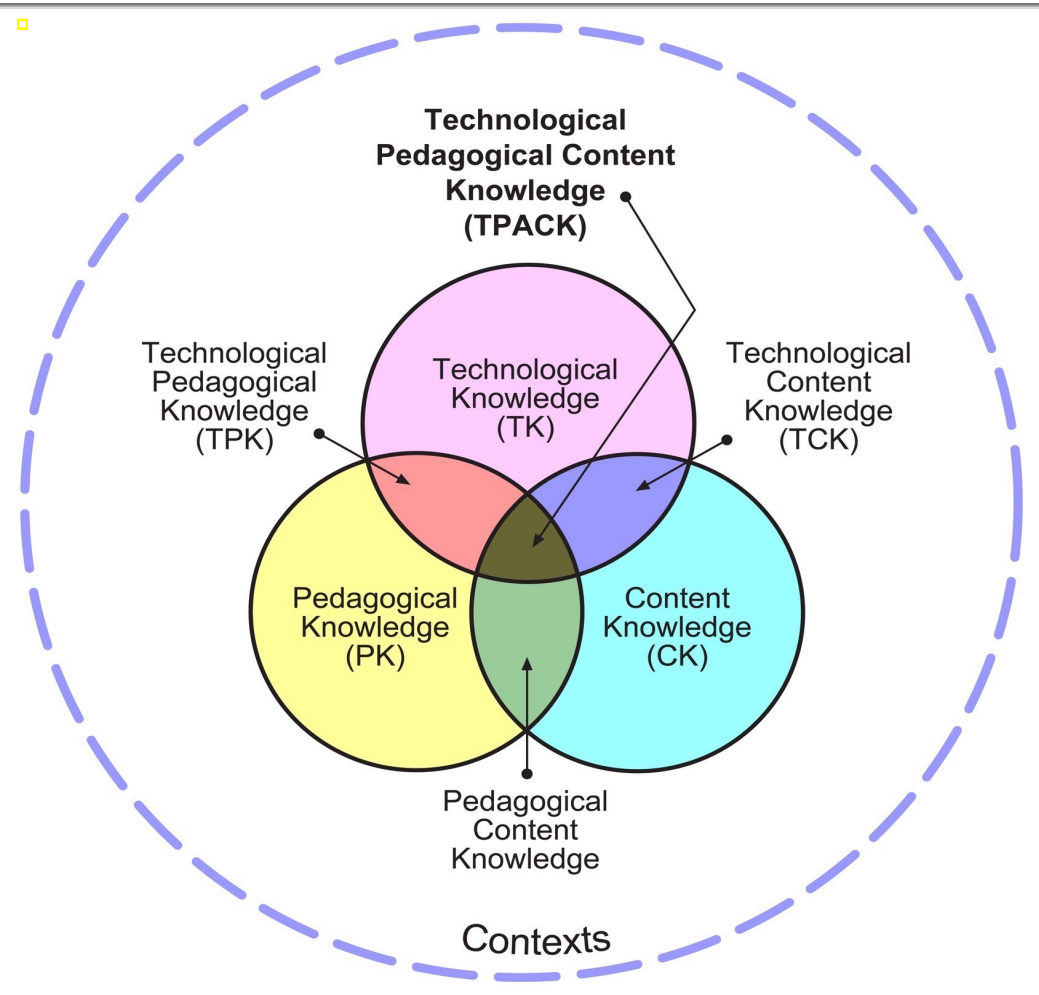

Figure 2.5. Mishra and Koehler's graphic representation of the TPACK framework.

(http://tpack.org, 2006)

Importance of technology integration. The CCSS (NGA Center, 2010) has identified the importance of using technology in the classroom. After elementary school, students are required to specifically use technology to demonstrate their mathematical understanding. For example, in grade 7 students are asked to draw 
geometric shapes with given conditions using technology (NGA Center, 2010). The important statements issued about technology should set the precedent for classroom teachers to integrate technology into mathematics instruction starting in the elementary grades.

The National Council for Teachers of Mathematics (NCTM) issued a statement with regards to technology in 2008. Technology is an "essential tool for learning mathematics in the $21^{\text {st }}$ century" (NCTM, 2008, p. 1). NCTM (2000) specifically states that all students have access to computers and graphing calculators. However, teachers can expand their technology integration to include computer-based devices such as digital microscopes, digital cameras, telecommunication tools, email, internet web sites, multimedia software, electronic probe ware, CDs, software applications, spreadsheets, electronic calculators and web page authoring software (Irving \& Bell, 2004).

Teachers and technology. The simplicity of integrating technological tools and guiding students to build learning connections lies within the teacher's technology knowledge and students' access (Reinhart et al., 2011). Technology should also be used in ways that promotes constructive learning and higher order thinking skills (Morphew, 2012; Reinhart et al., 2011). One goal of the current study is for students to develop conceptual understanding, which requires higher order thinking skills that go beyond memorization (Kilpatrick et al., 2001).

In Reinhart et al.'s (2011) study of teachers enrolled in education and technology classes they found that socio-economic factors impact the facilitation of 
technology using higher-order thinking skills. Teachers in lower income schools have more support when using technology than in higher income schools. Using a survey with a $64 \%$ response rate, Reinhart et al. (2011) determined that "schools with a lower percentage of students who received free and reduced lunch were more likely to have a technology facilitator" (p. 187). The role of the technology facilitator was described as someone who is to provide in house support to teachers in the inclusion of technology in classroom activities. Reinhart et al.'s (2011) study “suggests that there is a link between the presence of a technology facilitator and the students using higher order thinking skills" (p. 189). Their conclusions support the need for "training and support from technology facilitators aids teachers with using technology to promote higher order thinking" (p. 190). Therefore, teachers who broaden their understanding of technology integration and receive supported are better equipped to implement technology in their classrooms that will promote students' higher order thinking and in turn higher proficiency. For the current study, the researcher aides as a technology facilitator in the treatment group. As the technology facilitator, she worked with the teachers to implement the use of an interactive mathematics application that promotes conceptual understanding and the use of higher order thinking skills.

As part of their knowledge base, teachers who want to integrate technology into their instruction must have an understanding of the Technological Pedagogical Content Knowledge (TPACK) framework (Koehler \& Mishra, 2008). Teachers must work toward integrating technologies into the pedagogical process. The way that teachers integrate technologies in their pedagogical process should extend learning to 
help students build and construct new knowledge (Jonassen, 2000).

\section{Summary}

This chapter addressed the literature that supports the conceptual framework for this study. The reviews included four primary topics, constructivist learning theory, pedagogy, content knowledge, and TPACK. These topics provide central ideas in support of the teaching and learning of mathematics.

\section{Restatement of the Research Question}

1.) How will elementary student achievement be impacted by the use of an interactive tablet application designed to facilitate student construction of responses to fraction problems? 


\section{CHAPTER III}

\section{METHODOLOGY}

\section{Rationale}

The purpose of this study is to examine the impact of an interactive tablet application, designed to facilitate student construction of responses to fraction problems on student achievement in fractions. As cited in Petit, Laird, and Marsden's (2010) book and as part of the On-going Assessment Project (OGAP) researchers

found in a study of Vermont $4^{\text {th }}$ graders that about " 44 percent $\left(\frac{133}{308}\right)$ of all incorrect responses analyzed on the OGAP pre-assessment were attributed to the use of inappropriate whole number reasoning (VMP OGAP, (2005). [Grade 4 preassessment]. Unpublished raw data)" (pp. 31-32). This lack of fractional understanding between whole numbers and fractions has important impacts on learning future mathematical topics such as algebra (Barnett-Clarke et al., 2010). While this is a disturbing trend in students' performance in the topic of fractions, this study adds to the current field of research by demonstrating that the integration of technology can booster student achievement in fractions.

\section{Research question}

How will elementary student achievement be impacted by the use of an interactive tablet application designed to facilitate student construction of responses to fraction problems? 


\section{Population}

The population for this study is elementary students in grades three through

five. The sample of the population for this study is two groups within two elementary schools in the southwest portion of a large urban mid-western school district. These groups were sampled based on the researchers' access and proximity to the two schools in which the groups are located. The schools are within two miles of each other, with similar demographics as shown in Table 3.1 and Table 3.2.

Table 3.1. Enrollment of Population and Percentage of Free and Reduced Lunch

\begin{tabular}{|c|c|c|c|c|}
\hline & \multicolumn{2}{|c|}{ ENROLLMENT } & \multicolumn{2}{|c|}{ \% FREE/REDUCED LUNCH } \\
\hline & School $A$ & School B & School $A$ & School B \\
\hline 2013-2014 & 424 & 440 & 75.6 & 80.0 \\
\hline $2012-2013$ & 473 & 429 & 72.4 & 74.9 \\
\hline 2011-2012 & 489 & 454 & 74.6 & 73.1 \\
\hline
\end{tabular}


Table 3.2. Demographic Information of Sampled Schools.

\begin{tabular}{ccccccccc}
\hline & \multicolumn{2}{c}{ WHITE } & \multicolumn{3}{c}{ A. AMERICAN } & HISPANIC & \multicolumn{2}{c}{ OTHER } \\
\cline { 2 - 8 } & School & School & School & School & School & School & School & School \\
& $A$ & $B$ & $A$ & $B$ & $A$ & $B$ & $A$ & $B$ \\
$13 / 14$ & 60.6 & 44.8 & 30.0 & 41.6 & 2.6 & 7.0 & 6.8 & 6.6 \\
$12 / 13$ & 56.2 & 43.1 & 35.9 & 43.1 & 2.3 & 3.5 & 5.5 & 4.9 \\
$11 / 12$ & 52.1 & 40.3 & 40.9 & 40.3 & 2.9 & 3.5 & 4.1 & 3.1 \\
\hline
\end{tabular}

Note. This table represents the demographic information of sampled schools. Data were retrieved from the district website, but is undisclosed to remain anonymous.

Table 3.1 shows the enrollment and percentage of free and reduced lunch at each sampled school. The schools are located in similar the same geographic, carry similar numbers of enrolled students, and have similar proportions of students with free and reduced lunch rates. Table 3.2 shows the overall demographic make-up of the sampled schools. School A represents the treatment group and School B represents the control group.

Sample. The sample for this study is a convenience sample. The researcher has built relationships at both schools and with the principals. Both principals are interested in contributing to research that results in student achievement growth. This study examines a digital approach to instruction as a way to increase student achievement growth in fractions.

The sample consists of students who are in grades three through five at School A and School B. The assignment of the treatment group was non-random and was done as a convenience. Students in grades three through five at School A serve as the 
treatment group. Students in grades three through five at School B serve as the control group. Table 3.3 represents the number of classes and students in each group. Table 3.3. Classes and students in each group.

\begin{tabular}{ccccc}
\hline Grade Level & \multicolumn{2}{c}{ Number of Classes } & & \multicolumn{2}{c}{ Number of Students } \\
Third & $\begin{array}{c}\text { Group A } \\
\text { (Treatment) }\end{array}$ & $\begin{array}{c}\text { Group B } \\
\text { (Control) }\end{array}$ & $\begin{array}{c}\text { Group A } \\
\text { (Treatment) }\end{array}$ & $\begin{array}{c}\text { Group B } \\
\text { (Control) }\end{array}$ \\
Fourth & 3 & 3 & 65 & 61 \\
Fifth & 2 & 2 & 44 & 42 \\
Total & 7 & 2 & 43 & 42 \\
\hline
\end{tabular}

Note. This table represents the number of students in both treatment and control groups.

Grades three through five were selected for several reasons. Foremost, these grades are cited in Confrey and colleagues' (2012) Hexagon Map of Learning Trajectories for Common Core State Standards in Mathematics as being the first formal level in which students learn fractions. Second, student struggles in fractions are reflected at a national level. Local district assessments also reflect a worrisome trend in student achievement in fractions.

Results from the Seventh Mathematics Assessment (2000) highlighted results of the 1996 NAEP assessment. Results indicated that only $50 \%$ of fourth graders can "indicate the number of fourths in a whole." Only $31 \%$ of fourth graders could "indicate the placement of a fraction $\frac{n}{d}$ on a unit number line divided in multiple of $d$ parts." As part of the OGAP in Vermont, researchers found that about 59\% students 
would order the fractions $\frac{1}{3}$ and $\frac{1}{4}$ right after $\frac{1}{2}$ on a number line (Petit et al., 2010). This indicates that students apply their whole number reasoning to fractions when looking at the denominator, as in counting whole numbers when 3 and 4 follow 2. Latest district data also shows students' lack of fractional understanding in similar areas as seen in Table 3.4. 
Table 3.4 District data on assessed fractions skills

\begin{tabular}{|c|c|c|c|}
\hline \multirow{2}{*}{$\frac{\text { District Assessed }}{\text { Fractions Skills }}$} & \multirow[t]{2}{*}{ Grade } & \multicolumn{2}{|c|}{ Percentage Correct } \\
\hline & & Treatment & Control \\
\hline $\begin{array}{l}\text { Understanding Unit } \\
\text { Fractions. }\end{array}$ & $3^{\text {rd }}$ & $78 \%$ & $90 \%$ \\
\hline $\begin{array}{l}\text { Comparing } \\
\text { Fractions. }\end{array}$ & $4^{\text {th }}$ & $78 \%$ & $52 \%$ \\
\hline $\begin{array}{l}\text { Decomposing and } \\
\text { composing fractions } \\
\text { for addition and } \\
\text { subtraction. }\end{array}$ & $4^{\text {th }}$ & $55 \%$ & $64 \%$ \\
\hline $\begin{array}{l}\text { Developing the } \\
\text { concept of dividing } \\
\text { unit fractions. }\end{array}$ & $5^{\text {th }}$ & $51 \%$ & $40 \%$ \\
\hline $\begin{array}{l}\text { Interpreting } \\
\text { multiplying fractions } \\
\text { as scaling. }\end{array}$ & $5^{\text {th }}$ & $50 \%$ & $34 \%$ \\
\hline $\begin{array}{l}\text { Using equivalency to } \\
\text { add and subtract } \\
\text { fractions with unlike } \\
\text { denominators. }\end{array}$ & $5^{\text {th }}$ & $74 \%$ & $45 \%$ \\
\hline $\begin{array}{l}\text { Understanding the } \\
\text { concept of } \\
\text { multiplying fractions } \\
\text { by fractions. }\end{array}$ & $5^{\text {th }}$ & $54 \%$ & $53 \%$ \\
\hline
\end{tabular}

Note. These data were retrieved from district documents from the Data Management, Planning, and Program Evaluation (1/8/2015).

The district monitors student proficiency through their developed mathematics proficiency assessments three times a year. Table 3.4 shows the district report filed as of January 2015 based on student scores on the district mathematics proficiency assessments. District assessed skills are based on the Common Core State Standards, 
but are adjusted to fit the curriculum maps developed by the district. The percentages in Table 3.4 represent the number of correct answers students demonstrated on district proficiency assessment (Data Management, Planning, and Program Evaluation, 2015). These assessments were only used to demonstrate the current level of students' fractional understanding. By targeting grades three through five this study may increase overall student achievement in fractions content.

This district has developed a curriculum-pacing guide for all levels of mathematics instruction. Mathematics content is covered in a period of four cycles in grades three through five. Each cycle has varying lengths depending on the content covered and fractions are taught along with other mathematical concepts. Table 3.5 illustrates the tentative/ expected cycle length in days that fractions content is covered. This length is dependent on teachers' pacing of instruction and student achievement as determined through assessment. This study occurred over a ten-week period during the cycles listed. The cycles created through the district are created to last around nine-weeks. An observation period of ten-weeks allowed for any disruptions of inclement weather days, assemblies, or other classroom disruptions. 
Table 3.5 District pacing for content

\begin{tabular}{ccc}
\hline Grade Level & Cycle & $\frac{\text { Number of Expected Days }}{\frac{\text { Required to Teach }}{47 \text { Days }}}$ \\
Third & Cycle 3 & 45 Days \\
Fourth & Cycle 2 & 44 days \\
Fifth & Cycle 2
\end{tabular}

Note. This table illustrates the cycle in which fractions are taught in each grade level and the estimated length of that cycle.

Treatment. Many mathematics applications exist for tablet devices. The goal of the researcher was to find an application that would provide an open platform with flexibility for uploaded and flexible in the types of activities available for students. The GoClass (2011) application offered the flexibility for users to upload their selfcreated or pre-selected content and activities.

GoClass is a web-based application offered for iOS and Android operated devices. GoClass will operate on tablet devices such as iPad (2014), iPhone (2014), and iTouch (2014). The application is available through a free download on Apple's App store. GoClass will also operate on android operated devices such as Nexus 7 and Chromebook. and available through download on Google Play store. One can access GoClass directly through a computer or laptop. GoClass has two sides, one that controls the loaded content on the web and the application that users interact with the web-loaded content on a tablet or sign-on through a computer. The web-based side of GoClass allows instructors to control the desired content, student rosters, 
questioning results, and scheduling of whole-group sessions and individual instructional sessions.

The procedures for this study occurred during normal core mathematics instruction in the classroom. As discussed in an earlier chapter, core mathematics instruction includes whole group teaching, small group instruction, math workshop, and other methods appropriate for delivering math content. All students in grades three through five at both schools participate in the normal mathematics classroom.

Treatment was delivered during math workshop. Math workshop is a time during normal mathematics instruction that gives teachers the opportunity to work with small groups of students to develop and help them make progress with their mathematical skills. Math workshop occurs two to three times per week most of the school year with exception of schedule interruptions (i.e. assemblies, inclement weather, etc.). Math workshop is part of common practice in the treatment and control schools. During math workshop, students who are not working directly with the teacher often participate in independent or small group activities for math practice. Often these independent activities or small group activities include math games and projects. When students are not working directly with a teacher the students in the treatment group worked on the GoClass application as part of their workshop time. Working on GoClass will be a replacement for working with paper and pencil activities. Students in the treatment group did not receive any additional mathematics instruction that the control group will not receive as well.

All treatment students in grades three, four, and five worked through ten independent instructional sessions on GoClass over the course of a ten-week period 
during the instructional cycle devoted to fractions. The researcher worked with the teachers in professional learning communities to develop the instructional sessions that were then loaded onto the GoClass application. Teachers helped the researcher to target specific fractional standards. Students can access the instructional sessions at any time with an Internet or data connection. These sessions allow students to work freely with the content without direct teacher guidance. The instructional sessions are designed to last no more than twenty-five minutes and allow students to work on their own pace. Students may access GoClass at home, however, the only requirement that exists in session completion is that students must complete them at school during math workshop. This was monitored by the application using the GoClass logs and reports function that tracks student logins. Students were not likely to complete more than one session a day. Teachers in the treatment only have math workshop two to three days a week. Therefore, it was determined that a ten-week time period would provide ample time for students to complete ten sessions.

During the instructional GoClass sessions, students will engage in a series of applications and other digital activities to help them build knowledge about fractions content. Applications will be used as tools to develop concepts, and not used for rotepractice or remediation (Jonassen, 2000). Digital applications have built in controls to keep access to only the targeted programs.

Students in the treatment group will complete the instructional sessions on a tablet device. Student work created through the tablet will be submitted electronically for scoring. Work submitted through GoClass will serve as a fidelity measure for the purpose of the study. GoClass reports can be downloaded into an 
Excel worksheet and show who has completed each session, the time required for completion, and the date of completion. This report will serve as evidence that the lessons were completed with fidelity. The classroom teachers will have an option to use the digital work for a grade or an assessment measure of student progress.

Lesson Development. For the purpose of this study the instructional sessions were designed collaboratively with the classroom teachers, but each were loaded under the researchers' username and password on the GoClass application. Loading classes and lessons under the researchers' username and password will help to make the process uniform. Teachers will be responsible to sign up for designated times through the ten weeks to have access to the iPad (2014) cart. Teachers will also have one twenty-minute session during team planning to view a brief overview of the application that will be used in the research. No daily or weekly trainings will transpire regarding the application. Third and fifth grade teachers have never previously used the GoClass application. The fourth grade mathematics teacher attended a three-hour professional development on the application offered through the school district and taught by the researcher. The teacher does not currently use the application in her instruction. The fourth grade teacher will receive a ten-minute review of the application. This introduction and review of the application are to help the teacher's field student questions if needed. While teachers worked to create the instructional sessions, teachers will not have to load any content on GoClass. Restricting the loading of lessons to the researcher will encourage the uniformity of implementation of the application. 
Lesson Overview. GoClass sessions are focused on fractions content and designed for students to work at their own pace are developed through a combination of teacher created elements on the application. An element is a term used by GoClass to specify each activity uploaded to GoClass. Each activity placed into an element is teacher created. Each element will have characteristics that help with concept development. The four lesson plan elements are designed to last twenty-five minutes, or one session by definition for the study. Table 3.6 describes the overall lesson layout. 
Table 3.6. Outline of Treatment GoClass Teacher Created Lessons

\begin{tabular}{|c|c|c|}
\hline & Title & Description \\
\hline 1.) & Introduction Video & $\begin{array}{l}\text { This video will introduce the } \\
\text { activity. A description of } \\
\text { needed external applications, } \\
\text { mathematics notebook, and } \\
\text { overall expectations will be } \\
\text { given for the lesson. This video } \\
\text { should not be longer than two to } \\
\text { three minutes. }\end{array}$ \\
\hline 2.) & "I can" Statement & $\begin{array}{l}\text { "I can" statements are an } \\
\text { integral part of the expected } \\
\text { educational practices within this } \\
\text { district. An "I can" statement is } \\
\text { the target skill or standard that a } \\
\text { student will be working on in a } \\
\text { given lesson. Teachers use the } \\
\text { "I can" statements offered in } \\
\text { district curriculum maps. }\end{array}$ \\
\hline 3.) & Problem Solving Activity & $\begin{array}{l}\text { Teachers will pose a question or } \\
\text { several problems that will } \\
\text { require students to construct an } \\
\text { answer. Students may construct } \\
\text { an answer with in the GoClass } \\
\text { application by using the } \\
\text { scribble function of the } \\
\text { program. The scribble function } \\
\text { allows students to write on a } \\
\text { blank part of the screen with } \\
\text { their finger or stylus. Students } \\
\text { may also be asked to explore an } \\
\text { external application on the iPad } \\
\text { such as Geoboard, Kidspiration, } \\
\text { or ABCya Virtual } \\
\text { Manipulatives. Students will } \\
\text { construct their knowledge by } \\
\text { digitally drawing or } \\
\text { manipulating objects within } \\
\text { these external applications. The } \\
\text { requirements of each element } \\
\text { will be detailed in the } \\
\text { introductory video. In addition } \\
\text { to constructing an answer } \\
\text { students will be asked to } \\
\text { explain their thinking. }\end{array}$ \\
\hline 4.) & Two multiple choice questions & Students will conclude the \\
\hline
\end{tabular}




\begin{tabular}{|l|l|}
\hline & session by answering two \\
questions. These questions will \\
be similar in nature to the \\
questions they had to construct \\
answers. The questions will \\
serve as a lesson closure or exit \\
slip for the session.
\end{tabular}

Note. Provides the lesson framework for each GoClass instructional session.

The screen shot in Figure 3.1 illustrates how the lesson outlines look inside the GoClass application. The first element in the sequence is the introduction video. Within each element students will find their "I can" statement and a problem solving activity.

Figure 3.1. Screen Shot GoClass Lesson Outline

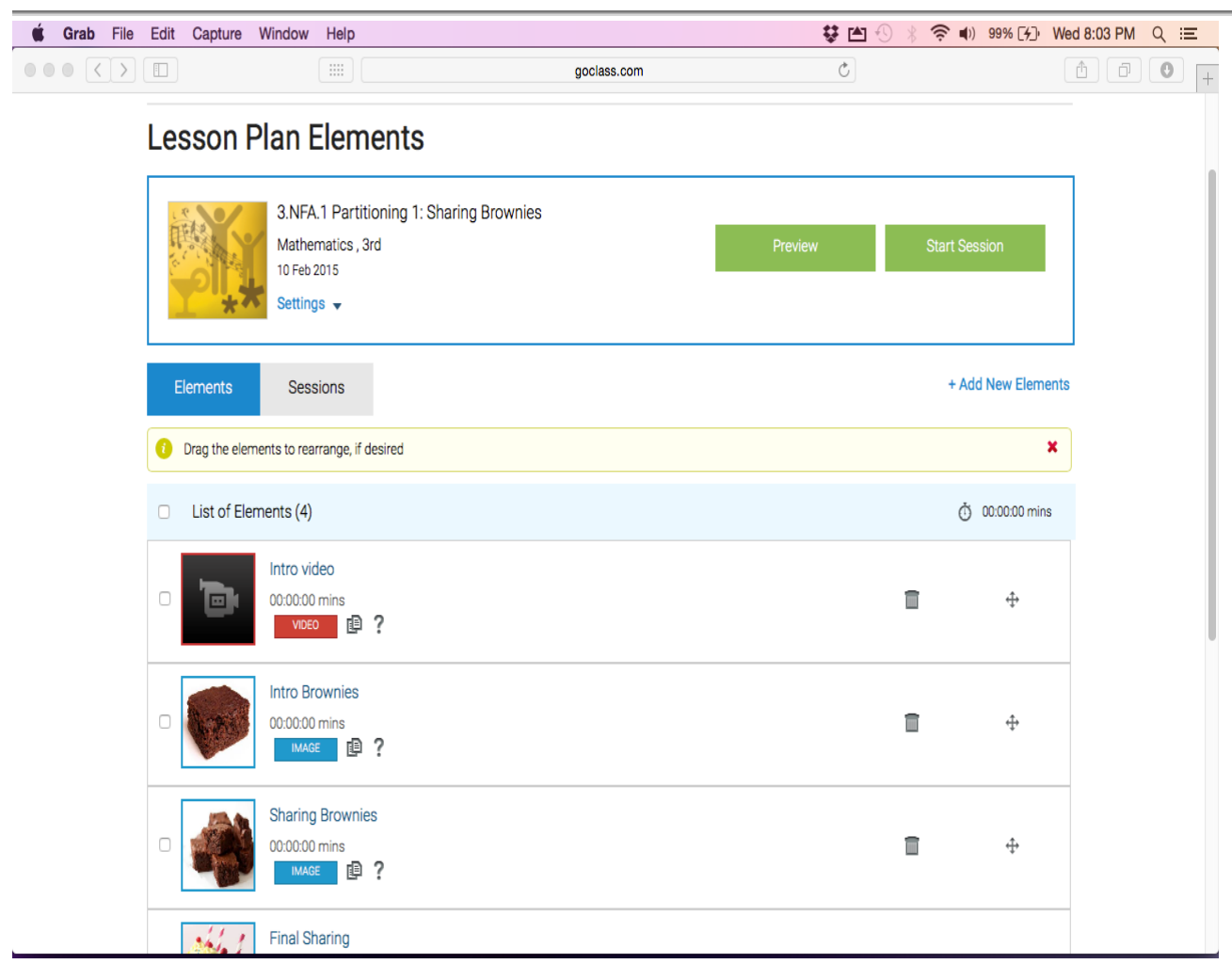

Figure 3.1. Example of what a teacher may see when looking at the elements of a instructional session done on the GoClass application. 
Within each individual GoClass elements are three parts, show, explain, and ask. For example, Figure 3.2 is an example of what students in third grade will see, a picture of a brownie in the "show" portion of this element.

Figure 3.2. Screen Shot GoClass "Show" Screen for Students

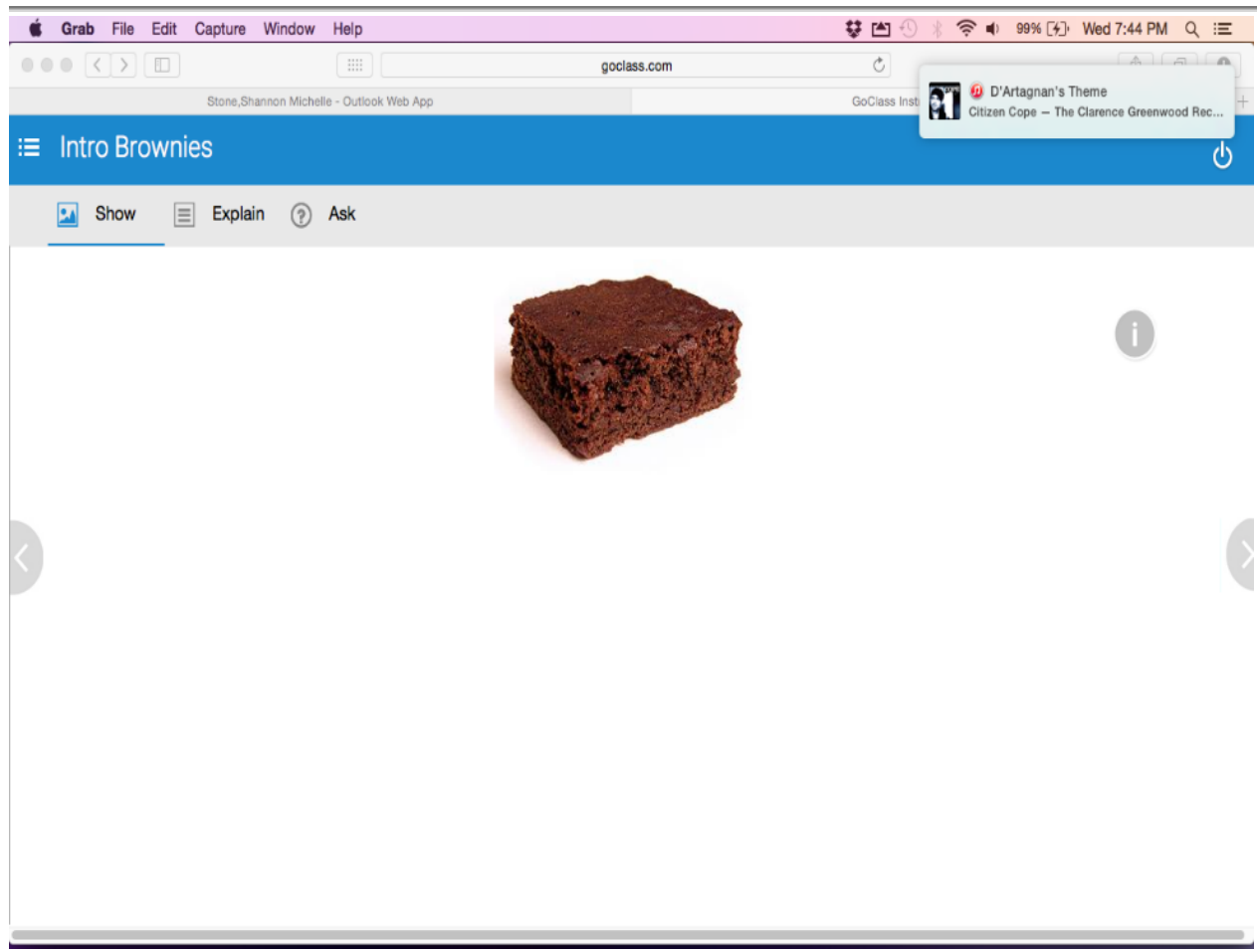

Figure 3.2. Shows an example what students may see in the "show" portion of a GoClass instructional session.

Students "I can" statement will be in the "explain" section of each element.

The screenshot in Figure 3.3 below is an example of what a students "I can" statement may look like within the GoClass application student view. 
Figure 3.3. Screen Shot GoClass "Explain" Screen for Students

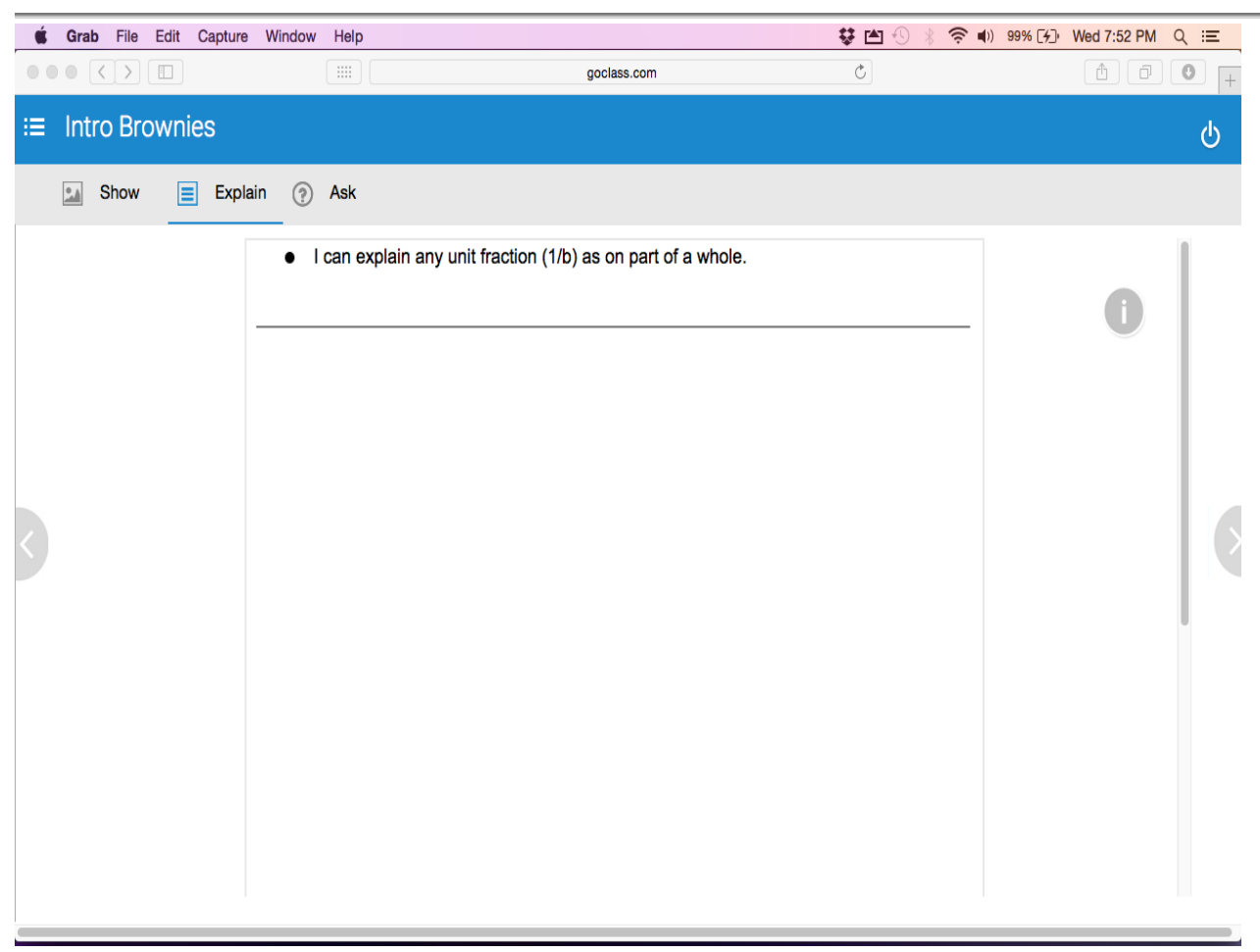

Figure 3.3. An example what students may see in the "explain" portion of a GoClass instructional session is shown.

In the "ask" portion of the element students are posed with a question or a series of questions. The teachers using mathematics resources such as their curriculum developed these questions. Students may use the scribble function to draw their answers. The scribble function of the application is a blank area that allows students to draw or write on the screen with their finger or with a stylus. Students will use the "Short Answer" function to explain their thinking by typing their answers. Students will use the "multiple-choice" function to answer multiple-choice questions. In some cases, to help build real-life connections students will answer a poll. Figure 3.4 shows a screenshot of a series of questions student will answer through 
explanation and scribble. These questions are only used for formative assessment of student understanding.

Figure 3.4 Screen Shot GoClass “Ask” Screen for Students

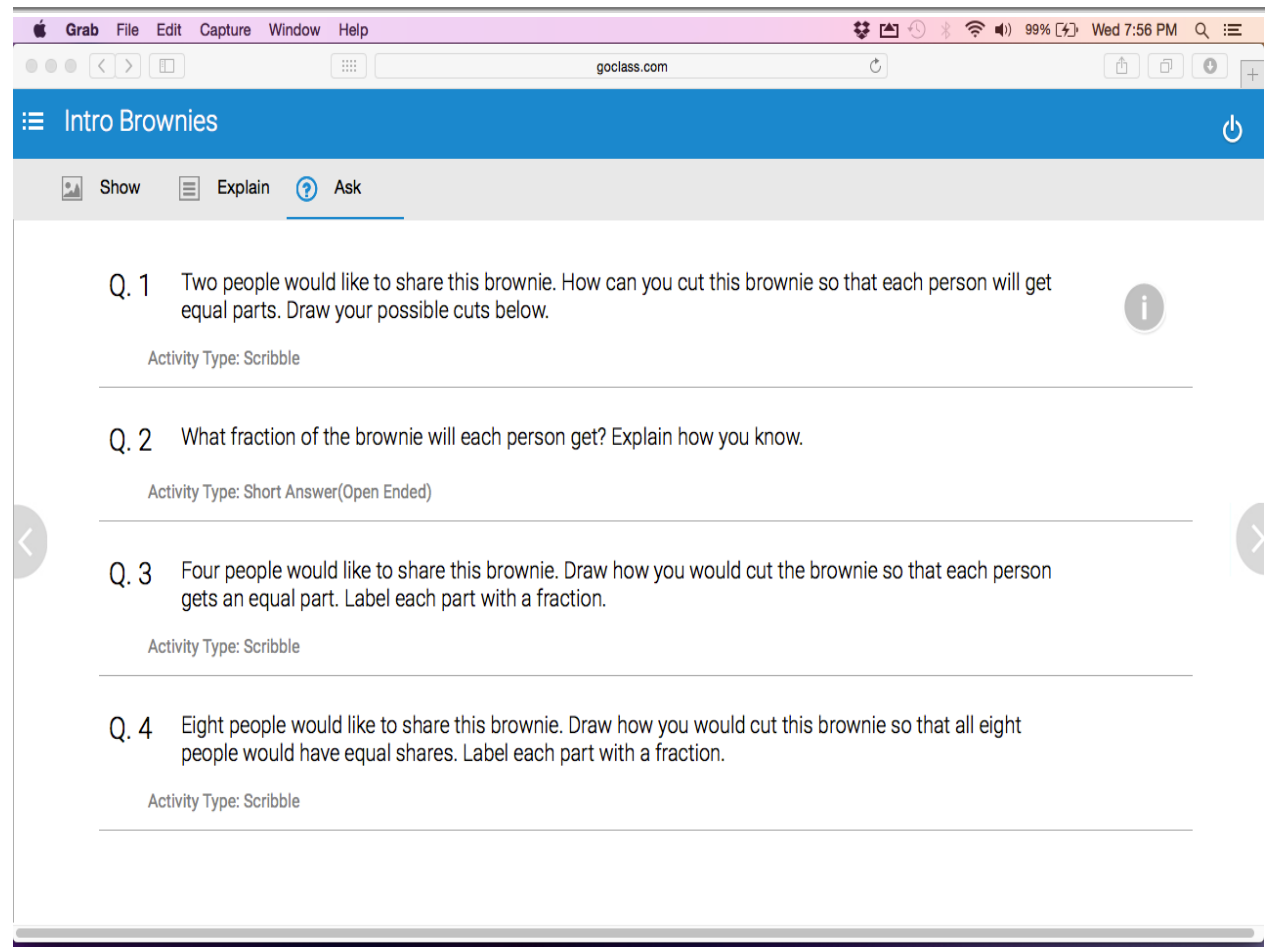

Figure 3.4. Shows an example what students may see in the "Ask" portion of a GoClass instructional session. 
Figure 3.5. Screen Shot GoClass Final Multiple-Choice Questions

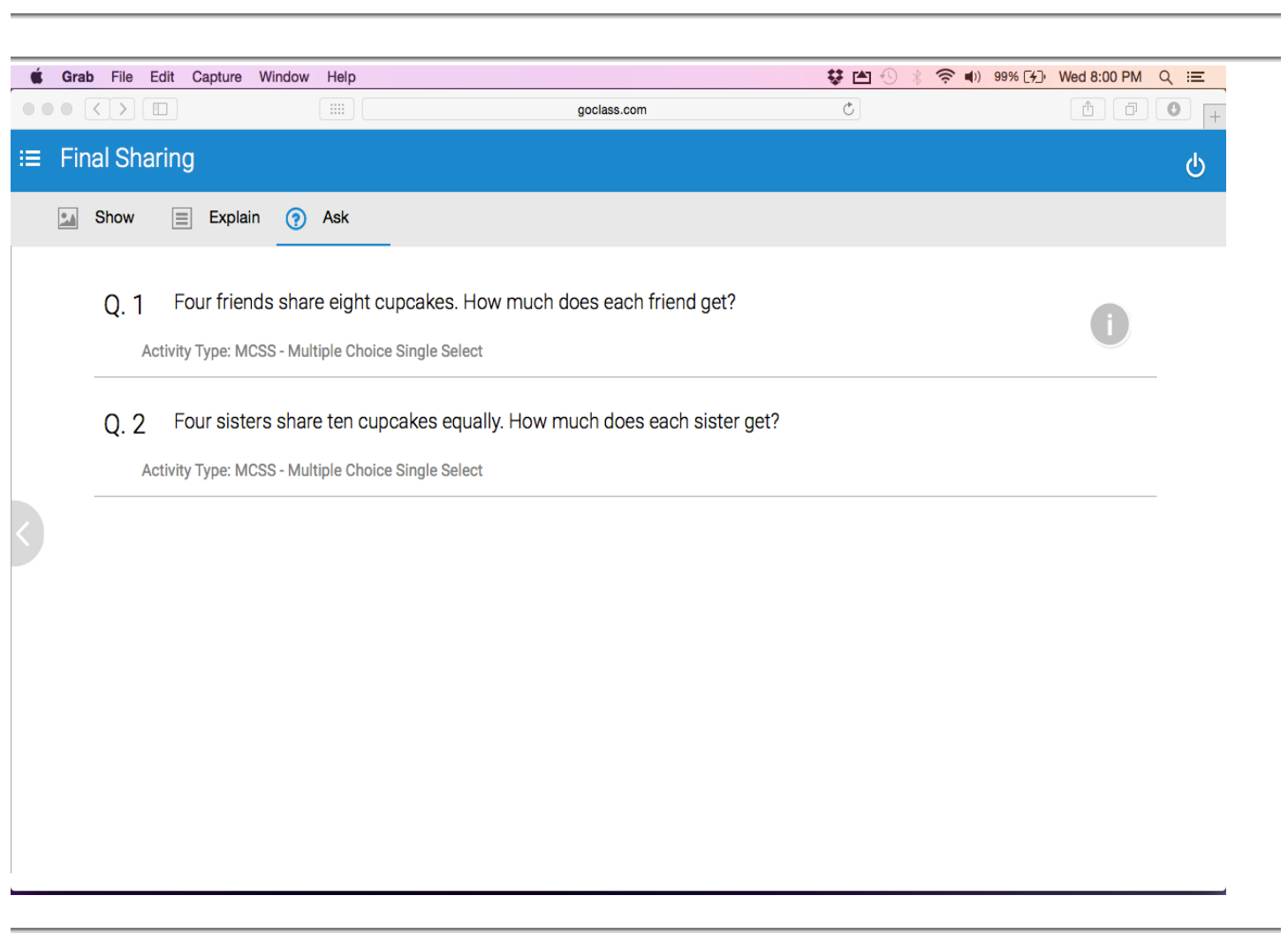

Figure 3.5. shows an example of what students may see for their final multiple-choice questions if they are part of their own GoClass lesson element.

Figure 3.5 shows examples of questions students may be presented with. Students will have the opportunity to use various interactive applications to help answer these questions. One example of an interactive application is Kidspiration that gives users graphic art they can use to represent problems or organize ideas. The application requires students to first construct the problem. Then they have to manipulate the graphic art characters in order to reach a solution. This manipulation requires students to think about the problem and the possible solution. By offering students the opportunity to explore the problem with graphic representations may help students 
construct new knowledge about partitioning. This newly constructed knowledge serves as the foundation for higher-level thinking about fractions. In Figure 3.6. this illustrates a way a student may construct Q.1. in Figure 3.5.

Figure 3.6. Kidspiration question set-up example
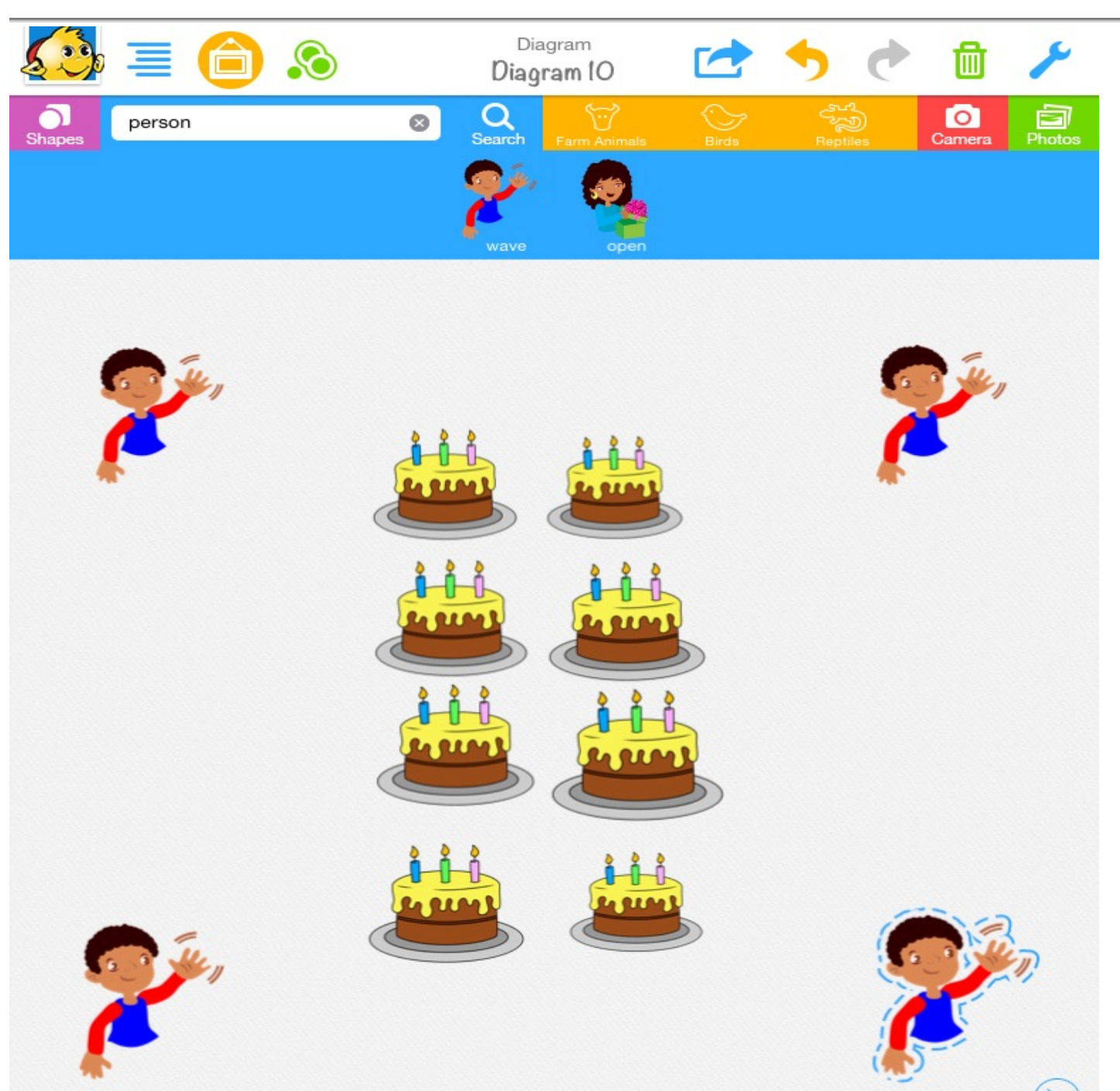

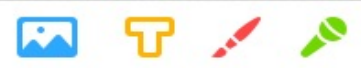

Figure 3.6. shows how students may use Kidspiration to set up the problem Q.1 given at the end of their GoClass session. 
In order to solve the problem, students can tap and drag items across the screen to their desired location. Figure 3.7. presents a possible graphic art answer to Q.1. in

Figure 3.6.

Figure 3.7. Kidspiration question answer example
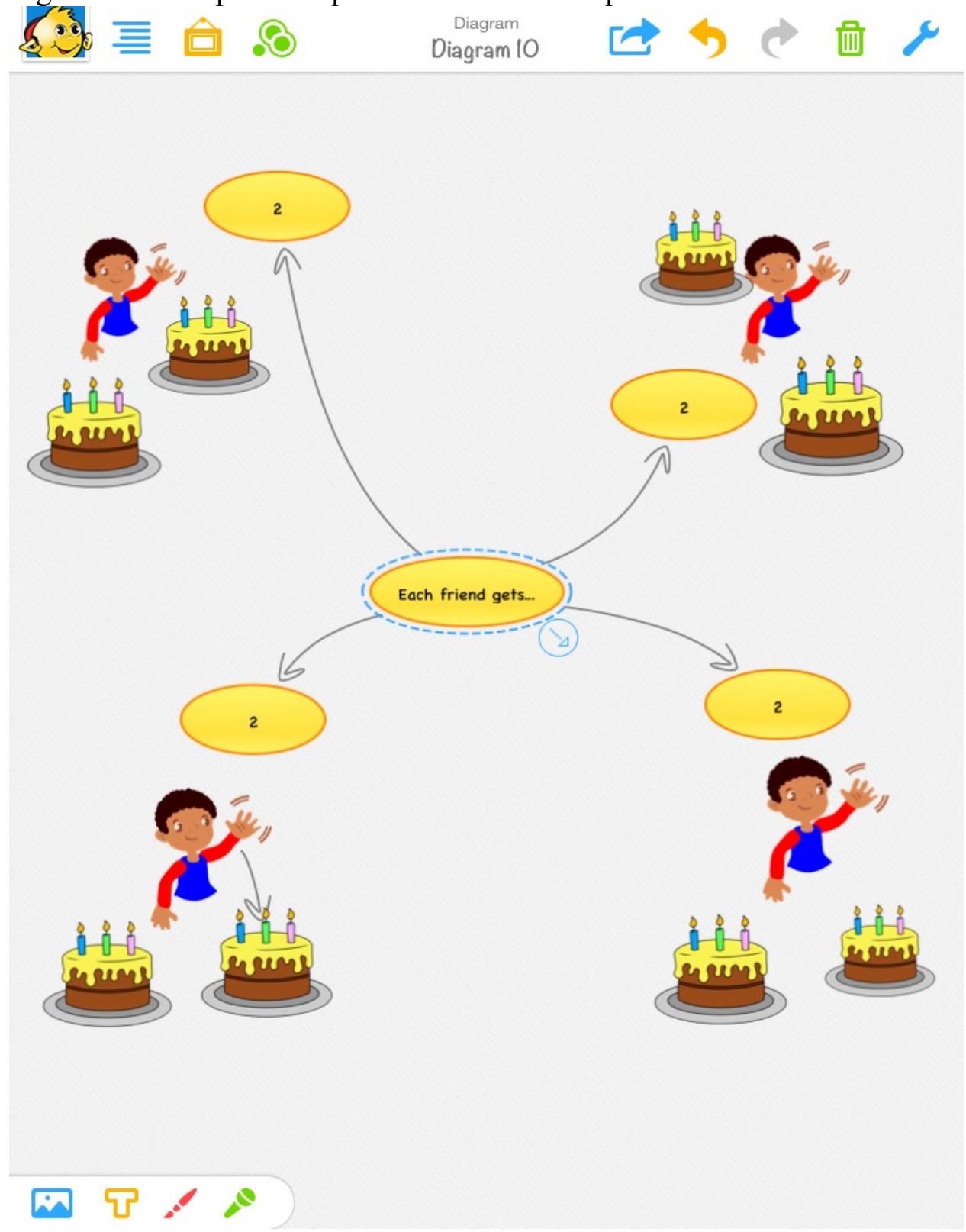

Figure 3.7. shows how students may manipulate the graphics on the tablet to help answer the question. 
Students can use applications such as Kidspiration to help them conceptually answer a question presented to them in GoClass. Students have to use technology tools available to them in the program to model the problem. To model the problem, students must know that there are eight cupcakes and four friends. Therefore, they have to drag eight cupcakes to the screen and four friends. Then students can tap and drag the cupcakes to each friend. This can also be solved using GoClass' scribble function. Students could choose to draw this picture out on grid paper. Rather than tapping and dragging the cupcakes, students would simply draw lines to the drawn friends. GoClass is flexible because students can construct multiple representations of the problem. Students may choose to use the Scribble function within the application or they may choose to go outside and use an application such as Kidspiration to help construct their knowledge about fractions. Using multiple representations may help student's construct stronger conceptual understanding about fractions.

The GoClass application is unique because it allows its users to develop answers by creating drawings and using other interactive applications, as opposed to answering strictly multiple response or short answer questions. GoClass offers the students the opportunity to visually represent their thinking to aide in the construction of new knowledge and deepen their conceptual development.

Student preparation to use GoClass. Prior to starting the treatment, students in Group A will engage in a thirty-minute session on the use of the technology in the GoClass (2011) application. During this session students will receive their login information and practice logging into the application on the iPad (2014). Students will interact with content briefly in the whole-group session function of GoClass 
(2014). In the whole group session the researcher will be the teacher and will control the content with which students interact. Students will then participate in two tenminute trial flip sessions on GoClass (2014) inside their mathematics classroom prior to starting any of the treatment ten instructional sessions. During this time students will be able to ask questions specifically of the researcher. The treatment third and fourth graders have not previously used the tablet-based application to the knowledge of the researcher. Fifth grade students who attended the same school in fourth grade used the tablet application in reading class during their fourth grade year. The fourth graders used the GoClass application six to seven times a month from September 2013 to April 2014. Those fifth graders are familiar with the login procedure and overall functionality of the application but have not previously used it for mathematics instruction.

Treatment Group. All treatment classrooms are at School A. Treatment groups will have core instruction and then have additional mathematics instruction on the topic of fractions that will include iPad applications as an interactive technology during mathematics workshop. This instruction will occur during the cycle noted in Table 3.5. The treatment school has a mobile set of forty iPads.

Control Group. All control classrooms are at School B. Control groups will participate in core classroom instruction the same as the treatment group. Control group students will also have additional mathematics instruction on the topic of fractions that will include additional center activities such as math games or projects the same as the treatment group. Math workshop occurs two to three days a week throughout the course of the school year, excluding normal classroom interruption 
(i.e. assemblies, inclement weather, etc.). All lessons during math workshop and whole group instruction will be created and delivered by the classroom teacher. During math workshop students will engage in math games, flashcards, small group instruction with a teacher, and project-based assignments (ordering from a restaurant menu, modifying a recipe, etc.). In place of the tablet application in the control group, students will engage in fractions learning during math workshop that will allow them to construct their answers to fraction problems using paper, markers, crayons, and manipulatives. The observation period of the control group will coincide with the cycle noted in Table 3.5.

Teachers in the control group may use some technologies in teaching, but will be limited to whole-class use and will not have the tablet technology based lessons in their math workshop time. For example, some teachers used interactive whiteboards to teach mathematics. Instruction occurred as normal, yet prohibiting the use of any extensive technologies in learning activities (e.g. iPads, laptops, netbooks, and other personal devices) during math workshop time. Typically the use of technology requires signing out from the site technology coordinator. The principal has agreed that any technology checked out during the observation period to a control teacher will be prohibited only during math instruction. The effort in the control group should be normal classroom instruction within those limits.

Students in the control group will participate in the same lesson goals as the treatment group, since both schools are using the district provided curriculum maps. Treatment and control groups will receive the same instruction. However, all work completed in the control group will be without the use of technology. 


\section{Research design}

This study is classified as quasi-experimental quantitative research.

Quantitative methods are defined by Teddlie and Tashakkori (2009) as the "techniques associated with the gathering, analysis and interpretation, and presentation of numerical information" (p. 5). This study collected information about students' performance on pre- and posttests using curriculum-based assessment, GoMath: Common Core Edition. In this study students will complete a pretest prior to any treatment. During the course of the study treatment and control teachers were observed to measure the classroom culture and environment during mathematics instruction. Students in both treatment and control groups will then complete a posttest to measure any treatment effects.

Shadish et al. (2002) describe quasi-experiments as “experiments” that lack random assignment of units to conditions but that otherwise have similar purposes and structural attributes to randomized experiments" (p. 104). More specifically this study is an embedded quasi-experimental design, as pre-test data will be collected prior to intervention. During the intervention period students will be observed in both treatment and control conditions. The researcher assigned school A to the treatment group. The researcher works at school A allowing her to monitor the implementation of treatment. School B was assigned control group because school B matched school A well and was close in proximity. 
This study was being conducted inside the regularly planned math workshop included in the classroom instructional day. Students continued with normal core content, following normal pacing guides and curriculum maps. Therefore, no deviance in content presentation occurred. However, the method of content delivery varied. Students in the treatment group worked with iPad applications to assist in mastering the fractions content during the math workshop period. Students in the control group continued with math journaling, projects, posters, or manipulatives without the use of individual technology applications in their instruction about fractions. Students in both groups are typically required to complete pre and posttests during the normal instructional course of a unit or lesson. Thus, no deviance in assessment procedures occurred during this study. Student participation is neither voluntary nor random.

In some studies that measure student achievement, researchers use quantitative methods. Quantitative methods are "a good example of an outcomesbased evaluation, where the emphasis is on whether a program has met its overall goals, typically measured quantitatively" (Teddlie \& Tashakkori, 2009, p. 9). In this study, student achievement is measured quantitatively. In the case of Marzano and Haystead's (2010) study on interactive whiteboards, student achievement was measured in a pre/posttest method and analyzed using quantitative methods. In Gano's (2011) study on Self-Selected Video (SSV) student achievement was also measured in a pre/ posttest method with an untreated control group design and analyzed using quantitative methods. The purpose of this study was to determine whether students who use tablet technology with the GoClass (2011) application will 
have higher levels of achievement on fractions content than a group using methods without tablet technology. The best way to measure this effect is through the determination of student achievement scores retrieved from student assessment in the regular education setting.

Additional quantitative methods were used to help determine the established mathematics classroom culture in both the treatment and control groups. After completing the pretest, teachers and students were observed using the Reformed Teaching Observation Protocol (RTOP) (Piburn \& Sawada, 2000). The RTOP has a reliability score of a .95 (Piburn \& Sawada, 2000). The RTOP rates important instructional qualities inside the classroom during the instructional period. These qualities look at students' prior knowledge, the overall lesson design, creativity, flexibility in problem solving, and the determination of fundamental concepts of the lesson. These qualities of instruction have impacts on student achievement (Marzano et al, 2001). Using the RTOP should help to control the impacts of instruction on both the treatment and control group. By controlling or explaining the qualities of instruction in the observed classrooms helped to explain any increases or significant differences in student achievement. By controlling for classroom instruction any differences in student achievement were attributed to the implementation of interactive technology using the GoClass (2014) application. 
Figure 3.8. Untreated Control Group Design with Dependent Pre-test, Posttest Samples

TECHNOLOGY

NO TECHNOLOGY

FIELD OBSERVATION
$\mathrm{PRE}_{1}$

$\mathrm{PRE}_{1}$

$\mathrm{O}_{1}$
$\mathrm{X}$

$\mathrm{POST}_{1}$

$\mathrm{POST}_{1}$

$\mathrm{O}_{2}$
$\mathrm{O}_{3}$

Note. Pre-tests using the GoMath curriculum will be given on fraction content. Posttests, also using the GoMath curriculum are given over the previously pre-tested then taught content. $\mathrm{X}$ is the technology instruction treatment, GoClass. $\mathrm{O}$ are observations done during the instruction period using the Reformed Teaching Observation Protocol (2010).

This study was expected to occur over a ten-week period during the spring 2015 semester. Figure 3.8 illustrates the research design of the study. The pre-test is given "because the groups are nonequivalent" (Shadish, Cook, \& Campbell, 2002, p. 138). The pretest helped to control for initial differences between the treatment and control groups. Table 3.7. presents the anticipated schedule for the research study. 
Table 3.7. Study Schedule

\begin{tabular}{|c|c|c|}
\hline Date(s) & Duration & Activity \\
\hline 2/9/15 & One-Time & $\begin{array}{l}\text { Pre-test given to treatment and control groups (third, } \\
\text { fourth, and fifth grades). }\end{array}$ \\
\hline $2 / 13 / 15$ & One-Time & $\begin{array}{l}\text { Completion of pre-test given to treatment and control } \\
\text { groups (third, fourth, and fifth grades). }\end{array}$ \\
\hline $\begin{array}{l}2 / 10- \\
2 / 13 / 15\end{array}$ & Three Days & $\begin{array}{l}\text { Treatment group given log-in ID's for the tablet } \\
\text { technology. Each group will practice logging-in to GoClass } \\
\text { and complete a practice activity. }\end{array}$ \\
\hline $\begin{array}{l}2 / 13 / 15- \\
2 / 20 / 15\end{array}$ & Seven Days & Grade and score all pre-tests. \\
\hline $\begin{array}{l}2 / 16- \\
4 / 30 / 15\end{array}$ & Ten-Weeks & $\begin{array}{l}\text { Ten-weeks are given to complete ten lessons on fractions } \\
\text { using the GoClass application. This ten-weeks accounts for } \\
\text { inclement weather and normal classroom disruptions (e.g. } \\
\text { assemblies, guest speakers, field trips). Teachers could } \\
\text { complete up to five sessions per week during math } \\
\text { workshop if they choose. }\end{array}$ \\
\hline $3 / 30-$ & Four & Treatment and control groups (third, fourth, and fifth \\
\hline $4 / 30 / 15$ & Weeks & $\begin{array}{l}\text { grade) teacher/classroom observations completed during } \\
\text { this five weeks. Each teacher of mathematics in these } \\
\text { groups will be observed three times during this time period. }\end{array}$ \\
\hline $5 / 4 / 15$ & One-Time & $\begin{array}{l}\text { Posttest given to treatment and control groups (third, } \\
\text { fourth, and fifth grades). }\end{array}$ \\
\hline $5 / 8 / 15$ & One-time & $\begin{array}{l}\text { Completion of posttest given to treatment and control } \\
\text { groups (third, fourth, and fifth grades). }\end{array}$ \\
\hline $\begin{array}{l}5 / 8- \\
5 / 15 / 15\end{array}$ & Seven Days & Grade and score all posttests. \\
\hline $\begin{array}{l}5 / 18- \\
5 / 29 / 15\end{array}$ & 14 Days & Enter all pre and posttest scores into an excel workbook. \\
\hline $\begin{array}{l}6 / 1- \\
6 / 5 / 15\end{array}$ & Five Days & Clean up missing data. \\
\hline $6 / 8-6 / 15$ & Seven Days & Run data in SPSS Software analyze output reports. \\
\hline
\end{tabular}

Note. This table represents the schedule outline for the completion of data collection and the analyzing of data. This schedule includes consideration for normal classroom disruptions (e.g. assemblies, guest speakers, field trips) and inclement weather disruptions. 
Variables. This study has three types of variables, independent, dependent, and a co-variate. The independent variable consists of two levels. While other variables only have one level.

Independent Variable. The independent variable (IV) in this study is classroom instruction. Classroom instruction has two categories, treatment, and control. In the treatment session students interacted with the GoClass application on iPad technology to participate in problem solving activities in math workshop. In the control session, students participated in the same type of problem solving activities using math games, projects, and practice activities. The control group used paper, pencil, markers, and crayons to complete their work in place of the GoClass application.

Dependent Variable. The dependent variable is the outcome of students' posttest scores on curriculum-based assessments on the topic of fractions.

Co-Variate. The co-variate in this study is students' pretest scores on curriculum-based assessment on the topic of fractions.

Power Analysis. A statistical power analysis was completed using the software program $G^{*}$ Power (2009). This power analysis determined that with six groups ( 2 classrooms at each of grades three, four and five) a minimum sample size of 162 participants would have adequate power with an effect size of .4, alpha level at .05 , power at .95 . Figure 3.9 . is the power analysis table conducted on G*Power (2009). The total sample size of this study should be more than 162 participants. 
Figure 3.9. Power Analyses

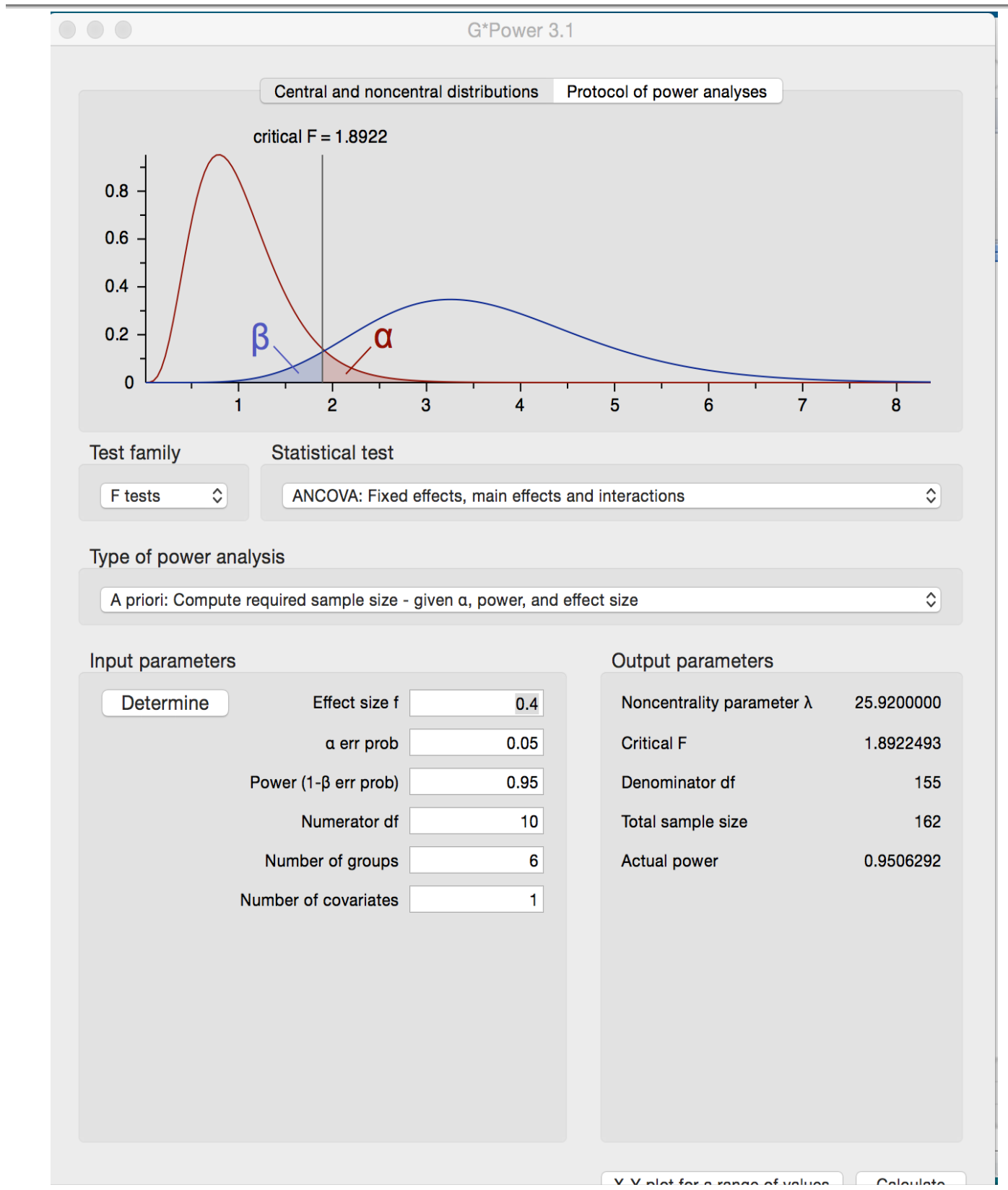

Figure 3.9. shows a screen shot of $\mathrm{G}^{*}$ Power (2009) power analysis completed for this study.

The researcher anticipated the sample size as $n=297$. Each third grade classroom in both groups has a maximum of twenty-four students and three third 
grade classrooms. Each fourth grade classroom in both groups has a maximum of twenty-eight students and two fourth grade classrooms. Each fifth grade classroom in both groups has a maximum of twenty-nine students and two fifth grade classrooms. While not all classrooms will be at capacity, the sample will be large enough to have the sufficient statistical power to minimize a Type II error.

\section{Measures}

Student Achievement. Quantitatively (QUAN), this study collected student achievement data through the use of assessment procedures in which the outcome is a percentage of correctly identified answers. All students were initially assessed using curriculum-based assessment (CBA). CBA were used to help directly target specific CCSS (NGA Center, 2010) in mathematics to effectively measure student achievement in fractions. Carbaugh (2014) wrote, "teacher-designed assessments must also align with standards to ensure reliable and valid representations of student learning" (para.2). In the case of CBA, curriculum publishers have taken the guesswork out of alignment. Student proficiency data for this study will be retrieved from the measures that are a part of the curriculum used in both schools, which is GoMath: Common Core Edition (Houghton Mifflin, 2011). In the curriculum package for GoMath: Common Core Edition (Houghton Mifflin, 2011) they offer an assessment guide at each grade level. The assessment guide for each grade level includes skill inventories, benchmark assessments, diagnostic interviews, chapter tests, performance assessment, future grade preparatory assessments, individual and class forms, and correlation of items to lesson objectives. 
GoMath assessments for student achievement. For this study, the instrument used to measure student proficiency will derive from GoMath: Common Core Edition (Houghton Mifflin, 2011) chapter tests. GoMath: Common Core Edition (Houghton Mifflin, 2011) was found to have a statistically significant effect on concepts development with a .8 effect size in concepts study (Houghton Mifflin Harcourt, n.d.). In addition, GoMath: Common Core Edition (Houghton Mifflin, 2011) was found to have large effect sizes for the mathematics total results: third grade showed a fifty point gain in students' scores who used the program in comparison to a twentyfive point gain in those students' scores who did not use the program. Fourth grade showed a fifty-two point gain for those students who used the program in comparison to a thirty-seven point gain in students who did not use the program (Houghton Mifflin Harcourt, n.d.). These measures are based on publishers' research, which could potentially affect the internal validity of the study. In order to account for this threat, the researcher will measure reliability of GoMath: Common Core Edition by using Cronbach's Alpha (Cronbach, 1951). Cronbach alpha is an internal validity measure to help predict reliability of assessment items (Cronbach, 1951; Tavakol \& Dennick, 2011).

GoMath: Common Core Edition (2011) chapter tests consist of two forms, Form A and Form B. Form A contains twenty-five multiple-choice questions aligned to CCSS. Form B includes twelve multiple-choice questions, thirteen written responses, one constructed response, and two extended response questions all aligned with CCSS. Figure 3.10 offers examples of the assessment questions found in Form A at each grade level. 
Figure 3.10. GoMath: Common Core Edition (2011) Form A Question Examples Form A Item Example $3^{\text {rd }}$ Grade

Dan and David are on the track team. Dan runs $\frac{1}{4}$ mile each day. David runs $\frac{3}{4}$ mile each day. Which statement is correct?

A. Dan runs farther than David each day.

B. David runs more than 1 mile each day.

C. David runs the same distance as Dan each day.

D. David runs farther than Dan each day.

\section{Form A Item Example $4^{\text {th }}$ Grade}

Wanda rode her bike $\frac{21}{10}$ miles. Which mixed number shows how far Wanda rode her bike?

A. $\quad 1 \frac{1}{10}$ Miles

B. $\quad 1 \frac{2}{10}$ Miles

C. $2 \frac{1}{10}$ Miles

D. $2 \frac{10}{10}$ miles 


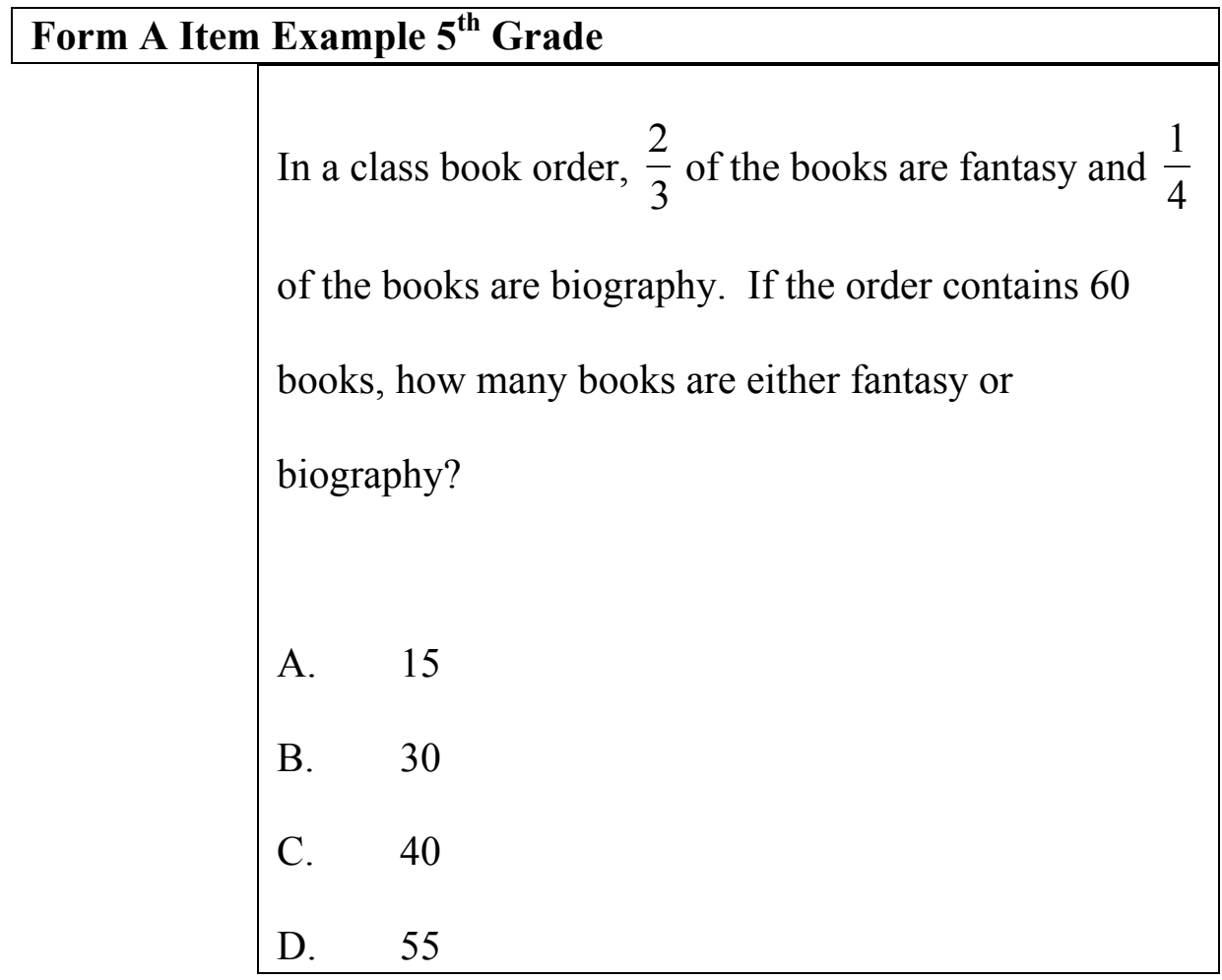

Figure 3.10. This figure gives examples of questions students will find on their GoMath: Common Core Edition pre and posttest assessment in grades 3 through 5 (Houghton Mifflin, 2011).

In the assessment guide there is an overview of the alignment with the Common Core State Standards. On this data table teachers can quickly analyze what lesson each item was taught, with which CCSS the item aligns, what the common errors are for these items, how teachers can intervene, and how teachers can extend the learning for advanced learners. Table 3.8 illustrates a comparison level of each form and alignment items. 
Table 3.8. GoMath: Common Core Edition (2011) Assessment Guide Chapter Tests

\begin{tabular}{|c|c|c|}
\hline Form $A$ & Form B & Item Alignment \\
\hline $\begin{array}{l}\text { Twenty-five } \\
\text { multiple-choice } \\
\text { questions. }\end{array}$ & $\begin{array}{l}\text { - Twelve multiple- } \\
\text { choice questions. } \\
\text { - } \begin{array}{l}\text { Thirteen written } \\
\text { responses. }\end{array} \\
\text { - One constructed } \\
\text { response. } \\
\text { Two extended } \\
\text { response questions }\end{array}$ & $\begin{array}{l}\text { - Common Core } \\
\text { - Ltandard. } \\
\text { - } \text { Lesson taught. } \\
\text { - Interventions. } \\
\text { - Extensions. }\end{array}$ \\
\hline
\end{tabular}

Note. This table shows the chapter test layout in the GoMath Assessment Guide: Common Core edition. Both forms of the chapter test are aligned with Common Core Standards (Houghton Mifflin Harcourt, n.d.), curriculum chapter lessons, interventions, and extensions for learning.

Rationale for curriculum-based assessment. Kilpatrick and colleagues (2001) suggest, "whether externally mandated or developed by the teacher, [assessment] should support the development of students' mathematical proficiency" (p. 423). Curriculum-based assessments have effectively offered insight into student understanding and mathematics achievement (Kelley, Hosp, \& Howell, 2008). Keller-Margulis, Shapiro, and Hintze (2008) found the predictors of CBA scores on statewide achievement test score as well as norm-referenced test to have a $71.06 \%$ accuracy rate on fifth grade computation and a $71.58 \%$ accuracy rate on concepts and application.

Other widely used national assessments such as the Measure of Academic Progress (Northwest Evaluation Association (NEA), 2003), Terra Nova (Data 
Recognition Corporation, 2007), and the Group Mathematics Assessment and Diagnostic Evaluation (Pearson, 2010) have been used in other large-scale studies to measure student academic performance. However, these assessments are standardized tests that measure all domains of the CCSS (NGA Center, 2010) with limited items on Numbers and Operations: Fractions. Using assessments such as these may fail to localize the needed student understanding in fractions. In a study completed by Andren (2010) found that when using both Measures of Academic Progress (NEA, 2003) and CBA in measures of reading progress, CBA was likely to provide similar if not the same results. Therefore, the use of GoMath: Common Core Edition Chapter Tests to measure student achievement should yield similar results as those found in the Andren (2010) study. Thus, helping to improve the generalizability of this study to other schools and populations.

Classroom instruction and mathematics practice. The Reformed Teaching Observation Protocol (RTOP) (Sawada, Piburn, \& Judson, 2010) will be used to observe participating teachers. The RTOP has a reliability score of a 95 (Piburn \& Sawada, 2000). Teachers will each be observed three times using the RTOP. The RTOP rates important instructional qualities inside the classroom during the instructional period. These qualities are defined in three main categories and narrowed further within those categories. These categories are identified in Table 3.9. 
Table 3.9. Reformed Teaching Observation Protocol (RTOP) Categories

$\underline{\text { RTOP Category }}$

- Lesson plan and

implementation

- Content

- Classroom Culture

\section{Sub-Category}

- Propositional

- Procedural knowledge

- Communicative indicators

- Student/teacher relationships

Note. This table identifies observation categories found on the RTOP Instrument.

The qualities found on the RTOP look at students' prior knowledge, the overall lesson design, creativity, flexibility in problem solving, and the determination of fundamental concepts of the lesson. These qualities are scored on a scale of $0-4 ; 0$ being never occurred and 4 being very descriptive. The categories of instruction indicated on the RTOP and displayed in table 3.9 have impact on student achievement (Kilpatrick et al., 2000). Figure 3.11 shows sample items from the RTOP. 
Figure 3.11. Items on the Reformed Teaching Observation Protocol (RTOP)

\section{Content - Procedural Knowledge (STUDENTS)}

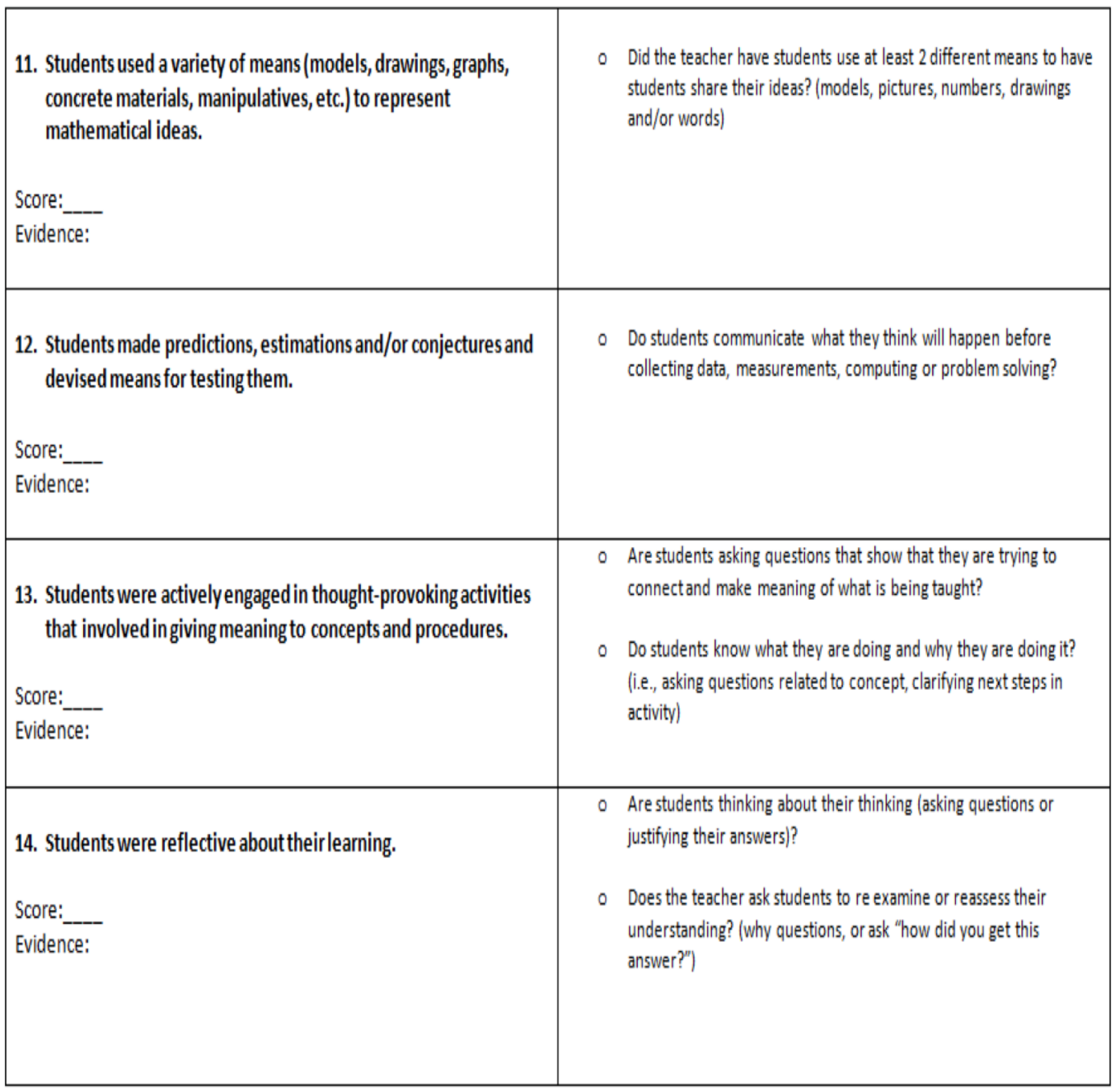

Figure 3.11. shows items found on the Reformed Teaching Observation Protocol (RTOP) (Sawada et al., 2010).

Using the RTOP helped to analyze the impacts of instruction on both the treatment and control group. Explaining the qualities of instruction and learning environment in the observed classrooms helped to inform, whether any observed, increases or significant differences in student achievement can be attributed to the implementation of interactive technology using the GoClass application. 
Rationale for using classroom observation. In a study conducted by Kennewell, Tanner, Jones, and Beauchamp (2007) the researchers observed the classroom use of interactive whiteboards. Observations using the Analyzing Teaching and Learning in Activity Settings (ATLAS) framework revealed specifically the interactivity level between student and interactive whiteboard, as well as with the teacher (Kennewell, Tanner, Jones, \& Beauchamp, 2007). Observation is a powerful tool that can uncover the specifics of an event. For this study students were observed during the treatment and control group sessions. The RTOP will allow the researcher to explain significant differences in instruction and learning environment.

Validity and Reliability. While this study will occur in a normal educational setting the researcher will review the importance of validity in the design of the study in regards to the QUAN data collected. Shadish and colleagues (2002) use the term validity "to refer to the approximate truth of an inference" (p. 34). Shadish and colleagues (2002) go on to explain the importance of valid inferences and conclusions and generality to the population.

RTOP has a reliability score of a .95 (Piburn \& Sawada, 2000) and Cronbach Alpha (Cronbach, 1951) will be use to help predict the reliability of the CBA GoMath: Common Core Edition. In addition, to ensure the normality assumption is not violated, the researcher will report the skewness and kurtosis of the data if they exist. This will help to improve the generalizability of the study to other schools or populations. In addition, the treatment implementation will be easily monitored with reports that are provided within the GoClass application. This will help to minimize threats of implementation. 


\section{Statistical Analysis}

QUAN data will be analyzed using a one-way analysis of covariance (ANCOVA). Pretests will be the covariate and the analysis will uncover the results of the impact of the tablet technology. ANCOVA has the following assumptions: independence, normality, equal variance, independence of the covariate, and homogeneity of regression slopes.

For this analysis the instructional method will be the independent variable with two levels, treatment and control. Student proficiency on fractional knowledge will be the dependent variable. Pretests will serve as the covariate. Pretest will establish the current level of students' performance. A F test will examine equal variances between groups. When examining treatment and control groups, differences will exist as part of a normal classroom. In order to isolate the effect of the treatment students' current level of fractional knowledge should be taken into consideration. The co-variate, pre-test, will help to maintain independence of variance between groups. The pretest scores will explain some variance in both the treatment and control groups. Normality will be checked for skewness and kurtosis in analysis. Homogeneity of regression of slopes will be used "to assure that the outcome and the covariate is the same in each of our treatment groups" (Field, 2009, p. 413). An SPSS model will be run to ensure this assumption was not violated.

The RTOP will be used to look at classroom differences between treatment and control groups. Minimal differences should exist between RTOP scores. The 
RTOP scores will be measured using a linear regression model. The regression equation used:

$$
\widehat{Y}=\bar{Y}-b_{y * X} \bar{X}+b_{y * X} X
$$

The researcher expects a relatively straight line between measures. This should indicate that treatment and control groups instructional environment are equal and comparable.

\section{Summary}

This chapter addressed the research design of the study. This chapter accounted for the population, sample, treatment, treatment and control groups, variables, and instruments. In addition, this chapter gave an account of the statistical analysis that is pending the completion of data collection. The goal of this chapter was to specifically explain how the research question is to be answered. 


\section{CHAPTER IV}

\section{RESULTS}

The purpose of chapter 4 is to present the findings of the RTOP scores, the fidelity of treatment, the reliability of the assessment measure, GoMath, and provide the ANCOVA results to answer the research question. The RTOP scores were measured and analyzed using a repeated measures one-way analysis of variance. This chapter provides the results of this analysis and determines if the two groups (treatment and control) were in fact comparable in regards to the teaching and learning environment. To determine that the treatment had a true effect, treatment must have been implemented with fidelity. This chapter discusses the fidelity of treatment. The reliability of the GoMath assessment was examined using Cronbach's alpha. This chapter discusses the results of this reliability measure. Lastly, the effect of treatment was measured using an ANCOVA. This chapter discusses the results of the ANCOVA and provides an answer to the research question.

In summary, this chapter shows that the teaching and learning environments in both the treatment and control group are not different. This finding will help to establish that any differences in posttest scores are due to the treatment effect and not differences in the teaching and learning environments. This chapter will establish the 
fidelity of treatments and the reliability of the testing instrument. Lastly, this chapter will answer the research question with the results of the ANCOVA.

\section{Purpose and Research Question}

The purpose of this study is to examine the impact of instruction using an interactive tablet application on student achievement in fractions. Students were provided with the opportunity to construct their own knowledge using the interactive tablet application, to support their achievement in developing fractional concepts (Means \& Olson, 2004; Manzo, 2010; \& Marzano \& Haystead, 2009).

\section{Research Question:}

How will elementary student achievement be impacted by the use of an interactive tablet application designed to facilitate student construction of responses to fraction problems?

\section{Sample}

A total of 357 students in grades three through five in a large urban midwestern school district participated in this study. The study was conducted from February 2015 until May 2015. A total of 99 students were removed from the study because of missing test scores. Missing test scores were the results of students transferring schools or not completing either the pre- or post-test. After accounting for missing data, a total of 258 scores were analyzed. Table 4.1 represents the total number of pre-post-test scores that were analyzed in this study. 
Table 4.1. The Number of Pre-Post-Test Scores for Treatment and Control

Grade Treatment Control

$\begin{array}{lcc}3^{\text {rd }} & 46 & 51 \\ 4^{\text {th }} & 40 & 40 \\ 5^{\text {th }} & 40 & 41 \\ \text { Total } & 126 & 132\end{array}$

Note. The power analysis determined that with six groups (2 classrooms at each of grades three, four and five) a minimum sample size of 162 participants would have adequate power with an effect size of .4, alpha level at .05, power at .95 . The number of scores included in data analysis is 263 providing sufficient statistical power.

A power analysis was conducted and it was determined that with six groups (2 classrooms at each of grades three, four and five) a minimum sample size of 162 participants would have adequate power with an effect size of .4, alpha level at .05, power at 95 . After accounting for missing data, 258 students fully participated in the study. This provided sufficient power to assist in detecting if a treatment effect exists.

\section{Teaching and Learning Environment}

The following sections discuss the results of the RTOP scores. First, the researcher compared both groups across all three grade levels. Second, the researcher combined fourth and fifth grade scores because the sample only consisted of one teacher at each level. The small number of teachers required fourth and fifth grade to be combined in order to run a statistical test to look at differences across groups.

Lastly, the researcher looked at raw mean scores at all levels. The researcher wanted 
to note any differences in raw mean scores to help further explain any differences found in posttest fractions scores. RTOP comparisons were done to assist in determining that the teaching and learning environment of both treatment and control groups were similar. The researcher wanted to affirm that any differences between the two groups were in fact due to the treatment effect and not a result of better or different instruction or classroom environment.

RTOP Scores. Observations were completed in both treatment and control groups as also detailed in Chapter three. Observations were completed to determine that the classroom teaching and learning environment were comparable across both groups. In third grade, in both groups, all third grade teachers teach mathematics. Therefore, all third grade teachers in both groups were observed three times over the study period. Both schools departmentalize for mathematics instruction in fourth and fifth grade. Therefore, only the teacher of mathematics was observed three times at those grade levels, over the observation period outlined in chapter three.

RTOP scores were analyzed with SPSS software using a repeated measures one-way analysis of variance. First, overall scores on the RTOP were entered into an Excel spreadsheet. The Excel spreadsheet included the variables, group (treatment/control), teacher (each teacher was coded by grade level), and observation 1, 2, and 3. The researcher used this information to enter data into SPSS.

Overall Observations. Observations were conducted at grades three through five and analyzed at this level to account for differences between treatment and control groups in the teaching and learning environment on the categories that are components of the RTOP instrument. Observation sessions initially included fractions 
instruction and by the last observation teachers were on different mathematics domains depending on grade level. A repeated measure one-way analysis of variance was used in order to compare teaching and learning environments. Mauchly's Test of Sphericity was considered to assume equal variances. Mauchly's Test of Sphericity was not significant $(p=.945>.05)$ at the whole group, grades 3-5 level. Therefore, equal variance is assumed.

Wilks' Lambda indicated that when comparing the observation to the assigned group (treatment or control) there was not a statistically significant difference $(p=.262>.05)$ when alpha is set at .05 as seen in Table 4.2.

Table 4.2. Repeated Measures for Differences Between Treatment and Control Groups

\begin{tabular}{lcccccc}
\hline Effect & & Value & F & Hypothesis df & Error df & Sig. \\
\hline $\begin{array}{l}\text { Observati } \\
\text { on*treatc } \\
\text { on }\end{array}$ & Wilks' Lambda & .682 & 1.629 & 2.0 & 7.0 & .262 \\
& & & & & & \\
\end{tabular}

Note. $\mathrm{p}<.05$. No statistically significant differences are seen in the treatment and control groups from the RTOP observation scores in grades three through five.

The implication of this finding indicates that any differences between treatment and control scores on student achievement in grades 3-5 cannot be attributed to differences in teaching or classroom environment. A repeated measure general linear model was also analyzed at each specific grade level to ensure no grade level difference exists.

Third Grade Observations. Each grade level was analyzed independently to ensure that the teaching and learning environment of the treatment and control 
groups were comparable at each level. Third grade RTOP scores were analyzed using a repeated measures one-way analysis of variance. Mauchly's Test of Sphericity was analyzed to ensure equal variances. Mauchly's Test of Sphericity was not significant $(p=.838>.05)$ when alpha was set at .05 . Therefore, equal variances were assumed in this test.

Wilks' Lambda indicated that when comparing the observation to the assigned group (treatment or control) there was not a statistically significant difference ( $p=$ $.146>.05$ ) when alpha is set at .05 , as reported in Table 4.3.

Table 4.3. Repeated Measures for Differences Between Treatment and Control Groups

\begin{tabular}{lcccccc}
\hline Effect & & Value & F & Hypothesis df & Error df & Sig. \\
\hline $\begin{array}{l}\text { Observatio } \\
\text { n*treatcon }\end{array}$ & Wilks' Lambda & .645 & .827 & 2.0 & 3.0 & .518 \\
\end{tabular}

Note. $\mathrm{p}<.05$. No statistically significant differences are seen in the treatment and control groups from the RTOP observation scores in grades three.

The implication of this finding indicates that any differences between treatment and control scores on student achievement in grade three cannot be attributed to differences in teaching or classroom environment as indicated in the categories found in the RTOP instrument.

RTOP Raw Scores. Table 4.4 shows RTOP observation raw scores. While the RTOP scores did not indicate any statistically significant differences, RTOP raw score means are still included. Looking at raw score means will determine if any meaningful differences do exist in RTOP observations. 
Table 4.4. Third Grade RTOP Raw Scores for Treatment and Control Groups

\section{Treatment}

\section{Control}

Observation 1

- Teacher A

- Teacher B

- Teacher $\mathrm{C}$
57

52

59
76

59

56

Observation 2

- Teacher A

- Teacher B

- $\quad$ Teacher C
56

53

63

26

65

49
81

50

45

Observation 3

- Teacher A

- Teacher B

- Teacher $\mathrm{C}$

\begin{tabular}{cc}
26 & 81 \\
65 & 49 \\
49 & 47 \\
\hline $53(s d=1.63)$ & $60(s d=1.33)$
\end{tabular}

\section{RTOP Mean}

Note. This table shows the combined raw RTOP scores for each area; lesson planning, content, and classroom culture.

Teachers were not matched across treatment and control groups. Rather, they were randomly assigned a label, Teacher A, Teacher B, or Teacher C. Raw RTOP scores were added and then averaged using the standard mean formula. Raw scores 
indicate that the Control group had a seven-point difference in teaching and learning environment over the treatment group.

Fourth and Fifth Grade Observations. Fourth and Fifth grade samples were too small to run statistical tests in isolation. Only one teacher in the treatment group and one teacher in the control group could be compared at fourth grade and then at fifth grade. Therefore, the researcher chose to combine fourth and fifth grade scores. Fourth and fifth grade scores were also analyzed using a repeated measures one-way analysis of variance. First, equal variance was measured using Mauchly's Test of Sphericity. Mauchly's Test of Sphericity was not found significant $(p=.679>$ $.05)$. Therefore, equal variances can be assumed.

Wilks' Lambda indicated that when comparing the observation to the assigned group (treatment or control) there was not a statistically significant difference $(p=$ $.798>.05)$ when alpha is set at .05, as reported in Table 4.5 .

Table 4.5. Repeated Measures for Differences Between Treatment and Control Groups

\begin{tabular}{llllll}
\hline Effect & Valu & F & Hypothesis & Error df & Sig.
\end{tabular}

e df

\begin{tabular}{lllllll}
\hline $\begin{array}{l}\text { Observatio } \\
\text { n*treatcon }\end{array}$ & Wilks' Lambda & .637 & .285 & 2.0 & 1.0 & .798
\end{tabular}

Note. $\mathrm{p}<.05$. No statistically significant differences are seen in the treatment and control groups from the RTOP observation scores in grades three.

The implication of this finding indicates that any differences between treatment and control scores on student achievement in grades four and five may not 
be attributed to differences in teaching or classroom environment as seen in the categories outlined in the RTOP instrument.

RTOP Raw Scores. The fourth and fifth grade RTOP scores were too small to run an independent statistical analysis. However, the researcher still finds importance in the RTOP raw scores for both fourth and fifth grade at treatment and control groups.

Fourth Grade Raw Scores. Fourth grade raw scores are shown in table 4.6. This table shows data across observation periods and the mean score of both treatment and control groups.

Table 4.6. Fourth Grade RTOP Raw Scores for Treatment and Control Groups

\section{$\underline{\text { Treatment } \quad \text { Control }}$}

Observation 1

Observation 2

Observation 3

RTOP Mean
76

75

68

$73(s d=4.36)$
57

54

62

$58(s d=4.04)$

Note. This table shows the combined raw RTOP scores for each area; lesson planning, content, and classroom culture.

While there is a difference between groups and treatment group ranks higher on RTOP observation scores, the difference is less than fifteen points. The researcher recognizes that based on any given day mathematics lesson planning, content, and even classroom culture can vary depending on many variables (i.e. classroom disruptions, approaching holidays, etc.). 
Fifth Grade Raw Scores. Fourth grade raw scores are shown in table 4.7. This table shows data across observation periods and the mean score of both treatment and control groups.

Table 4.7. Fifth Grade RTOP Raw Scores for Treatment and Control Groups

\section{Treatment Control}

Observation 1

79

22

Observation 2

87

20
Observation 3

75

$80(s d=6.11)$

12

RTOP Mean

\begin{tabular}{cc}
75 & 12 \\
\hline $80(s d=6.11)$ & $18(s d=5.29)$
\end{tabular}

Note. This table shows the combined raw RTOP scores for each area; lesson planning, content, and classroom culture.

There is a difference between groups and treatment group ranks higher on RTOP observation scores with a difference of 62 points. The researcher recognizes that based on any given day mathematics lesson planning, content, and even classroom culture can vary depending on many variables (i.e. classroom disruptions, approaching holidays, etc.).

\section{Summary of Teaching and Learning Environment}

The observations completed at both the treatment and control groups indicate that these classrooms were equal and comparable in the items measured specifically on the RTOP as discussed in Chapter three and shown in table 4.8. 
Table 4.8. Reformed Teaching Observation Protocol (RTOP) Categories

\begin{tabular}{|l|l|}
\hline \multicolumn{1}{|c|}{ RTOP Category } & Sub-Category \\
\hline$\bullet$ Lesson plan and implementation & \\
\hline$\bullet$ Content & $\bullet$ Propositional \\
& $\bullet$ Procedural knowledge \\
\hline$\bullet$ Classroom Culture & $\bullet$ Communicative indicators \\
& $\bullet$ Student/teacher relationships \\
\hline
\end{tabular}

Note. This table identifies observation categories found on the RTOP Instrument.

Repeated measures one-way analysis of variance determined that no statistical

significance exists in the RTOP scores. No difference in lesson planning and

implementation, content, or classroom culture is assumed. Therefore, the researcher

can assume statistically that any differences in student achievement scores could be the result of treatment implementation.

\section{Fidelity of Implementation}

GoClass. The fidelity of the interactive tablet application was monitored through built in systems in the application. For each instructional session during math workshop in the third, fourth, and fifth grade, GoClass provided a report that indicated how many students started and completed the session. For every instructional session a student completes, one record is generated in the logs and reports.

Student records were exported to an Excel file from the GoClass application by the company developers. In the Excel file was each student's name, user login 
information, whether they had started the instructional session and if they completed the instructional session. In addition, the developers provided a summary of the session details. This summary included the title of the instructional session, dates, number of students started, and number of students completed.

One-hundred twenty-eight students in group A participated in the study. For all students to start and complete all ten instructional sessions the total number of records produced should have equaled 1, 280 as each student would generate ten records. The number of actual records produced by all students during the study equaled 985 . Therefore, $77 \%$ of the students in group A completed the required number of instructional sessions during math workshop. Twenty-three percent of students did not complete the required number of instructional sessions for reasons such as absences and pullout interventions.

Based on the given fidelity data there could be some interference in statistical conclusion validity. Statistical conclusion validity ensures that the researcher is making a valid inference regarding the collected data (Shadish, Cook, \& Campbell, 2002). Unreliability of treatment implementation could have an affect on test results, as not all students completed the sessions with fidelity. Some students did not complete all instructional sessions due to things beyond the researcher's control. Those students may have still participated in the collection of pre and posttest scores. However, their results may not demonstrate a treatment effect because they did not complete all instructional sessions. 


\section{Reliability of the GoMath: Common Core Edition Assessment}

As noted in Chapter 3, the test publisher distributed the only available reliability data on the chosen assessment tool. Therefore, a Cronbach's Alpha was used to ensure this was in fact a reliable assessment. Cronbach's Alpha was completed for each grade level assessed. A summary of this data is seen in Table 4.9. Table 4.9. Summary of Cronbach Alpha Coefficients for Reliability

\section{Grade Level}

$3^{\text {rd }}$

$4^{\text {th }}$

$5^{\text {th }}$

\section{Cronbach Alpha}

.854

.758

Note. Demonstrates the reliability data for GoMath: Common Core Edition

Third Grade GoMath: Common Core Edition. Reliability for the third grade GoMath: Common Core Edition assessment was completed using an item analysis in SPSS and determining Cronbach's Alpha. For third grade, the Cronbach's Alpha showed a strong coefficient at a .889. Only third grade post-assessments from the control group were used to determine reliability because the test was identical at both treatment and control groups. The researcher assumed that using one group would provide a significant number of data to determine reliability. Cronbach's alpha indicates that the GoMath: Common Core Edition assessment for third grade was in fact a reliable assessment in determining student achievement in fractions.

Fourth Grade GoMath: Common Core Edition. Reliability for the fourth grade GoMath: Common Core Edition assessment was completed using an item 
analysis in SPSS and determining Cronbach's Alpha. For fourth grade, Cronbach's Alpha showed a strong coefficient at a .854 .

Fifth Grade GoMath: Common Core Edition. Reliability for the fifth grade GoMath: Common Core Edition was completed using an item analysis in SPSS and determining Cronbach's Alpha. For fifth grade, Cronbach's Alpha showed a strong coefficient at a .758 .

\section{Summary of Reliability}

In summary, Cronbach's Alpha helped to determine that GoMath: Common Core Edition was in fact a reliable assessment to use to determine student achievement in fractions. Having a reliable assessment helps to support the results of the statistical tests performed to determine significant differences amongst groups.

\section{ANCOVA Analysis}

To address the research question, means were tested using a one-way analysis of covariance (ANCOVA) at each grade level. A covariate was used to help account for a students' previous understanding in fractions. The covariate for this study was the pre-test that was given to treatment and control groups prior to fractions instruction.

ANCOVA Assumptions. ANCOVA has the following assumptions: (1) independence, (2) normality, (3) equal variance, (4) independence of the covariate, and (5) homogeneity of regression slopes.

Independence. The relationships of groups are independent of one another because the treatment will only occur in one school. The control group will be observed at an entirely different school. 
Normality. Normality was observed by completing a histogram of all scores at each grade level. Figure 4.1 illustrates that the data collected on the third grade posttest scores were normally distributed. Figure 4.2 illustrates that the data collected on fourth grade posttest scores was normally distributed. Figure 4.3 illustrates that the data collected on fifth grade posttest scores was also normally distributed.

Figure 4.1. Histogram of $3^{\text {rd }}$ Grade Posttest Scores.

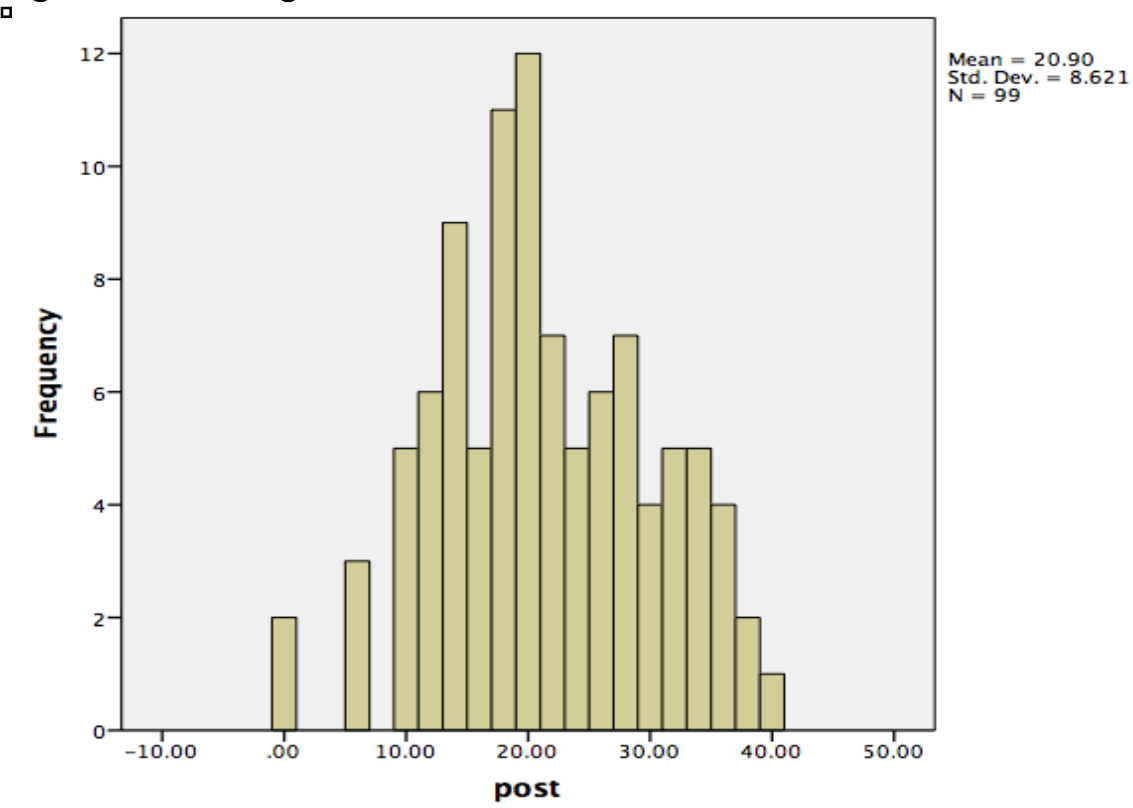

Figure 4.1. Illustrates posttest scores were normally distributed. 
Figure 4.2. Histogram of Fourth Grade Posttest Scores

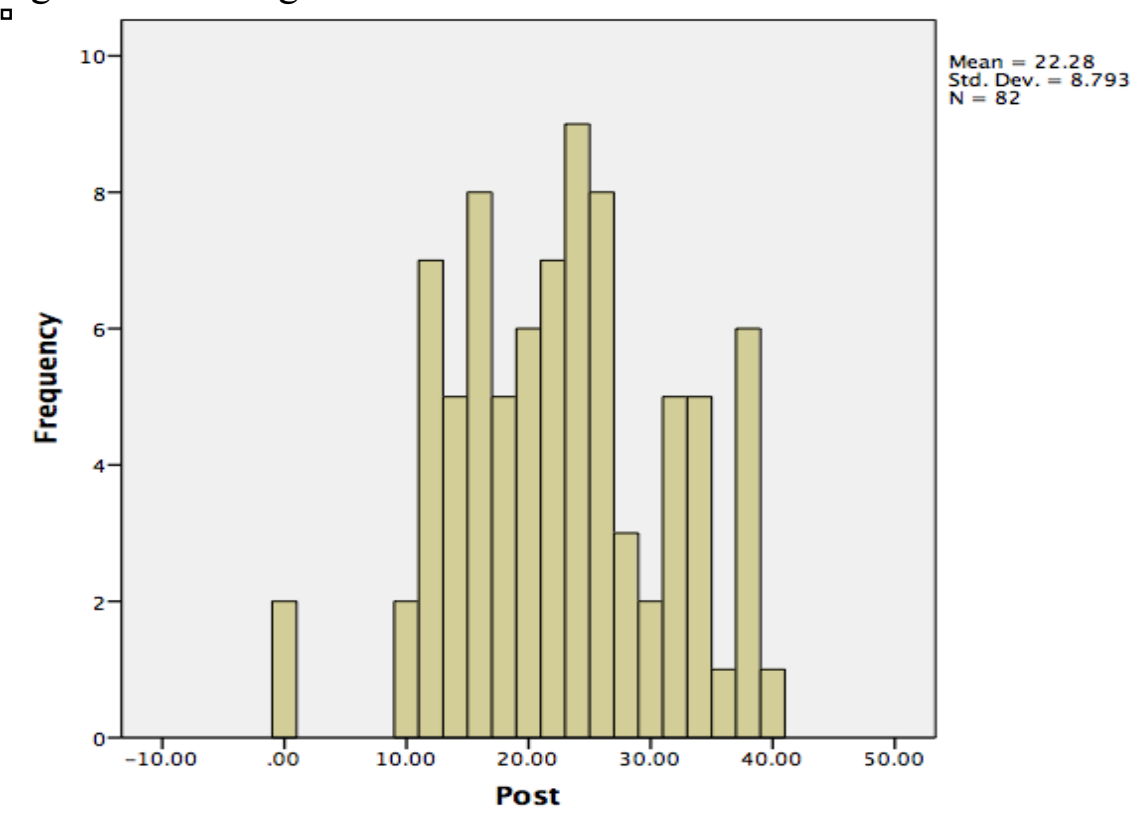

Figure 4.2. Illustrates posttest scores were normally distributed.

Figure 4.3. Histogram of Fifth Grade Posttest Scores

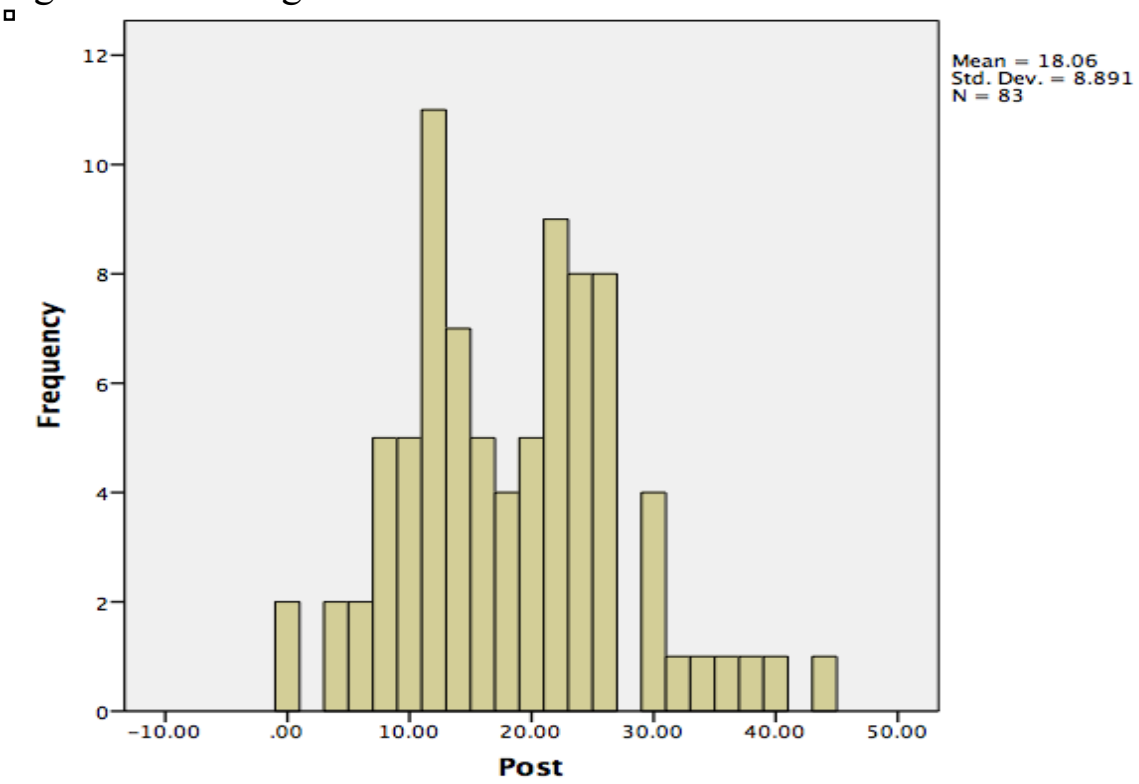

Figure 4.3. Illustrates posttest scores were normally distributed. 
Equal Variance. Equal variance is assumed because groups are mostly equal across treatment and control groups and in each grade level. Equal groups are demonstrated in Table 4.1.

Independence of the Covariate. The covariate is the pretest. The pretest was given prior to starting any treatment effect. Students were not exposed to fractions content in either group prior to taking the pretest. The covariate, pretest, is therefore independent from the experimental effect.

Homogeneity of Regression Slopes. Homogeneity of regression of slopes is addressed in the ANVOCA of each grade level and as a whole group analysis.

\section{Whole Group Grades 3-5}

Descriptive Statistics. To account for differences between treatment and control groups the researcher examined whole group differences initially to determine if there was a statistically significant treatment effect across groups. Whole group descriptive statistics are shown in Table 410. These descriptive statistics look at the mean score for both treatment and control as well as the cumulative mean scores. The sample size demonstrates there were 258 total scores that were analyzed supporting the needed number of 162 total participants for sufficient power. 
Table 4.10. Posttest Descriptive Statistics for Treatment and Control Groups

\begin{tabular}{|c|c|c|c|c|c|}
\hline Group & Grade & $\begin{array}{c}\text { Number of } \\
\text { Teachers }\end{array}$ & Mean & SD & $\mathbf{n}$ \\
\hline \multirow[t]{4}{*}{ Control } & 3 & 3 & 22.84 & 8.10 & 51 \\
\hline & 4 & 1 & 24.20 & 8.20 & 40 \\
\hline & 5 & 1 & 17.49 & 9.90 & 41 \\
\hline & Total & 5 & 21.59 & 9.08 & 132 \\
\hline \multirow[t]{5}{*}{ Treatment } & 3 & 3 & 19.65 & 21.48 & 46 \\
\hline & 4 & 1 & 21.48 & 8.00 & 40 \\
\hline & 5 & 1 & 19.55 & 6.87 & 40 \\
\hline & Total & 5 & 20.20 & 7.644 & 126 \\
\hline & Total & 10 & 20.91 & 8.42 & 258 \\
\hline
\end{tabular}

Note. $\mathrm{SD}=$ Standard Deviation

ANCOVA. Table 4.10 shows the results of the ANCOVA for treatment and control groups with pretest as a covariate. Analysis was also done on the effect of pretest scores to posttest scores. 
Table 4.11. Analysis of Covariance Against Treatment and Control

\begin{tabular}{cccccc}
\hline Source & $\underline{\mathbf{S S}}$ & $\underline{\mathbf{d f}}$ & $\underline{\text { MS }}$ & $\underline{\text { F Value }}$ & $\underline{\text { Sig. }}$ \\
Pre $_{\mathrm{COV}}$ & 5951.33 & 1 & 5951.33 & 135.57 & .000 \\
Group $_{\mathrm{TC}}$ & 96.42 & 1 & 96.42 & 2.20 & .140 \\
Grade & 518.12 & 2 & 259.06 & 5.9 & .003 \\
Group*Grade & 430.60 & 2 & 215.30 & 4.90 & .008 \\
Error & 11018.36 & 251 & 43.99 & & \\
Total & 18232.95 & 257 & & & \\
\hline
\end{tabular}

Note. $\mathrm{P}<.05$. Pre $\mathrm{COV}=$ Pretest as the Covariate. Group $\mathrm{TC}_{\mathrm{TC}}=$ Treatment and Control Group.

The covariate, pretest, was found to have a statistically significant effect on posttest scores, $\mathrm{F}(1,257)=135.57, p=.000<.05$. This indicates that the score a student received on his or her pretest would be a good indicator of how he or she would perform on his or her posttest. Therefore, the assumption of homogeneity of regression of slopes is met. Parameter estimates indicate that as pretest scores increase one unit, post test scores should increase by almost three-quarters of a unit $(b=.73)$

The variable, group, was not found to have a statistically significant effect on posttest scores, $\mathrm{F}(1,257)=2.20, p=.140>.05$. This indicates the treatment of an interactive tablet application had no significant impact on fractions learning. Effect size was also calculated using Partial Eta Squared. For treatment group partial eta squared is found to be less than one. The variable Group had less than a $1 \%$ chance of having an effect on post-test scores. 
A significant difference, $\mathrm{F}(2,257)=5.9, p=.03<.05$, in grade level is shown in Table 4.11. In addition, a significant effect, $\mathrm{F}(2,257)=4.90, p=.008<.05$, was found in the relationship between grade level and group. This indicates that somewhere with in these levels the treatment may have had a statistically significant effect on posttest scores. This was analyzed further at each grade level.

\section{Third Grade}

ANCOVA. Table 4.12 shows the results of the ANCOVA for treatment and control groups with pretest as a covariate. Analysis was also done on the effect of pretest scores to posttest scores.

Table 4.12. Third Grade Analysis of Covariance of Posttest Scores

\begin{tabular}{cccccc}
\hline Source & SS & df & MS & F Value & Sig. \\
Pre $_{\text {Cov }}$ & 2045.35 & 1 & 2045.35 & 46.90 & .000 \\
Group $_{\mathrm{TC}}$ & 4.44 & 1 & 4.44 & .102 & .750 \\
Error & 4099.83 & 94 & 43.62 & & \\
Total & 50523.00 & 97 & & & \\
\hline
\end{tabular}

Note. Note. $\mathrm{P}<.05$. Pre $\mathrm{COV}_{\mathrm{V}}=$ Pretest as the Covariate. Group $\mathrm{TC}_{\mathrm{TC}}=$ Treatment and Control Group.

The covariate, pretest, was found to have a statistically significant effect on posttest scores, $\mathrm{F}(1,97)=46.90, p=.000<.05$. This indicates that the score a student received on his/her pretest would be a good indicator of how s/he would perform on his or her posttest. This is consistent with the findings from the overall group scores. Pretest continues to have a positive relationship with posttest scores at this level. 
Parameter estimates indicate that as pretest scores increase one unit, post test scores should increase by almost three-quarters of a unit $(b=.925)$ Therefore, the assumption of homogeneity of regression of slopes is met.

The variable, treatment and control, was not found to have a statistically significant effect on posttest scores, $\mathrm{F}(1,97)=.102, p=.750>.05$. This indicates the treatment of interactive tablet application had no significant impact on fractions learning. The result of this required no post hoc tests. Effect size was also calculated using Partial Eta Squared. For treatment group partial eta squared is found to be less than one. Group had less than a $1 \%$ chance of having an effect on post-test scores. Parameter estimates also show that there is no differences in groups. This finding is inconsistent with overall group scores. Overall group scores indicated a significant difference at grade level somewhere in the data set. The ANCOVA findings in grade three eliminate this grade as a place for possible statistically significant differences in treatment and control groups.

\section{Fourth Grade}

ANCOVA. Table 4.13 shows the results of the ANCOVA for treatment and control groups with pretest as a covariate. Analysis was also done on the effect of pretest scores to posttest scores. 
Table 4.13. Fourth Grade Analysis of Covariance Posttest Scores

\begin{tabular}{cccccc}
\hline Source & SS & df & MS & F Value & Sig. \\
\hline Pre $_{\text {Cov }}$ & 1636.26 & 1 & 1636.26 & 36.41 & .000 \\
Group $_{\mathrm{TC}}$ & 16.30 & 1 & 16.30 & .363 & .549 \\
Error & 3460.12 & 77 & 44.94 & & \\
Total & 46969.00 & 80 & & &
\end{tabular}

Note. Note. $\mathrm{P}<.05$. Pre $\mathrm{COV}_{\mathrm{V}}=$ Pretest as the Covariate. Group $\mathrm{TC}_{\mathrm{TC}}=$ Treatment and Control Group.

The covariate, pretest, was found to have a statistically significant effect on posttest scores, $\mathrm{F}(1,80)=36.41, p=.00<.05$. This indicates that the score a student received on his/her pretest would be a good indicator of how s/he would perform on his or her posttest. Parameter estimates show positive relationship between pretest and posttest scores $(b=.71)$. This indicates that for every one unit increase on pretest scores, students should also have an almost three-quarter unit increase on their posttest scores. This is consistent with the findings from the overall group scores. Pretest continues to have a positive relationship with posttest scores at this level. Therefore, the assumption of homogeneity of regression of slopes is met.

The variable, treatment and control, was not found to have a statistically significant effect on posttest scores, $\mathrm{F}(1,80)=.36, p=.55>.05$. This indicates the treatment of an interactive tablet application had no significant impact on fractions learning. The result therefore required no post hoc tests. Effect size was also 
calculated using Partial Eta Squared. For treatment group partial eta squared is found to be less than one. The variable Group had less than a $1 \%$ chance of having an effect on post-test scores. Parameter estimates also show that there is no differences in groups. This finding is inconsistent with overall group scores. Overall group scores indicated a significant difference at grade level somewhere in the data set. The ANCOVA findings in grade four eliminate this grade as a place for possible statistically significant differences in treatment and control groups. 


\section{Fifth Grade}

ANCOVA. Table 4.14 shows the results of the ANCOVA for treatment and control groups with pretest as a covariate. Analysis was also done on the effect of pretest scores to posttest scores.

Table 4.14. Fifth Grade Analysis of Covariance Posttest Scores

\begin{tabular}{cccccc}
\hline Source & SS & df & MS & F Value & Sig. \\
Pre $_{\text {Cov }}$ & 2391.20 & 1 & 2391.20 & 55.89 & .000 \\
Group $_{\mathrm{TC}}$ & 422.35 & 1 & 422.35 & 9.87 & .002 \\
Error & 3336.94 & 78 & 42.78 & & \\
Total & 33555.00 & 80 & & & \\
& & & & & \\
\hline
\end{tabular}

Note. $\mathrm{P}<.05$. Pre $\mathrm{COV}=$ Pretest as the Covariate. $\mathrm{Group}_{\mathrm{TC}}=$ Treatment and Control Group.

The covariate, pretest, was found to have a statistically significant effect on posttest scores, $\mathrm{F}(1,80)=55.89, p=.000<.05$. This indicates that the score a student received on his/her pretest would be a good indicator of how s/he would perform on his or her posttest. Parameter estimates indicate a positive relationship $(b=.66)$ between pretest and posttest scores. An increase of more than half a unit should occur with every one unit increase of pretest scores. These findings continue to be in alignment with overall scores of both the treatment and control groups. Therefore, the assumption of homogeneity of regression of slopes is met. 
The variable, treatment and control, was found to have a statistically significant effect on posttest scores, $\mathrm{F}(1,80)=9.87, \mathrm{p}=.002>.05$. The treatment, interactive tablet application, had a significant impact on fractions learning. This indicates that the statistically significant effects shown in Table 4.11 are isolated to fifth grade. The result of this required no post hoc tests. Effect size was also calculated using Partial Eta Squared. For treatment group partial eta squared is found at .112 . The variable group at the fifth grade has about an $11 \%$ chance of having an effect on post-test scores. Parameter estimates were consistent with the overall findings for the treatment and control groups. A negative relationship $(b=-4.7)$ between treatment and posttest scores was indicated in analysis. The treatment had a $4.7 \%$ impact on posttest scores. The treatment group scored 4.7 units higher on the posttest than the control group.

\section{Summary}

This chapter presented the findings of data analysis at several levels. The data was presented that detailed that power has been reached with a sufficient number of participants in the study $(n=258)$. This chapter established through the analysis of RTOP scores that learning environments were not significantly different $(p=.262>$ $.05)$ across treatment and control groups. This indicated that any statistical differences may not be attributed to the teaching or learning environment based on the categories of RTOP. The fidelity of treatment implementation was established by showing that $77 \%$ of the students who participated in the treatment group, started and completed all ten instructional sessions on GoClass with a tablet device. This chapter 
established that GoMath: Common Core Edition assessment was also found to be a reliable instrument to measure student achievement in fractions. Cronbach alpha at each grade level demonstrated a coefficient number greater than .7 which is considered strong. Lastly, this chapter answered the research question:

How will elementary student achievement be impacted by the use of an interactive tablet application designed to facilitate student construction of responses to fraction problems?

In third and fourth grade, the instruction with an interactive tablet application was shown not to have a statistically significant effect on elementary student achievement in fractions. In fifth grade, the instruction with an interactive tablet application was shown to have a statistically significant relationship on elementary student achievement in fractions. 


\section{CHAPTER V \\ SUMMARY OF THE STUDY}

\section{Restatement of the Problem}

Studies have shown that students struggle with learning fractional concepts (Battista, 2012; Brown \& Quinn, 2007; Ebby, 2015; Kamii \& Clark, 1995; Mack, 1995; McNamara \& Shaughnessy, 2009). First, students may overgeneralize whole number strategies to fractions (Mack, 1995; Petit et al., 2010). Second, district pressures and time constraints lead teachers to teach students expiring rules in fractional concepts (Asku, 1997; Karp, Bush, \& Dougherty, 2014; Mack, 1995; McNamara, \& Shaughnessy, 2010). Students require effective instruction to develop conceptual understanding in fractions to help in later algebraic understanding (Barnett-Clarke, et al., 2010). Students should have access to many tools and strategies to help them increase their understanding in fractional concepts.

Technology has been shown as an effective tool to help increase student achievement in mathematics and other content areas (Herrera et al., 2007; Holmes, 2009; ISTE, 2007; Kent, 2006; Manzo, 2009; Marzano, \& Haystead, 2010; Means \& Olson, 1994; Morphew, 2012). NCTM (2008) has also suggested that technology may be used as an avenue to expand student thinking and reasoning in mathematics concepts. Allowing students to explore fractional concepts using technology as a tool could increase student achievement in this area. 


\section{Restatement of the Purpose \& Research Question}

The purpose of this study was to examine the impact of delivering instruction using an interactive tablet application on student achievement in fractions. Students were provided the opportunity to construct their own knowledge using the features of the interactive tablet application, to support their achievement in fractional concepts (Manzo, 2010; \& Marzano \& Haystead, 2009; Means \& Olson, 2004).

\section{Research Question}

How will elementary student achievement be impacted by the use of an interactive tablet application designed to facilitate student construction of responses to fraction problems?

\section{Review of Methodology and Approach to Analysis}

This study was a quasi-experimental design with an untreated control group with dependent pretest and posttest samples. The sample of the population for this study is two groups within two elementary schools in the southwest portion of a large urban mid-western school district. A total of 357 students in grades three through five in a large urban mid-western school district participated in this study. A total of 99 students were removed because of missing test scores. Resulting in 258 student test scores being analyzed.

The researcher examined classroom teaching and learning environments by conducting three classroom observations of each mathematics teacher using the RTOP instrument. Teacher scores were then analyzed using a repeated measures one-way analysis of variance. This helped control for teacher differences to ensure 
that any statistically significant differences in groups were due to the treatment and not a result of teaching or learning environments.

Analyses on student posttest scores on GoMath: Common Core Edition were completed using a one-way analysis of covariance. In this analysis, instructional method serves as the independent variable. Student pretest will serve as the covariate. Lastly, student proficiency on fractional knowledge will be the dependent variable. Posttest instrumentation reliability was measured and established using Cronbach's alpha.

\section{Summary of Findings}

In this study, power (an effect size of .4, alpha level at .05, power at .95) was achieved with a sufficient number of participants in the study $(n=258)$. Learning environments were not statistically significant $(p=.262>.05)$ across treatment and control groups. This established that there were no significant differences in learning environment. Reliability of the posttest instrument was established with an item analysis using Cronbach's alpha. Each grade level demonstrated a coefficient number greater than a .7. These results show that GoMath: Common Core Edition was reliable. An analysis of covariance of posttest scores demonstrated that no statistically significant differences between treatment and control groups existed at grades three and four in treatment and control groups. An analysis of covariance demonstrated a statistically significant difference in treatment and control groups at grade five. 


\section{Discussion and Implications for Mathematics Education}

\section{Conceptual Framework}

The framework for this study was organized into five areas:

- constructivist learning theory

- technology pedagogy content knowledge (TPACK)

- interactive technology

- instructional strategies with a tablet device

- $\quad$ student proficiency in the domain of Numbers and Operations: Fractions (NGA Center, 2010).

First, ideas of constructivist learning theory were used when developing student activities in fractions learning. Students in both treatment and control groups had opportunities to construct their own learning in regards to fractional understanding during math workshop. Second, this study examined how the TPACK framework could be applied to constructivist learning to help increase student proficiency in fractions. Lastly, an interactive tablet application was used to carry out the constructivist learning activities developed when applying both constructivist learning theory and the TPACK framework.

Constructivist Learning Theory and Interactive Technology. Students had opportunities in math workshop to work on constructing viable answers to mathematics challenges (Means \& Olson, 1994). In the treatment group, students could work with virtual manipulatives and virtual sketchpads to arrive at mathematical conclusions and develop deeper meaning to their previous ideas about 
fractions (Jonassen, 2000; Miller, 2012; Morphew, 2012; Piaget, 1954). In the control group, students worked with hands on manipulatives and math notebooks to help arrive at conclusions and develop deeper meaning to their previous ideas about fractions (Piaget, 1954; Vygotsky, 1978).

TPACK and Interactive Technology. In this study, students in the treatment group engaged in the interactive tablet application during math workshop. During this time teachers were working with students in small groups to help aide in student mathematical understanding. Students who were engaged in the interactive application received no immediate feedback from the teacher or application. Math workshop was conducted the same way in both treatment and control groups. Math workshop is part of common practice in the treatment and control groups. During math workshop, students who are not working directly with the teacher often participate in independent or small group activities for mathematics practice. Often these independent activities or small group activities include math games and projects. The meaningful difference in treatment and control, was that students in the treatment group used the interactive tablet application as one of their stations during math workshop. In the treatment group, fractions content was delivered on the interactive tablet application in a way that allowed students to construct their responses using technology. Experiences on the interactive tablet application should have helped students reformulate their knowledge set about fractions because the application was being used as a tool to extend their learning (Morphew, 2012; Piaget, 1954; Vygotsky, 1978). 


\section{Discussion}

Grades 3-5. Teacher observations in grades three through five found that there were no statistically significant differences in teaching and learning environment as measured by the RTOP instrument. The researcher found that in both treatment and control group's teachers were comparable in the teaching and learning environment. Lessons were structured similarly. Students interacted in similar ways in small groups, in partner pair, and independently. Students had opportunities to engage in discussion with their peers, as well as in whole group discussion with the teacher.

This study found that when examining grades three through five collectively that even when making shifts in content delivery through an interactive technology application, there was no statistically significant impact on student achievement in Numbers and Operations: Fractions $(p=.140>.05)$. Overall, students at grades three through five may have been engaged in fractions instruction in similar ways. The only exception to this was at fifth grade level. ANCOVA results demonstrated statistically significant results for the treatment group.

Table 5.1 shows that posttest means in treatment and control groups were similar. This illustrates that if students are constructing knowledge, no matter the method, posttest scores may be similar. 
Table 5.1. Treatment and Control Group Posttest Mean Comparison

\section{Treatment Mean (SD) $\quad$ Control Mean (SD)}

$\begin{array}{lcc}\text { Third Grade } & 19.65(7.9) & 22.84(8.09) \\ \text { Fourth Grade } & 21.48(8.00) & 24.20(8.1) \\ \text { Fifth Grade } & 19.55(6.86) & 17.49(9.8) \\ \text { Overall } & 20.20(7.64) & 21.59(9.08)\end{array}$

Note. This table illustrates that at most levels the control group had a higher posttest mean score than the treatment group.

Third Grade. Teacher observations in grade three found that there were no statistically significant differences in teaching and learning environment as measured by the RTOP instrument. The researcher found that in both treatment and control groups teachers were comparable in the areas of lesson plan and implementation, content, and classroom culture as indicated on the RTOP subscales.

An analysis of posttest scores for third grade using a one-way analysis of covariance demonstrated there were no statistically significant differences in treatment or control groups $(p=.750>.05)$. This means that the interactive tablet application had no significant effect on student achievement in fractions. Students at this grade level may have been engaged in fractions instruction in similar ways for both treatment and control groups. Students at both schools were given opportunities to construct their knowledge about fractions. The treatment effect, interactive technology, did not statistically give treatment students an advantage over control students. What students may have gained during treatment sessions, students in the 
control group may have also gained during their mathematics instruction. Therefore, if students are constructing their knowledge, whether they use other methods (e.g. paper, pencils, markers, manipulatives, mathematics games) or use technology, the impacts on student achievement may be equal.

Fourth Grade. When combined with fifth grade, teacher observations in grade four and five revealed that there were no statistically significant differences in teaching and learning environment as measured by the RTOP instrument.

An analysis of posttest scores for fourth grade using a one-way analysis of covariance demonstrated there were no statistically significant differences in treatment or control groups $(p=.549>.05)$. This means that the interactive tablet application had no significant effect on student achievement in fractions. Students at the fourth grade may have been similarly engaged in the construction of knowledge about fractions. Students in the control group had similar opportunities to construct knowledge about fractions as the treatment group. The treatment, interactive technology, assisted students in the treatment group in learning new ideas about fractions. However, at the fourth grade level, it appears the treatment served as a replacement to other instructional methods (e.g. paper, pencils, markers, manipulatives, mathematics games) rather than an extension or betterment of student understanding. Therefore, if students are constructing their knowledge, whether they use other methods (e.g. paper, pencils, markers, manipulatives, mathematics games) or use technology, the impacts on student achievement may not be significantly different. 
Fifth Grade. When combined with fourth grade, teacher instruction as measured with the RTOP in grade four and five revealed that there were no statistically significant differences in teaching and learning environment.

An analysis of posttest scores for fifth grade using a one-way analysis of covariance demonstrated there was a statistically significant difference in treatment and control groups $(p=.002>.05)$. This means that the interactive tablet application had a significant effect on student achievement in fractions at the fifth grade. The treatment, interactive technology, positively impacted student proficiency in Numbers and Operations: Fractions. Students using the interactive tablet application at fifth grade in the treatment group may have constructed knowledge about fractions at a higher level than students in the control group.

Students at the treatment school may have been more engaged in fractions instruction than at the control school. Students in fifth grade may have had more experiences with tablet technology. Previous experiences with technology may have created more enjoyment for the Fifth graders working on the interactive tablet application than with other instructional methods. Students in fifth grade at the treatment school have also worked on this interactive tablet application previously. Therefore, the novelty of the application may not have interfered with the content delivery. Fifth grade students who had previous experiences with the interactive tablet application may have been able to better focus on the actual content rather than being distracted by the functionality of the application.

Implications. This study offers several implications for teaching practice with technology. First, student understanding must be taken into account when 
designing effective lessons. Second, when students are given opportunities to construct knowledge with technology or with other methods they will perform similarly on posttest assessments.

Students' Current Level of Understanding. When planning for instruction, teachers must account for the students' current level of mathematical understanding. The pretest was a statistically significant indicator of how well students were going to perform on the posttest. This speaks to the importance of ensuring that all students are receiving instruction based on their current level of understanding (Piaget, 1954; Vygotsky, 1978). Students will construct their knowledge based on this level and the experiences they are offered in the learning environment. Therefore, student activities should be developed to specifically target students' level of understanding. Some students may be at higher levels of understanding than others. The interactive tablet application could be an excellent avenue to target student understanding levels specifically. Teachers would have the opportunity to create lessons based on students' current level of understanding. By directly targeting students to help fix misunderstandings through specific activities, this may increase student proficiency levels.

Teachers' Current Level of TPACK Understanding. When students are left to work independently on technology, no matter how the technology task is structured, they may not see significant gains. Students must think in meaningful ways when using technology and they must work collaboratively with their teacher to develop new learning (Jonassen, 1996). Technology should be a tool to assist in 
learning, but ultimately it may be the teacher's pedagogical practice and content knowledge used in conjunction with the technology that has the greater effect.

Technology has the ability to support effective teaching strategies and practices by providing a resource to facilitate explicit instruction, whole class discussion, small group work, and immediate feedback to students (Marzano \& Haystead, 2009; Marzano et al., 2000; University of Michigan, 2015). This was seen in Marzano and Haystead's (2009) study on instruction using the interactive whiteboard. Their study was an example of technology becoming part of the full teaching and learning process with the teacher as a catalyst. This current study supports that the teacher is still an important facilitator of learning and that technology in isolation might not be as effective.

\section{Limitations and Recommendations}

This study had three primary limitations. First, the study only analyzed the one feature of the interactive tablet application, independent instructional sessions. Second, this study did not take into account teachers' current level of TPACK. The teachers' current level of TPACK Knowledge (Niess et al., 2009) could have important implications on their ability to integrate technology into their mathematics instruction and still engage students and provide feedback. These knowledge levels are shown in Figure 5.1. Third, this study only examined student understanding in Numbers and Operations: Fractions. This study may have produced different results if a different mathematics content area had been examined. For example, student groups may have had statistically significant results if the content area of geometry had been examined rather than fractions. Fractions are a difficult concept but 
important for students to understand. Tackling an area that is more visual in nature like geometry or more of a review such as continued work with whole numbers may have produced different results. Taking these factors into account in the study could have produced different outcomes.

\section{Technology Limitations}

In this study, students worked with the interactive tablet application independently during math workshop. During math workshop teachers are focused on providing small group instruction. Students who were working independently on the interactive application received no immediate feedback or support from the teacher or the application.

While many effective teaching strategies and high-leverage practices were used within math workshop (Marzano, et al., 2001; University of Michigan, 2015), this study did not examine feedback built into the lessons with the technology application or the importance and role of teacher feedback to the students on what they completed with the application. This study did not take into account the whole group option of the interactive technology application. In the whole group option of the GoClass technology application, teachers could decide the content they would prefer to show on the screen to the whole group, as well as the content students would see on their devices. For example, when discussing part-whole relationships, teachers could choose to show a diagram on the whole class screen. The students would see a question they would have to answer either in scribble or in written form on their

tablet devices. Student answers generated on their tablets are delivered directly to the teacher device. From there, the teacher can decide to show student responses 
anonymously to generate student discussion. In addition, this feature allows teachers to go directly to students that have misunderstandings about the concepts. Using the whole group functionality in this study could have helped students in the treatment group see more significant gains at all grade levels as the feedback they would have received would have been more immediate. Without technology, feedback can be delayed. Teachers have to take time to walk through the class to examine student work on a case-by-case basis. When using technology, teachers are able to see student responses from their own device. Limiting walk around time, teachers can directly target students to eliminate misconceptions. This model aligns with Marzano and Haystead's (2009) study completed on interactive whiteboard technology that found positive gains on student achievement. If the current study had addressed the whole group functionality, additional effective teaching strategies and high-leverage practices may have been more applicable (Marzano et al., 2000; University of Michigan, 2015).

In this study, students worked independently and like the control group, received delayed feedback. Teachers in the treatment group were able to use student work from the interactive technology application to fix student misconceptions. However, this happened in the same manner as the control group. Technology was not used to deliver rapid feedback to students.

Research done on tablet devices is limited. This study will continue to add to that body of research and provide some insight on developing specific strategies when using tablet devices. These strategies may include the importance of teacher feedback as students are working. If teachers had given students feedback as they 
were constructing answers students may have had opportunities to correct

misconceptions. Thus, further developing their conceptual understanding of fractions.

\section{Teacher Limitations}

In the TPACK framework, technology is integrated into pedagogy and content knowledge (Koehler \& Mishra, 2008). This integration requires teachers to have knowledge about how this occurs in the instructional process. These levels range from recognizing to advancing (Niess et al., 2009). Figure 5.1. illustrates how teachers' thinking and understanding are connected in the TPACK framework.

First, teachers must recognize the importance of technology integration. Technology is a tool that can promote constructivist learning and higher order thinking skills (Morphew, 2012; Reinhart et al., 2011). Second, teachers must accept technology as a tool to expand student thinking and creativity (Jonassen, 2000; Morphew, 2012; Niess et al., 2009). These TPACK knowledge levels continue to the highest level of advancing.

The current level of teacher understanding of TPACK could have potential to impact how well the interactive tablet application was integrated into mathematics instruction. 
Figure 5.1. Teachers' TPACK Knowledge Levels

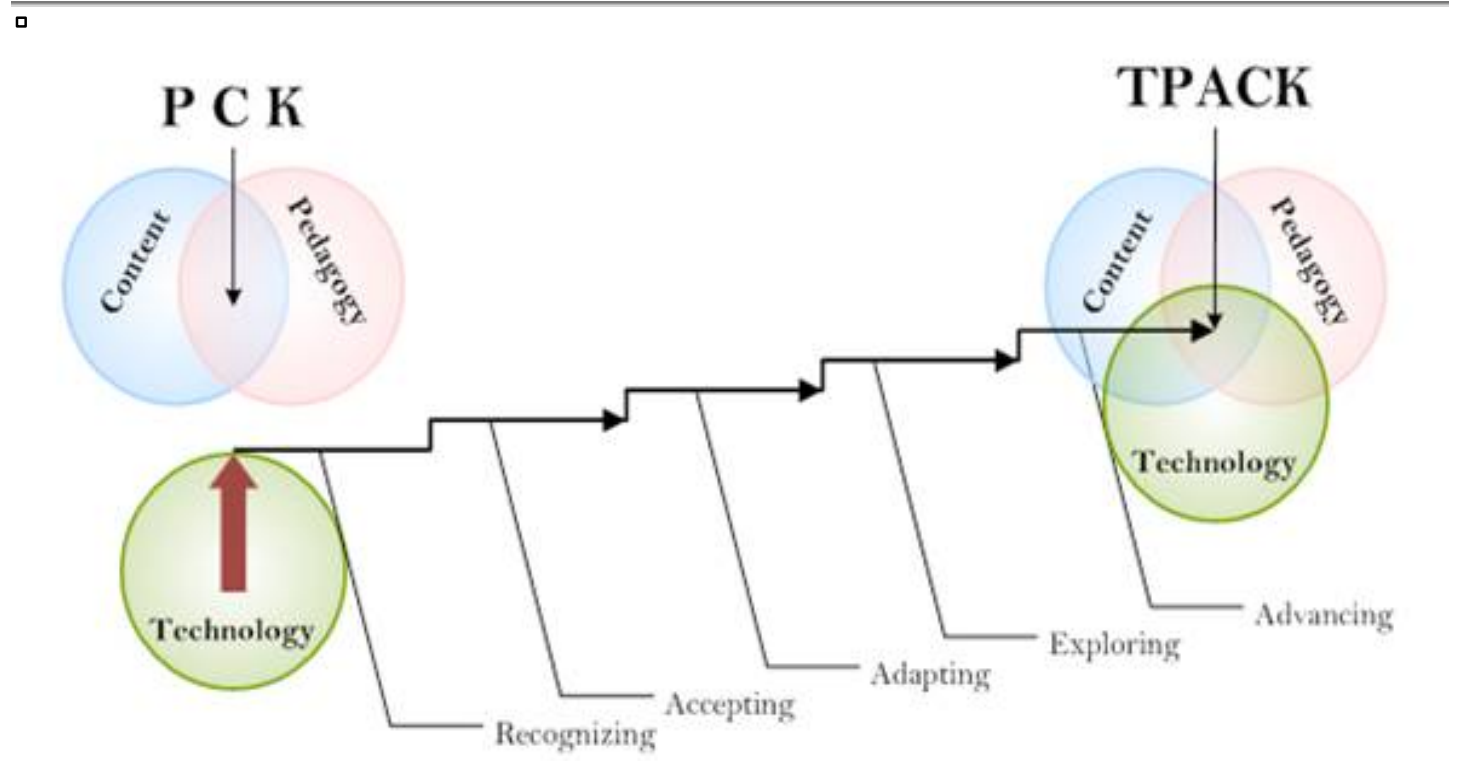

Figure 5.1. This figure illustrates a visual description of teacher knowledge levels and the path of integrating technology into their content and pedagogical practice (Niess et al., 2009).

In general, this study revealed that most students interacted with the application on an independent level during math workshop with no immediate feedback from the teacher, other students, or from the application. Teachers in this study recognize the importance of technology integration in their content and pedagogy. Each was interested in integrating this interactive technology application into their math workshop because they understood the importance of allowing students access to digital tools. When teachers are at the recognizing level of TPACK Knowledge "...teachers are able to use the technology and recognize the alignment of the technology with content yet do not integrate the technology in teaching and learning of mathematics" (Niess et al., 2009, p. 9). This study could be used as a catalyst to help move teachers to a higher TPACK knowledge level. When teachers 
understand their role in technology use and they understand the TPACK knowledge levels, they may choose to seek professional development to increase their skills when integrating technology into the teaching and learning environment. Thus, helping them move along the TPACK knowledge level trajectory.

In the future, valuable information could be gained by assessing teachers' knowledge of TPACK. This information could help guide professional development opportunities and the role that the technology facilitator would play in the implementation of technology in instruction (Reinhart, 2011). Assisting teachers in technology integration could help to increase the possibility that technology could enhance pedagogical practice, therefore, increasing student achievement. Technology should not be a tool outside of pedagogy or content, but one that is integrated seamlessly into these areas as the TPACK framework suggests (Mishra \& Koehler, 2006).

\section{Content Limitations.}

This study only examined student achievement differences in Number and Operations: Fractions. Student achievement in other areas may have produced different results. For example, if students used this interactive technology application in conjunction with applications such as Geometers Sketchpad in the area of geometry, different results may have been produced.

\section{Future Research}

Successful integration of technology comes back to the teachers' pedagogical practice and content knowledge. Suggestions for future research include replicating a similar study using a mixed-methods design. This mixed-methods design would look 
more extensively at the variables involved in the study. For example, student engagement would be measured, students' beliefs about mathematics and technology, as well as more extensive observations of treatment and control teachers using the RTOP instrument and field notes.

Student interactions could be considered using a more qualitative approach. By observing student engagement with the interactive technology application, research may be able to determine if the lack of statistically significant scores was due to lack of student engagement. Another important factor, when thinking about student roles in this study that was not considered was their own personal feelings about mathematics and technology. Students who do not like technology or who do not like mathematics may feel less inclined to engage in mathematics instruction even in a workshop model using technology.

A second recommendation for future research includes a more comprehensive look at all functions that exist in the GoClass application. The current study was based on individual student use of tablet technology using an interactive application during math workshop. Benefits may exist when coupling the independent instructional sessions and whole group teaching within the interactive tablet application during regular classroom mathematics instruction. Whole-group teaching with immediate student feedback could be measured, along with the correction of student misconceptions during whole group instruction. Field notes during whole group instruction and math workshop could be examined to draw more concise conclusions about the teaching and learning environments. 
Benefits may exist in using pre-service teachers in a field placement to examine the effects of seamless technology integration from that aspect. Preservice teachers have had limited time developing their teaching craft and experienced teachers have had many years to develop their teaching craft and may have a more difficult time adjusting their pedagogical practices to newer ideas. Pairing pre-service with inservice teachers might be an avenue to have them support each other on this integration. A mixed-methods design would allow the researcher to examine more closely the specific ways in which beginning and experienced teachers make these shifts by examining teacher beliefs and knowledge about technology.

In addition, benefits may exist at examining the teachers' current knowledge level of TPACK. A correlation may exist between the teachers' current knowledge level, level of technology integration, and student achievement scores. Examining teachers' current level of TPACK would also be beneficial, as coupling both independent instructional sessions and whole group teaching with the application requires a shift in pedagogical practices. By examining teachers' current knowledge level of TPACK, appropriate training and in-service could be targeted and delivered specifically to meet teachers' needs to assist in integrating technology into instruction. Professional development offered on effective technology integration could benefit the teachers involved by providing them resources to help shift their pedagogical practice and increase their TPACK knowledge level.

Evaluating teachers' own feelings about their pedagogical practices and effective integration of technology would give additional insight and may prove to be 
more effective in increasing student achievement, because gaps in teacher understanding could be more effectively addressed.

\section{Conclusion}

This study was a quasi-experimental design with an untreated control group with dependent pretest and posttest samples. Data were analyzed with a one-way analysis of covariance. Results indicated there were no statistically significant differences between treatment or control at grades three or four. In grade five, results indicated there was a statistically significant difference in groups on posttest scores GoMath: Common Core Edition.

The study results imply successful integration of technology is dependent on the teachers' pedagogical practice and more importantly teachers' knowledge level of TPACK and the level of student feedback received during instruction. The TPACK framework integrates technology into pedagogy and content (Mishra \& Koehler, 2006). This integration demonstrates to teachers that technology should not be something outside of these two areas, but rather a tool to help extend content and practice. Assisting teachers in their own development of technology integration through pedagogical shifts could help to increase student achievement.

This study adds to the current field of research by acknowledging important components in technology integration in mathematics. Additional research still needs to be completed to help identify the role of the teacher in this type of learning. Additional work should also be completed when looking at specific ways that students interact with the tablet technology during independent instruction. Teaching 
with technology is a rapidly-changing field, efforts to develop best research-based practices with technology should continue to help narrow the achievement gap of students and improve the TPACK level of the teacher. 


\section{REFERENCES}

Aksu, M. (1997). Student Performance in Dealing with Fractions. Journal of Educational Research, 90(6), 375-380.

Andren, K. J. (2010). An analysis of the concurrent and predictive validity of curriculum based measures (CBM), the Measures of Academic Progress (MAP), and the New England Common Assessment Program (NECAP) for reading (Doctoral dissertation, The University of Southern Maine).

Bandura, A. (1977). Social Learning Theory. Englewood Cliffs, NJ: Prentice-Hall.

Barnette-Clarke, C., Fisher, W., Marks, R., Ross, S., \& Zbiek, R. M. (2010).

Developing Essential Understanding of Rational Numbers for Teaching Mathematics in Grades 3-5. Essential Understandings. Reston, VA: National Council of Teachers of Mathematics.

Battista, M.T. (2012) Cognition-Based Assessment and Teaching of Fractions: Building on Students' Reasoning. Portsmouth, N.H. Heinemann.

Brown, G., \& Quinn, R. J. (January 01, 2007). Investigating the relationship between fraction proficiency and success in algebra. Australian Mathematics Teacher, The, $63,4,8-15$.

Buffum, A., Mattos, M., \& Weber, C. (2009). Pyramid response to intervention. Bloomington, IN: Solution Tree Press.

Carbaugh, E. (2014, March 1). Designing reliable and valid common-core aligned math assessments. Using Assessments Thoughtfully. ASCD Express online publication, 9(12). http://www.ascd.org/ascd-express/vol9/912-carbaugh.aspx 
Chromebook [apparatus] (n.d.). Google.

Chval, K., Lannin, J., Jones, D., \& Dougherty, B. (2013). Putting essential understanding of fractions into practice. Reston, VA: National Council of Teachers of Mathematics.

Coleman, J. S., United States, \& National Center for Education Statistics. (1966). Equality of educational opportunity. Washington: U.S. Dept. of Health, Education, and Welfare, Office of Education; [for sale by the Superintendent of Documents, U.S. Govt. Print. Off.

Confrey, J., Nguyen, K. H., Lee, K., Panorkou, N., Corley, A. K., and Maloney, A. P. (2012). Turn-On Common Core Math: Learning Trajectories for the Common Core State Standards for Mathematics. URL: www.turnonccmath.net.

Confrey, J., Maloney, A. P., \& Corley, A. K. (2014). Learning trajectories: a framework for connecting standards with curriculum. ZDM, 46(5), 719-733.

Cronbach, L. J. (1951). Coefficient alpha and the internal structure of tests.psychometrika, 16(3), 297-334.

Data Management, Planning, and Program Evaluation (2015). [District data on assessed fractions skills]. Unpublished raw data.

Data Recognition Corporation (2007). Terra Nova. 
Dunham, P., \& Hennessy, S. (2008). Equity and use of educational technology in mathematics. In M.K. Heid \& G.W. Blume (Eds.) Research on technology and the teaching and learning of mathematics: Volume 1: research syntheses: A volume in research on technology and the teaching and learning of mathematics: syntheses, cases, and perspectives. (Vol. 1. Pp. 345-418) Charlotte, NC: Information Age Publishing, Inc.

Empson, S.B. (2001) Equal Sharing and the Roots of Fraction Equivalence. Teaching Children Mathematics 7 (March): 421-25.

Field, A. (2009). Discovering statistics using SPSS. Sage publications.

Fosnot \& Dolk. (2002) Young Mathematicians at Work: Constructing Fractions, Decimals, and Percent's. Portsmouth, N.H. Heinemann.

Gano, L. R. (2011). Fitting technology to the mathematics pedagogy: its effect on students' academic achievement. Journal of College Teaching \& Learning, 8(11), 29-38. Retrieved from http://search.proquest.com/docview/964185228?accountid=14665

Gersten, R. M., \& Newman-Gonchar, R. (2011). Understanding RTI in mathematics: Proven methods and applications. Los Alamitos, Ca: Instructional Research Group.

GoClass [Application] (2011). New York, NY: Learningmate Solutions. www.goclass.com

G*Power (2009) [Computer Software]. Düsseldorf, Germany.

Heddens, J. W. (1964). Today's mathematics: A guide to concepts and methods in elementary school mathematics. Chicago: S.R.A. 
Hennessy, S. (2006). Integrating technology into the teaching and learning of school science: A situated perspective on pedagogical issues in research. Studies in Science Education, 42(1), 1-48.

Herrera, T., Kanold, T.D., Koss, R.K., Ryan, P., \& Speer, W.R. (2007). Mathematics teaching today: Improving practice, improving student learning (2nd ed.). Reston, VA: National Council of Teachers of Mathematics.

Hill, H., Rowan, B., \& Ball, D. (2005). Effects of teachers' mathematical knowledge for teaching on student achievement American Educational Research Journal Summer, 42, (2), 371-406.

Holmes, K. (2009). Planning to teach with digital tools: Introducing the interactive whiteboard to pre-service secondary mathematics teachers. Australian Journal of Educational Technology, 25(3), 351-365.

Houghton Mifflin Harcourt (n.d.). GoMath: A research-based framework for Houghton Mifflin Harcourt GO Math! Grades K-6. Author.

Houghton Mifflin (2011). GoMath: Common Core Edition.

Hunter, J. E., \& Schmidt, F. L. (1990). Methods of meta-analysis: Correcting error and bias in research findings. Newbury Park: Sage Publications.

International Society of Technology Education. (2007). National Educational Technology Standards for Students. Author. Retrieved from: http://www.iste.org/standards/nets-for-students.

iPad [Apparatus and software]. (2014). Cupertino, CA: Apple Inc. 
Irving, K., \& Bell, R. (2004). Educational technology in standards and assessments for science and mathematics. Journal of Science Education and Technology, 13(2), 255- 266.

iTouch [Apparatus and software]. (2014). Cupertino, CA: Apple Inc.

Jonassen, D. (2000). Computers in the classroom: mindtools for critical thinking (2nd ed.). Englewood Cliffs, New Jersey: Merrill.

Kamii, C., \& Clark, F. B. (1995). Equivalent fractions: Their difficulty and educational implications. Journal of Mathematical Behavior, 14, 4, 365-378.

Karp, K. S., Bush, S. B., \& Dougherty, B. J. (2014). 13 Rules That Expire. Teaching Children Mathematics, 21, 1, 18-25.

Kastberg, S. E., \& Frye, R. S. (2013). Norms and Mathematical Proficiency. Teaching Children Mathematics, 20, 1, 28.

Keller-Margulis, M. A., Shapiro, E. S., \& Hintze, J. M. (2008). Long-Term Diagnostic Accuracy of Curriculum-Based Measures in Reading and Mathematics. School Psychology Review, 37, 3, 374-390.

Kelley, B., Hosp, J., \& Howell, K. (2008). Curriculum-Based Evaluation and Math. Assessment for Effective Intervention, 33, 4, 250-256.

Kennewell, S., Tanner, H., Jones, S., \& Beauchamp, G. (2008). Analysing the use of interactive technology to implement interactive teaching. Journal of Computer Assisted Learning, 24(1), 61-73.

Kent, P. (2006). Using interactive Whiteboards to Enhance Mathematics Teaching. [Electronic Version]. Australian Primary Mathematics Classroom, vol. 11 no. $2,23-26$. 
Kidspiration (Version 3.0) [Computer software]. Beaverton, OR: Inspiration Software, Inc.

Kilpatrick, J., Swafford, J., \& Findell, B. (Eds.). (2001). Adding it up: helping children to learn mathematics. Washington, DC: National Academy Press.

Koehler, M. J. \& Mishra, P. (2008). Introducing TPCK. In AACTE Committee on Innovation and Technology (Ed.), Handbook of Technological Pedagogical Content Knowledge (TPCK) for educators (pp. 3-29). New York: Routledge.

Mack, N. K. (1995). Confounding Whole-Number and Fraction Concepts When Building on Informal Knowledge. Journal For Research In Mathematics Education, 26(5), 422-41.

Manzo, K.K. (2010, Winter). Beyond Teacher Chalk Talk. Digital Directions, 3437.

Marzano, R. J., Haystead, M. W., \& Marzano Research Laboratory. (2009). Evaluation Study of the Effects of Promethean ActivClassroom on Student Achievement. Final Report. Marzano Research Laboratory. 555 North Morton Street, Bloomington, IN 47404. Tel: 888-849-0851; Fax: 866-801-1447; email: info@marzanoresearch.com; Web site: http://www.marzanoresearch.com.

Marzano, R. J., Pickering, D., \& Pollock, J. E. (2001). Classroom instruction that works: Research-based strategies for increasing student achievement. Alexandria, VA: Association for Supervision and Curriculum Development. McNamara, J. \& Shaughnessy, M.M. (2010). Beyond pizzas \& pies: 10 essential strategies for supporting fraction sense, grades 3-5. CA: Math Solutions. 
Means, B. \& Olson, K. (1994, April). The Link Between Technology and Authentic Learning. Educational Leadership, v51 n7 p15-18. Retrieved September 29 ${ }^{\text {th }}$, 2010, from EBSCOhost Academic Search Premier database.

Mishra, P., \& Koehler, M. (2006). Technological pedagogical content knowledge: A framework for teacher knowledge. The Teachers College Record,108(6), 1017-1054.

Morphew, V.N. (2012). A constructivist approach to the National Educational Technology Standards for Teachers. Eugene, OR: International Society For Technology in Education.

National Assessment of Educational Progress ((NAEP), 2007).

National Council of Teachers of Mathematics. (2000). Principles and standards for school mathematics. Reston, VA: Author.

National Council of Teachers of Mathematics. (2007). The Learning of Mathematics: 69th NCTM Yearbook [2007 NCTM Yearbook (69th)]. Reston, VA: National Council of Teachers of Mathematics.

National Council of Teachers of Mathematics. (2008). Mathematics teaching today: Improving practice, improving student learning (2nd ed.). Reston, VA: Author

National Governors Association Center for Best Practices, Council of Chief State School Officers ((NGA Center) 2010). Common Core State Standards.

Washington, D.C.: Authors. Retrieved from: http://www.corestandards.org Nexus 7 [Apparatus]. (2013). Google. 
Niess, M. L., Ronau, R. N., Shafer, K. G., Driskell, S. O., Harper, S. R., Johnston, C., Browning, C., Kersaint, G. (2009). Mathematics Teacher TPACK Standards and Development Model. Contemporary Issues in Technology and Teacher Education, 9, 1, 2009-3.

Northwest Evaluation Association (2003). Measures of Academic Progress. Portland, Oregon.

Oxford Desk Dictionary and Thesaurus (1997).

Pearson Assessment (2010).Group Mathematics Assessment and Diagnostic Evaluation.

Pedagogy (2014). In Merriam-Webster online dictionary. Retrieved from http://www.merriam-webster.com/dictionary/pedagogy

Petit, M.M, Laird, R.E., \& Marsden, E.L. (2010). A focus on fractions: bringing research to the classroom. New York: Routledge.

Petit, M.M, Laird, R.E., Marsden, E.L., \& Ebby, C.B. (2015). A focus on fractions: bringing research to the classroom: Second Edition. New York: Routledge.. Piaget, J. (1954). The construction of reality in the child. New York: Basic Books. Piburn, \& Sawada (2000). Reformed Teaching Observation Protocol (RTOP): Reference Manual (Report No. IN00-3). Arizona Collaborative for Excellence in the Preparation of Teachers.

Reinhart, J.M., Thomas, E.T., \& Toriskie, J.M. (2011) K-12 teachers: technology use and the second digital divide. Journal of Instructional Psychology, Vol. 38, No. 3, 181-193. 
Rittle-Johnson, B., Siegler, R. S., \& Alibali, M. W. (2001). Developing Conceptual Understanding and Procedural Skill in Mathematics: An Iterative Process. Journal of Educational Psychology, 93, 2, 346-62.

Rosenthal, R. (1984). Meta-analytic procedures for social research. Beverly Hills: Sage Publications.

Sawada, D., Piburn, M. D., Judson, E., Turley, J., Falconer, K., Benford, R., \& Bloom, I. (2002). Measuring reform practices in science and mathematics classrooms: The reformed teaching observation protocol. School Science and Mathematics, 102(6), 245-253.

Schmidt, W., Houang, R., \& Cogan, L. (2002). A Coherent Curriculum: The Case of Mathematics. American Educator, 26, 2, 10.

Shadish, W. R., Cook, T. D., \& Campbell, D. T. (2001). Experimental and quasiexperimental designs for generalized causal inference. Boston: Houghton Mifflin.

Shaughnessy. M. M. (2011) Identify Fractions and Decimals on a Number Line”. Teaching Children Mathematics 13 (March): 428-34.

Shavelson, R. J. (1996). Statistical reasoning for the behavioral sciences. Boston, Mass: Allyn and Bacon.

Shulman, L. (1986). Those who understand: Knowledge growth in teaching. Educational Researcher, 15(2), 4-14. Retrieved from http://www.jstor.org/stable/1175860 
Shulman, L. D. (1987). Knowledge and teaching: Foundations of the new reform. Harvard Educational Review, 57, 1-22.

Simon, M. A. (1995). Reconstructing mathematics pedagogy from a constructivist perspective. Journal for research in mathematics education, 114-145.

Staub, F., \& Stern, E. (2002). The nature of teacher's pedagogical content beliefs matters for students' achievement gains: Quasi-experimental evidence from elementary mathematics, Journal of Educational Psychology, 94(2), 344-355.

Strong, R., Silver, H., \& Perini, M. (2001). Teaching what matters most: standards and strategies for raising student achievement. Alexandria, VA: Association For Supervision and Curriculum Development.

Teddlie, C. \& Tashakkori, A. (2009). Foundations of mixed methods research: Integrating quantitative and qualitative approaches in the social and behavioral sciences. Thousand Oaks, CA: Sage.

Toumasis, C. (2004). Cooperative study teams in mathematics classrooms. International Journal of Mathematics Education and Science Technology, 35(5), 669-679.

United States. (2008). The National Mathematics Advisory Panel report: Foundations for success: hearing before the Committee on Education and Labor, U.S. House of Representatives, One Hundred Tenth Congress, second session, hearing held in Washington, DC, May 21, 2008. Washington: U.S. G.P.O. University of Michigan of Education (2015). High Leverage Practices. Retrieved from University of Michigan Teaching Works website: http://www.teachingworks.org/work-of-teaching/high-leverage-practices 
Van de Walle, J.A., (2007). Elementary and Middle School Mathematics: Teaching Developmentally Sixth Edition. Pearson Education, Inc.

Van de Walle, J.A., Karp, K.S., Bay-Williams, J.M., (2013). Elementary and Middle School Mathematics: Teaching Developmentally Eighth Edition. Pearson College Division.

Vygotsky̆, L. S., \& Cole, M. (1978). Mind in society: The development of higher psychological processes. Cambridge, MA: Harvard University Press.

Witzel, B. S. (2005). Using CRA to teach algebra to students with math difficulties in inclusive settings. Learning Disabilities-A Contemporary Journal,3(2), 4960. 


\section{APPENDIX A}

\section{Parental Informed Consent Letter}

February 17, 2015

Dear Parents/Guardians: University of Louisville on a research study by allowing investigators to collect testing results through
normal educational practice. There are no known risks for your participation in this research study. The
information collected may not benefit you directly. The information learned in this study may be helpful
to others. The assessment data collected will be used to determine the impacts of iPad technology on
student learning of fractions. Student data will have no identifying factors and will be saved in a
password protected excel file. Assessment data will be collected over the next ten weeks.

Individuals from the Department of Early Childhood and Elementary Education, the Institutional Review Board (IRB), the Human Subjects Protection Program Office (HSPPO), and other regulatory agencies may inspect these records. In all other respects, however, the data will be held in confidence to the extent permitted by law. Should the data be published, your identity will not be disclosed.

This study is taking part within the normal school day. Data is collected through normal school and instructional operations. Students will participate normal classroom procedures, including testing procedures. You may request that your students' tests scores not be collected for the purpose of this study. If you do not wish for your students' scores to be collected for the purpose of this study you must sign the waiver form attached to this document. You may keep this letter for your records. The information we collect from the education or study records of you or your student may only be used to meet the purposes of the study as stated in this consent. If you have anv auestions, concerns, or complaints about the research study, please contact: Shannon Stone at

If you have any questions about your rights as a research subject, you may call the Human Subjects Protection Program Office at (502) 852-5188. You can discuss any questions about your rights as a research subject, in private, with a member of the Institutional Review Board (IRB). You may also call this number if you have other questions about the research, and you cannot reach the research staff, or want to talk to someone else. The IRB is an independent committee made up of people from the University community, staff of the institutions, as well as people from the community not connected with these institutions. The IRB has reviewed this research study.

If you have concerns or complaints about the research or research staff and you do not wish to give your name, you may call $1-877-852-1167$. This is a 24 hour hot line answered by people who do not work at the University of Louisville.

Sincerely,

Shannon Stone, Ph.D Canidate
Karen Karp, Ph.D 


\title{
APPENDIX B
}

Parent Waiver Letter

\begin{abstract}
THE IMPACT OF INTERACTIVE TECHNOLOGY ON ELEMENTARY STUDENT ACHIEVEMENT IN FRACTIONS
\end{abstract}

\author{
Subject Informed Waiver
}

\section{THE IMPACT OF INTERACTIVE TECHNOLOGY ON ELEMENTARY STUDENT ACHIEVEMENT IN FRACTIONS}

Investigator(s) name $\&$ address:

Sponsor: Karen Karp, Ph.D.

Department of Early Childhood and Elementary Education

Room 243 - College of Education and Human Development

\section{Shannon Stone}

Site(s) where study is to be conducted: Phone number for subjects to call for $\mathrm{q}$

\section{Introduction and Background Information}

A research study is being conducted within the normal classroom day. The study is being conducted by Shannon Stone who is a PhD candidate at the University of Louisville. The study is sponsored by Karen Karp, Ph.D. Department of Early Childhood and Elementary Education Room 243 - College of Education and Human Development at the University of Louisville. The study will take place at Kerrick Elementary and Shacklette Elementary. Approximately 368 subjects will be invited to participate.

\section{Purpose}

Significant gains in student achievement are reported through the use of interactive technology. These gains are reported due to the level of engagement students experiences while interacting with technology. Technology is a quickly changing field. Importance should be placed on how various types of technology can impact student learning and achievement. The purpose of this study is to explore the impact of interactive technology on fraction proficiency.

\section{Voluntary Participation}

This study is taking part within the normal school day. Students will participate normal classroom procedures, including testing procedures. Participation in normal classroom procedures is not voluntary. Data is collected through normal school assessment procedures. You may request that your students' tests scores not be collected for the purpose of this study. The information we collect from the education or study records of you or your student may only be used to meet the purposes of the study as stated in this consent. We will conduct this study in a manner that does not allow identification of you or your child by anyone other than study team members or others who may have a legitimate reason to know. All

Page 1 of 3 


\section{THE IMPACT OF INTERACTIVE TECHNOLOGY ON ELEMENTARY STUDENT ACHIEVEMENT IN FRACTIONS}

instructional materials or survey instruments used for the research, including teachers' manuals, films, tapes, or other supplementary instructional material used in connection with this study, are available for you to see before the study begins if you ask to see it. If you want to see any of this information, please contact Shannon Stone or Karen Karp at $5 \quad 84$ and they will give you a date and time where it will be available for you to review. Once we have completed this study, we are required by the U.S. Department of Education to destroy or return to the school system all personally identifiable information when no longer needed for the purposes of the study. We expect this study to last for three months and we will destroy or return the information to the school system by May 30,2015.

Research Subject's Rights, Questions, Concerns, and Complaints

If you have any concerns or complaints about the study or the study staff, you have three options.

You may contact the principal investigator at $5 0 \longdiv { 4 }$

If you have any questions about your rights as a study subject, questions, concerns or complaints, you may call the Human Subjects Protection Program Office (HSPPO) (502) 852-5188. You may discuss any questions about your rights as a subject, in secret, with a member of the Institutional Review Board (IRB) or the HSPPO staff. The IRB is an independent committee composed of members of the University community, staff of the institutions, as well as lay members of the community not connected with these institutions. The IRB has reviewed this study.

If you want to speak to a person outside the University, you may call 1-877-852-1167. You will be given the chance to talk about any questions, concerns or complaints in secret. This is a 24-hour hot line answered by people who do not work at the University of Louisville.

This paper tells you what will happen during the study. Your signature means that you wish to have your students test scores withdrawn from the study. By not returning this form, investigators will include your students' assessment scores in the data collection procedures. This informed consent document is not a contract. You are not giving up any legal rights by signing this informed consent document.

Signature of Subject/Legal Representative Date Signed

Signature of Investigator

Date Signed

LIST OF INVESTIGATORS

PHONE NUMBERS

Karen Karp, Ph.D.

Shannon Stone, Ph.D. Candidate

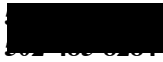

Page 2 of 3 
THE IMPACT OF INTERACTIVE TECHNOLOGY ON ELEMENTARY STUDENT ACHIEVEMENT IN FRACTIONS

Page 3 of 3 


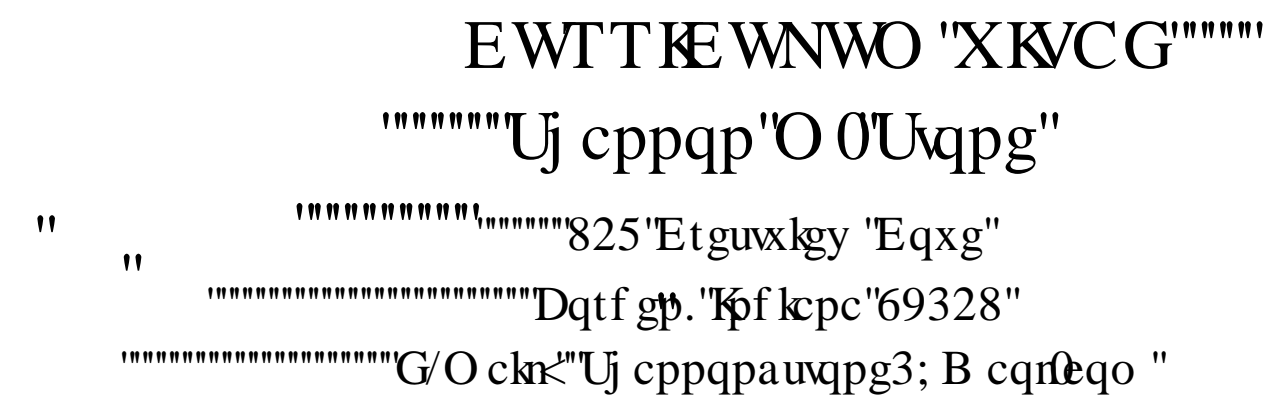

\title{
EDUCATION
}

Ph.D. 2015

\author{
University of Louisville, Louisville, Kentucky \\ Concentration: Elementary Mathematics and Instructional \\ Technology \\ Dissertation Chair: Dr. Karen Karp \\ Dissertation Title: THE IMPACT OF AN INTERACTIVE \\ TECHNOLOGY APPLICATION ON STUDENT \\ ACHIVEMENTIN FRACTIONS.
}

Rank I 2008

University of Louisville, Louisville, Kentucky

Elementary Education and Reading Instruction

M.A. 2005

Spalding University, Louisville, Kentucky

Master of Arts in Teaching

Concentration: Learning and Behavior Disorders

B.A. 2003 University of Kentucky, Lexington, Kentucky

Bachelor of Arts in Sociology

Minor: Psychology

PROFESSIONAL CERTIFICATION

- Consultant Exceptional Children

- Consultant Elementary Education

- Learning and Behavior Disorders P-12

- Elementary Education P-5

- Kentucky Reading Project Endorsed 


\section{PROFESSIONAL EXPERIENCE}

August 2014 - Current

August 2008-August 2014

August 2004-August 2008

\section{Student Response Team Coach, Jefferson County Public Schools, Louisville Kentucky}

Assist administrative staff in discipline issues. CoDFK teachers in Positive Behavior Intervention Supports in the classroom. Execute small group social skills sessions and skill streaming for targeted behaviors. Work closely with family resource coordinator, school social workers, and consulting teachers to help remove barriers to student education.

\section{Teacher, Kerrick Elementary, Louisville, Kentucky} Instruct intermediate grades common core state Math and English Language Arts standards, design and develop programs to meet the academic, intellectual, and social needs of all students. Instruct intense fiveblock programs in literacy, Math Investigations II , inquiry based science, and social studies. Assess student performance formatively to drive instruction. Keep accurate intervention records.

\section{LBD Teacher, Kerrick Elementary, Louisville, Kentucky}

Instruct emotional and behavior disorder students through the design and development of programs to meet their academic, intellectual, and social needs. Instruct intense five-block programs in literacy, inquiry based science, social studies, and mathematics. Assess student performance formatively to provide tailored instruction. Progress monitor goals, and ensure students are receiving appropriate supplementary aides, accommodations, and services. Meet with Site-Based Admissions and Release Committees, Collect data and develop Individual Education Plans for students with disabilities. Work with outside agencies to ensure students' social and emotional needs are being met. Work with district personnel to develop a functioning EBD unit. 


\section{UNIVERSITY TEACHING}

EDAP 611 Lead Technology Instructor, Summer Portfolio Institute. University of Louisville

EDTP 313/604 Co-Instructor, Elementary Mathematics Methods. University of Louisville

\section{CONSULTING EXPERIENCE}

Product consultant (2014-2015) for LearningMate Solutions for the International Society of Technology Education Annual Meeting.

Product consultant (2014) for LearningMate Solutions for the Florida Education Technology Exposition Annual Meeting.

\section{OTHER PROFESSIONAL EXPERIENCE}

Featured Teacher (2012) for integrating tablet technology in instruction for a feature on the GoClass application on The Leading Edge Series.

Master Teacher (2011-2012) for School Improvement Network: PD 360 Professional Development Series.

\section{PEER REVIEWED PUBLICATIONS}

Brown, E.T, Bush, S., \& Washburn, S. (April 2012). Technology, Translations and Treasure Hunts. Middle Ground Magazine. April 2012. Pgs. 20-21.

\section{PEER REVIEWED PRESENTATIONS}

Stone, S. (January, 2016). Flipping Elementary ELA Instruction the Easy Way! Florida Education Technology Convention, Orlando, Florida.

Stone, S. (January, 2016). The Impacts of Interactive Technology on Elementary Fractions Learning. Florida Education Convention, Orlando, Florida.

Stone, S. (November, 2014). Primary Number and Operations Through an Interactive Technology Lens. Presentation at the National Council of Mathematics Regional Conference, Richmond, Virginia. 
White (Dix), Leah \& Stone, S. (March, 2014). Mathematics and Interactive Technology. Kentucky Council of Mathematics Spring Conference, Lexington, Kentucky.

Stone, S. (March, 2014). Learning Your Students Will Flip Over. Presentation at Kentucky Society of Technology Educators Spring Conference, Louisville, Kentucky.

Dix, Leah \& Stone, S. (November, 2013). Applying Interactive Technology To Mathematics. Presentation at the National Council Of Mathematics Regional Conference, Louisville, Kentucky.

Dix, Leah \& Stone, S. (October, 2013). Applying Interactive Technology To Mathematics. Presentation at the National Council Of Mathematics Regional Conference, Las Vegas, Nevada.

Stone (Washburn), S. (March, 2012). Responding to Struggling Learners through Professional Learning Communities. Poster Presentation at University of Louisville's Spring Research Conference, Louisville, KY.

\section{INVITED PRESENTATIONS}

Stone, S. (July, 2014). Learning Your Students Will Flip Over. Professional Development Presentation Given at Jefferson County Public Schools Technoversity, Louisville, KY.

Stone (Washburn), S. (July, 2012). Using Technology to Build a Classroom Community. Professional Development Presentation given at Jefferson County Public Schools Technoversity, Louisville, KY.

Stone (Washburn), S. (July 2012). SMART Board and English Language Arts Common Core Standards. Professional Development Presentation given at Jefferson County Public Schools Technoversity, Louisville, KY.

\section{HONORS AND AWARDS}

Dean's Citation Award (2015). University of Louisville Doctoral Hooding Ceremony.

\section{LEADERSHIP EXPERIENCE}

2008-present Staff Enhancement and Building Relations Committee Leader

2011-2013 Regional Director, Jefferson County Teachers Association

2007-2011 Co-Sponsor Nature Club

2005-2010 Building Science Teacher Leader

2005-2010 School-wide Instructional Leadership Team

2007-2008 Site-Based Decision Making Team 
2006-2008 Jefferson County Public Schools Hiring Committees for staffing Stopher Elementary, and Farmer Elementary Schools. Teachers and Learners in Collaboration for Semple Elementary School. 